US Army Corps

of Engineers ${ }_{\circledast}$

Engineer Research and

Development Center

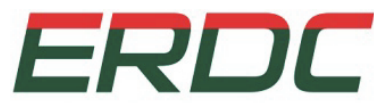

INNOVATIVE SOLUTIONS

for a safer, better world

\title{
Summary Review of the Aquatic Toxicology of Munitions Constituents
}

Guilherme R. Lotufo, Gunther Rosen, William Wild,

June 2013 and Geoffrey Carton

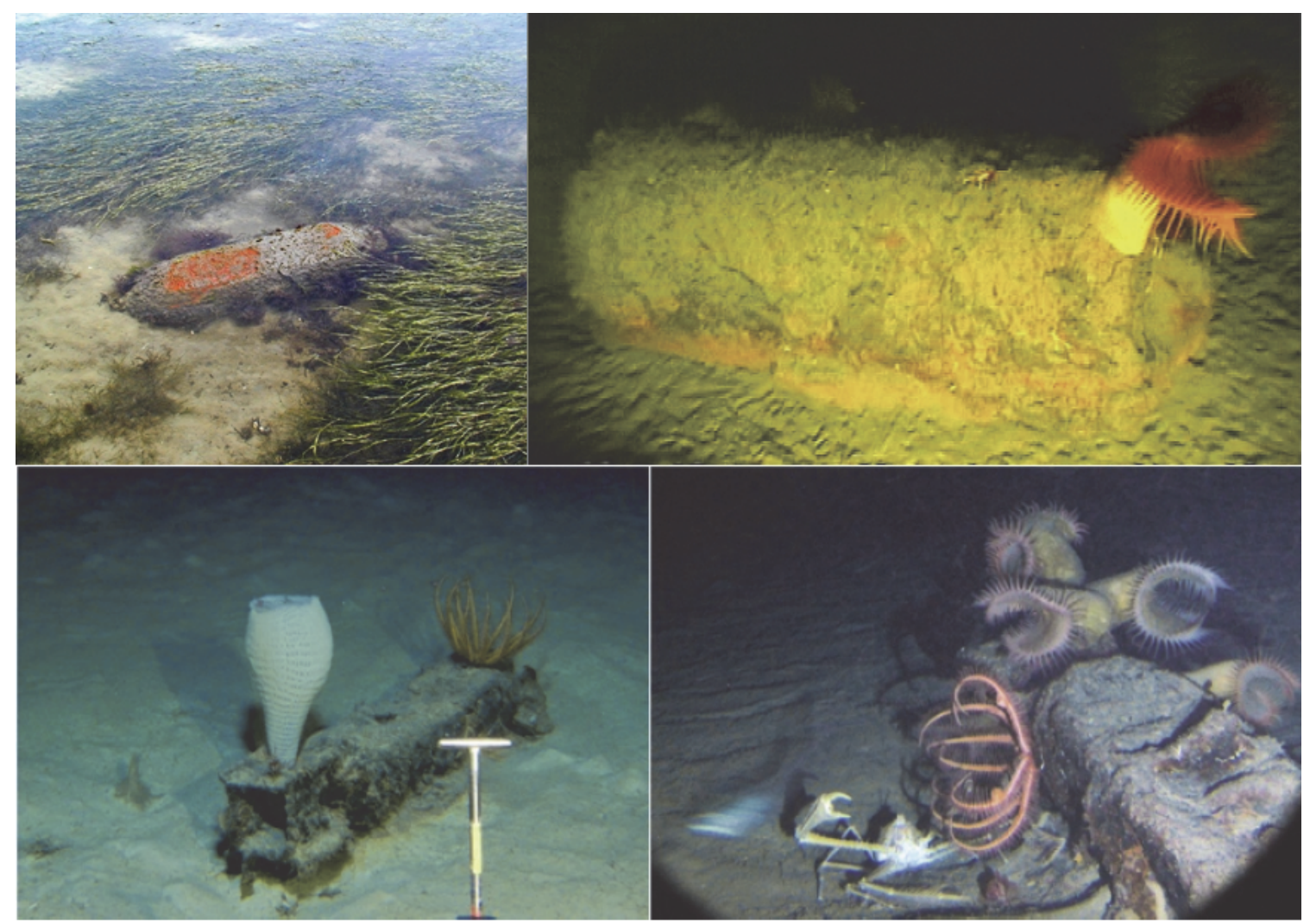


The US Army Engineer Research and Development Center (ERDC) solves the nation's toughest engineering and environmental challenges. ERDC develops innovative solutions in civil and military engineering, geospatial sciences, water resources, and environmental sciences for the Army, the Department of Defense, civilian agencies, and our nation's public good. Find out more at www.erdc.usace.army.mil.

To search for other technical reports published by ERDC, visit the ERDC online library at http://acwc.sdp.sirsi.net/client/default. 


\title{
Summary Review of the Aquatic Toxicology of Munitions Constituents
}

\author{
Guilherme R. Lotufo \\ Environmental Laboratory \\ U.S. Army Engineer Research and Development Center \\ 3909 Halls Ferry Road \\ Vicksburg, MS 39180 \\ Gunther Rosen and William Wild \\ Space and Naval Warfare Systems Center Pacific \\ 53560 Hull Street \\ San Diego, CA 92152-5001 \\ Geoffrey Carton \\ Office of the Deputy Assistant Secretary of the Army \\ for Environment, Safety and Occupational Health \\ Support Contractor/CALIBRE Systems \\ 6354 Walker Lane \\ Alexandria, VA 22310
}

Final report

Approved for public release; distribution is unlimited.

\author{
Prepared for U.S. Army Corps of Engineers \\ Washington, DC 20314-1000 \\ Under Work Unit 33143 \\ Monitored by U.S. Army Engineer Research and Development Center \\ 3909 Halls Ferry Road, Vicksburg, MS 39180-6199
}




\section{Abstract}

Military munitions are present in waters around the world, including those waters located at current and former Department of Defense sites. This report provides a review of the aquatic ecotoxicology of munitions constituents (MC), including nitroaromatics (2,4,6-trinitrotoluene (TNT), dinitrotoluenes (DNTs), 1,3,5-trinitrobenzene (TNB), 2,4,6trinitrophenylmethylnitramine (tetryl) and 2,4,6-trinitrophenol (picric acid)); nitrate esters (nitrocellulose (NC), pentaerythritoltetranitrate (PETN), nitroglycerine (NG)); and nitramines (hexahydro-1,3,5- trinitro-1,3,5-triazine (RDX), and octahydro-1,3,5,7-tetranitro-1,3,5,7-tetrazocine (HMX)). The major focus of this report is on the fate and effects of $\mathrm{MC}$ in the marine environment. Most MC observed in this study rapidly degraded in aqueous exposure systems and nitroaromatics showed a significant binding affinity with organic matter. To support the assessment of risk from $\mathrm{MC}$ in aquatic environments, laboratory-based toxicity data have been derived for a variety of aquatic species for both lethal and sublethal exposure endpoints using spiked water or sediment. Frequently, unrealistically high concentrations were used to derive toxicity benchmarks. In general, nitramines were less toxic than nitroaromatics, with a wide range of sensitivity among species. MC are weakly hydrophobic and bioaccumulative potential was low, as expected. High elimination rates for $\mathrm{MC}$ resulted in a virtually complete loss of body residue within hours to days following transfer to clean water. Uptake of TNT resulted in the substantial formation of bound residues. For fish, aqueous exposure was the dominant route of exposure to explosive compounds, with dietary uptake providing only minimal contribution. More realistic exposures using Composition B and multiple species found the presence of munitions in aquatic environments unlikely to result in biological effects. Verification of this conclusion should be pursued by determining site-specific exposure risk.

DISCLAIMER: The contents of this report are not to be used for advertising, publication, or promotional purposes. Citation of trade names does not constitute an official endorsement or approval of the use of such commercial products. All product names and trademarks cited are the property of their respective owners. The findings of this report are not to be construed as an official Department of the Army position unless so designated by other authorized documents.

DESTROY THIS REPORT WHEN NO LONGER NEEDED. DO NOT RETURN IT TO THE ORIGINATOR. 


\section{Contents}

Abstract........................................................................................................................................... if

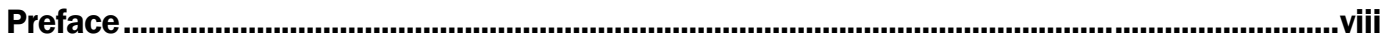

1 Introduction.......................................................................................................................... 1

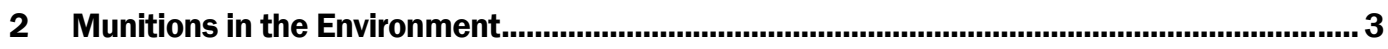

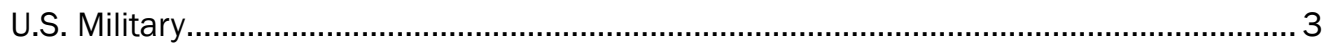

Underwater Munitions............................................................................................... 3

Munitions in the Marine Environment............................................................................ 4

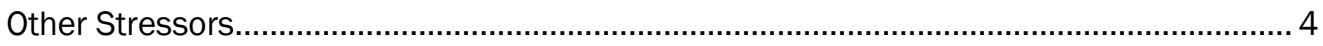

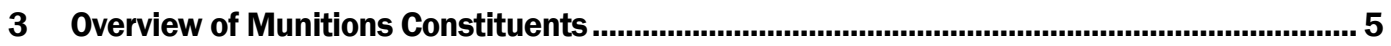

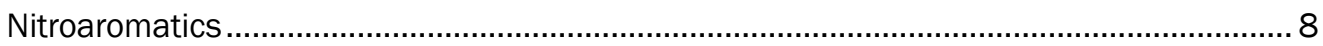

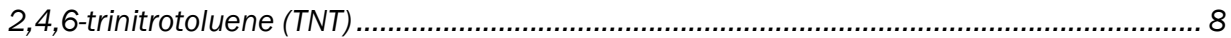

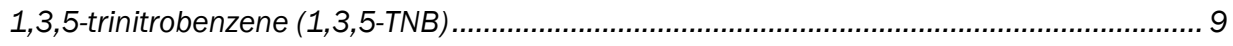

2,4-and 2,6- Dinitrotoluene (2,4-DNT and 2,6-DNT) ......................................................... 9

Tetryl, Ammonium Picrate (Explosive D), and Picric Acid..................................................... 10

Cyclyic Nitramines ...................................................................................................... 12

Other Energetic Compounds...................................................................................... 13

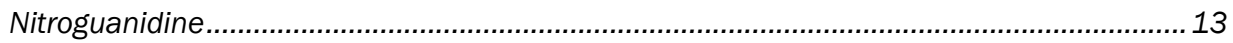

Nitrate Esters: nitroglycerin, nitrocellulose, PETN and DEGDN (diethylene glycol

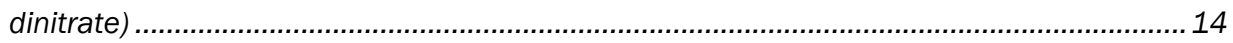

4 Aqueous Toxicity of Munitions Constituents to Fish and Aquatic Invertebrates..................15

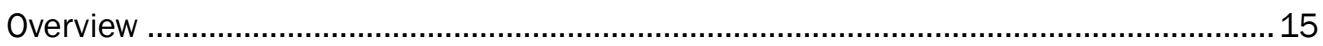

Fate of MC in exposure water during toxicity testing..................................................... 16

Toxicity of nitroaromatic MC................................................................................ 19

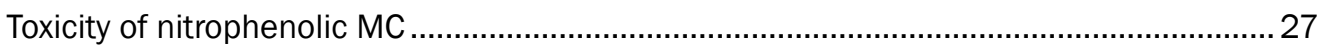

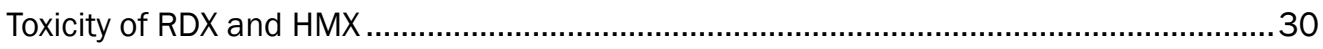

Toxicity of nitroguanadine, nitrogyclerin, nitrocellulose, PETN, and DEGDN.....................34

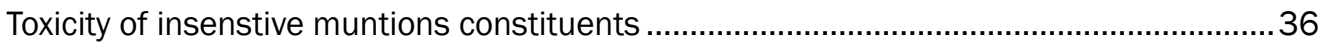

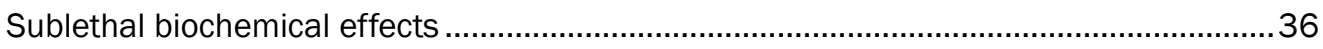

Relationship between bioaccumulation of MC and toxicity ............................................38

5 Toxicity of Munitions Constituents to Aquatic Invertebrates and Fish in Sediment Exposures.............................................................................................................................42

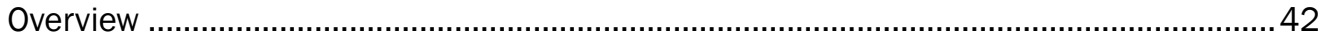

Fate of nitroaromatic MC amended to sediment for toxicity testing .................................43

Toxicity of nitroaromatic MC amended to sediment .....................................................49

Fate and toxicity of nitrophenolic MC in sediment .........................................................53

Toxicity and fate of nitramine MC in sediment ................................................................5

Sublethal toxicity and stimulatory effects of MC in sediment exposures ..........................57 
Toxicity of porewater extracted from sediments spiked with explosives ............................58

Preliminary sediment quality benchmarks ..................................................................59

6 Toxicokinetics and Bioconcentration of Munitions Constituents ......................................61

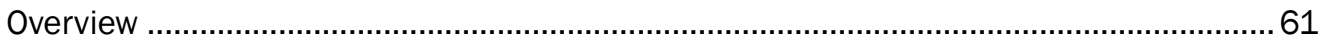

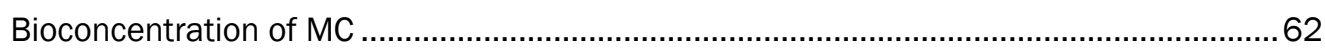

$\mathrm{MC}$ as bioccumulative compounds in a regulatory context ...........................................64

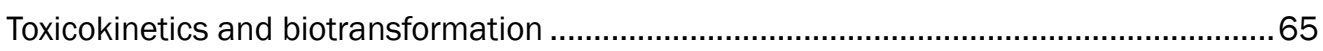

Formation and Elimination of TNT Bound Residues in Marine Invertebrates and

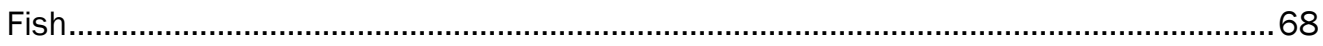

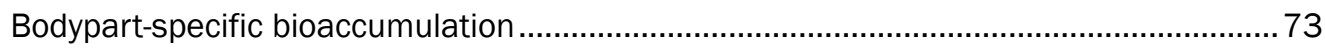

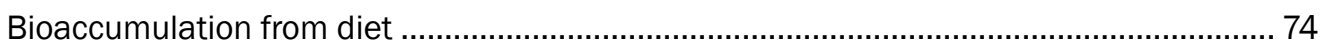

7 Fate, Toxicity, and Bioaccumulation of MC Released from Composition B under

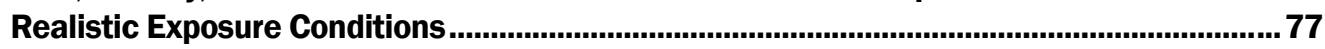

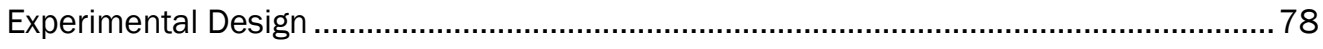

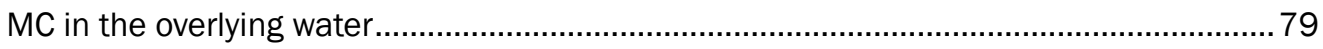

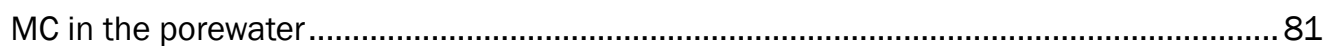

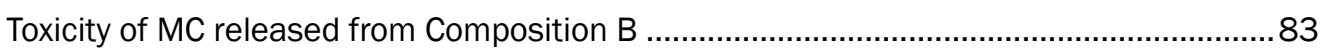

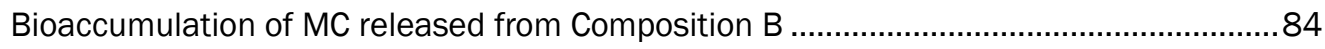

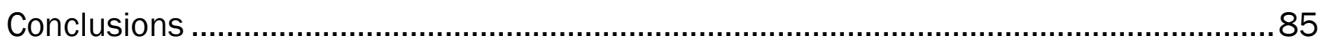

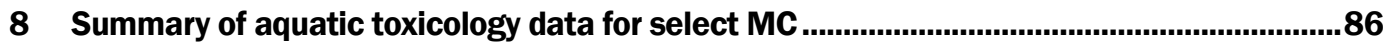

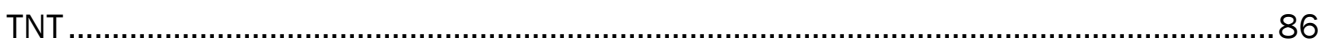

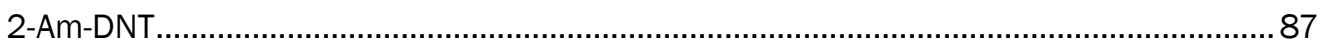

4-Am-DNT

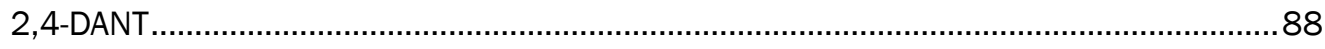

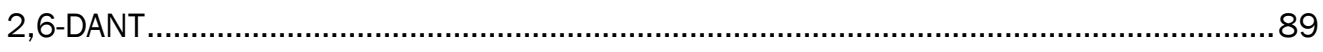

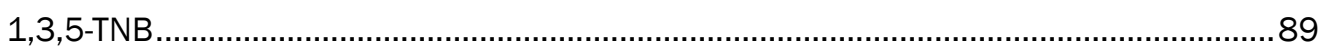

2,4-DNT

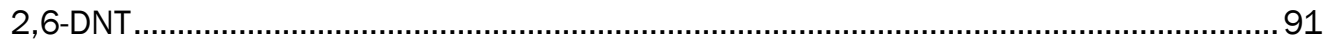

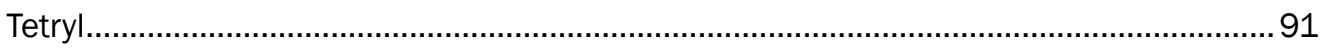

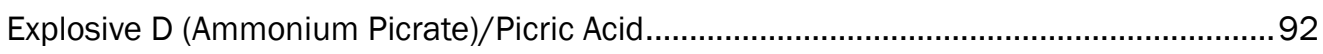

RDX

HMX

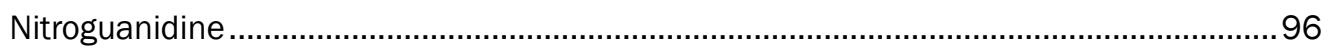

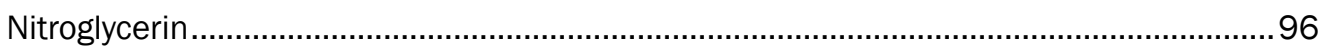

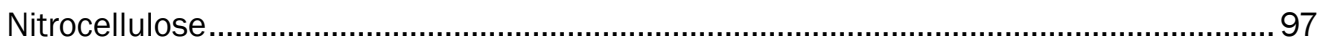

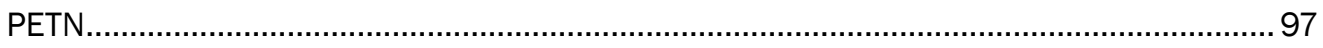

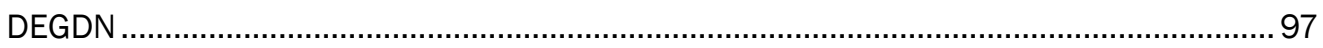

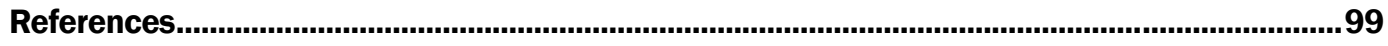

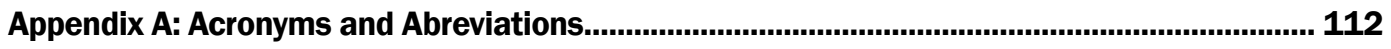

Report Documentation Page 


\section{Figures and Tables}

\section{Figures}

Figure 4.1. Temporal change in aqueous concentrations of SumTNT expressed as a percent of the initial concentration during the TNT 10-d static renewal experiment (left) and the TNT 10-d constant exposure experiment (right).

Figure 4.2. Mean percent $\mathrm{MC}$ lost in all test concentrations during aqueous exposures

Figure 4.3. Lethal toxicity data for marine fish and invertebrates exposed to TNT. LC50 median lethal concentration. See Table 4.1 for references.

Figure 4.4. Mean percent survival of juvenile sheepshead minnows (Cyprinodon variegatus) at termination of the 5-d exposure to aqueous exposures to TNT, 2-Am-DNT, 1,3,5-TNB and 2,4-DANT (on log scale) (from Lotufo et al. 2010a)

Figure 5.1. Percent of total concentration in sediment samples corresponding to the concentration of trinitrotoluene (TNT), 2-aminodinitrotoluene (2-Am-DNT), 4aminodinitrotoluene (4-Am-DNT), and diaminonitrotoluenes (DANTs) in solvent extracts of ${ }^{14} \mathrm{C}$-TNT-spiked Yaquina Bay (YB) and San Diego Bay (SD) toxicity test sediments..

Figure 5.2. TNT and transformation product concentrations over time in fine-grained marine sediment.

Figure 5.3. Percent of total radioactivity in sediment corresponding to the sum of solventextracted TNT and all its transformation products, including unknown compounds, as determined by radioactivity (blue), and to the sum of solvent-extracted TNT, Am-DNTs and DANTs, as determined by chemical analysis (red) in sandy sediment (Yaquina Bay, YB) and fine-grained sediment (San Diego Bay, SD) at initiation (Day 0) and termination (Day 10) of a toxicity test

Figure 5.4. Percentage of total radioactivity from radiolabeled-TNT in the exposure chamber that was associated with various media at toxicity test initiation (Day 0 ) and termination (Day 10) for sandy sediment (Yaquina Bay, YB) or fine-grained sediment.

Figure 5.5. Percentage of the total radioactivity from RDX or HMX in the exposure chamber that was associated with sediment and overlying water at termination of a toxicity test

Figure 5.6. Percentage of the total mass of RDX in the exposure chamber that was associated with various media at toxicity test termination for a sandy sediment

Figure 6.1. Bioconcentration factors (BCF) for freshwater fish and invertebrates exposed to TNT determined for body residues for parent compound, sum concentration of extractable compounds and for the sum concentration of extractable and non-extractable compounds.

Figure 6.2. Proposed metabolic activation and formation of protein-bound TNT residues in fish and aquatic invertebrates. The red box indicates the identification of Am-DNTs and DANTs as hydrolysable bound residues in tissue of TNT-exposed earthworms...

Figure 6.3. Depuration regressions for sheepshead minnows (left) or mussels (right) exposed to ${ }^{14} \mathrm{C}$-TNT-spiked seawater (Lotufo et al. unpublished study).

Figure 7.1. Exposure system used to assess fate and effects of Composition B

Figure 7.2. TNT concentration in the overlying water from exposures containing Composition B fragments under both static and flow conditions. 
Figure 7.3. The concentration of RDX in the overlying water from exposures containing Composition B fragments, under both static and flow conditions.

Figure 7.4. Mean ( \pm 1 standard deviation) SumTNT (left) and RDX (right) porewater concentrations at termination of exposures with Composition B fragments.

\section{Tables}

Table 3.1. Energetic chemicals present in military explosive and propellant formulations 6

Table 3.2. Physicochemical properties of munitions constituents addressed in this report.............. 7

Table 4.1. Lethal toxicity data for marine fish and invertebrates exposed to nitroaromatic MC................ 20

Table 4.2. Lethal toxicity data for freshwater fish, amphibians and invertebrates exposed to TNT.

Table 4.3. Lethal toxicity data for fish, a tadpole and aquatic invertebrates exposed to TNT,

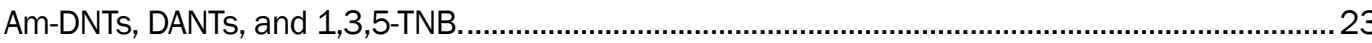

Table 4.4. Sublethal toxicity data for marine invertebrates and alga exposed to nitroaromatic MC, and comparison with lethal toxicity data, when available.

Table 4.5. Sublethal toxicity data for freshwater cladoceran Ceriodaphnia dubia exposed to nitroaromatic MC, and comparison with lethal toxicity data, when available.

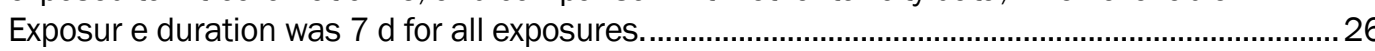

Table 4.6. Toxicity data for freshwater animals exposed to DNTs. ................................................... 27

Table 4.7. Lethal and sublethal effects concentrations for marine fish, invertebrates and

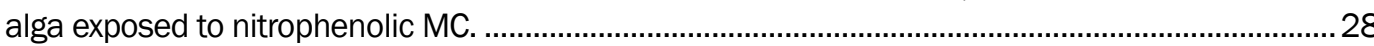

Table 4.8. Lethal concentrations for freshwater fish and invertebrates exposed to nitrophenolic MC.

Table 4.9. Lethal and sublethal toxicity data for marine fish and invertebrates exposed to RDX or HMX.

Table 4.10. Chronic toxicity data for fish and aquatic invertebrates exposed to RDX.......................32

Table 4.11. Toxicity data for freshwater fish and invertebrates exposed to HMX. ............................34

Table 4.12. Lethal and sublethal toxicity data for marine and freshwater fish and invertebrates exposed to propellant related energetic compounds.

Table 4.13. Body residue toxicity metrics for freshwater and marine invertebrates exposed to nitroaromatic MC

Table 5.1. Target concentrations and concentrations measured after spiking with TNT and mixing for sediments of different types and total organic carbon (TOC) content used in toxicity tests.

Table 5.2. Lethal toxicity data for nitroaromatic MC determined for aquatic invertebrates using spiked sediment exposures.

Table 5.3. Toxicity of nitrophenolic MC was determined for the marine amphipod Ampelisca abdita using spiked sediment exposures (Nipper et al. 2002).

Table 5.4. Toxicity of RDX and HMX determined for aquatic invertebrates using spiked sediment exposures.

Table 5.5. Toxicity of marine porewater extracted from sandy or fine-grained sediments spiked with 2,6-DNT, tetryl or picric acid (from Nipper et al. 2002).

Table 5.6. Marine sediment quality benchmarks (SQB) and no observed effect concentration (NOEC) from sediment toxicity tests for nine MC. 
Table 6.1. Empirically determined bioconcentration factor (BCF) values for fish and aquatic invertebrates.

Table 6.2. A comparison of predicted versus measured bioconcentration factor (BCF)

values.

Table 6.3. Toxicokinetic model parameters for fish and aquatic invertebrates exposed to TNT, 2-Am-DNT, 2,4-DANT, 4-DNT, RDX and HMX. See Table 5.2 for species habitat information. 66

Table 6.4. Bioconcentration factor (BCF) values for aquatic animals determined using the sum concentration of the parent compound and transformation products obtained from radioactivity in the extract or whole body as surrogate for body residue.....

Table 6.5. Percentage of total body residue for solvent extractable compounds in fish and mussels

Table 6.6. Percentage of total body residue for solvent-extracted compounds and for bound (non-extractable) residues in fish and mussels exposed to radiolabeled TNT.

Table 6.7. Mean elimination half-lives for SumTNT and for nonextractable residues in fish and mussels

Table 6.8. Contribution (as a percent) of different body parts to the total amount of bioaccumulated compounds for different classes of analytes in sheepshead minnows exposed to TNT, RDX and HMX

Table 7.1. Test organism survival, and embryo-larval development success following exposures with Composition $B$ fragments.

Table 7.2. Body burdens from Compositon B fragment exposures 


\section{Preface}

This summary review was conducted for the Office of the Assistant Secretary of the Army (Installations, Energy \& Environment) and the U.S. Navy's Environmental Sustainability Development to Integration Program.

The work was performed by Dr. Guilherme Lotufo of the Environmental Risk Assessment Branch (ERAB), Environmental Processes and Engineering Division (EPED), of the Environmental Laboratory (EL), U.S. Army Engineer Research and Development Center (ERDC). Gunther Rosen and William Wild of the Space and Naval Warfare Systems Center Pacific, and Geoffrey Carton, Support Contractor-CALIBRE Systems of the Office of the Deputy Assistant Secretary of the Army for Environment, Safety and Occupational Health. Technical reviews were provided by Jacob Stanley and Sandra Brasfield, ERAB, and Robert George from Space and Naval Warfare Systems Center Pacific, San Diego, CA.

This work was conducted under the general supervision of Buddy Goatcher, Chief, ERAB, and Warren Lorentz, Chief, EPED. At the time of publication of this report, Dr. Beth Fleming was Director of EL.

COL Kevin J. Wilson was Commander of ERDC and Dr. Jeffery Holland was Director. 


\section{Introduction}

As a result of military training and weapons testing activities, munitions are present at numerous current and former Department of Defense (DoD) sites. Many active and former military installations have ranges and training areas that include aquatic environments, such as ponds, lakes, rivers, estuaries and coastal ocean areas. In addition, until 1970, it was accepted practice to dispose of wastes - including excess, obsolete and unserviceable munitions - in deepwater areas. Munitions are also present in waters around the world due to combat and training operations.

Munitions constituents (MC) are defined in 10 U.S.C. 2710 (e) (4) as "Any materials originating from unexploded ordnance, discarded military munitions, or other military munitions, including explosive and nonexplosive materials, and emission, degradation, or breakdown elements of such ordnance or munitions." Additional definitions of terms used herein are provided in Appendix A. This report addresses the aquatic ecotoxicology of explosives, propellants, and related compounds, with a focus on the marine environment. This report does not address the aquatic ecotoxicology of other MC, such as insensitive munitions, perchlorate, white phosphorus, and metals released from both initiating primers and corrosion of munitions casings.

The following sections provide a review of the scientific literature concerning the investigation of the aquatic toxicity and bioaccumulation potential of MC parent compounds and transformation products in sediment and aqueous exposures. Those studies contain valuable information about the use in the assessment of ecological risks potentially associated with releases of these compounds into the aquatic environment. Each section summarizes the results of studies published in peer-reviewed literature as well as in a few unpublished studies conducted by the authors of this report. More detailed information associated with these studies can be obtained from the cited articles.

- Section 2 provides an overview of munitions found in the environment.

- Section 3 provides an overview of MC and some background information on the explosives and their degradation products addressed in this report. 
- Section 4 summarizes aqueous toxicity data for major explosive compounds and their transformation products to marine fish and marine invertebrates. The exposures used in the studies described in this section involved the addition of pure compounds to water at high enough concentrations to induce effects and calculate toxicity metrics (both aqueous and tissue-based) using standard laboratory methods.

- Section 5 summarizes sediment toxicity exposures of major explosive compounds to marine invertebrates. Toxicity exposures involved the addition of single compounds to sediment at high enough concentrations to induce effects and calculate toxicity metrics. This section provides information on the fate of MC during the storage and exposure period following spiking for several different sediment types, as well as the effects of sediment-associated MC to a variety of invertebrates and fish.

- Section 6 describes results from the bioaccumulation potential investigations of 2,4,6-trinitrotoluene (TNT) (and its major transformation products), Royal Demolition Explosive (RDX), and High Melting Explosive (HMX) in marine fish and invertebrates. Uptake rates, elimination rates, half lives, and bioconcentration factors (BCF) are reported. Body-part-specific bioaccumulation in marine fish following exposure to TNT, RDX, and HMX are also presented. Section 6 also provides information about the formation and elimination kinetics of nonextractable tissue-bound TNT and its metabolites in fish and mussels. A summary of body-part-specific bioconcentrations of MC and of the potential for dietary uptake of the explosive TNT to fish are also presented.

- Section 7 addresses the fate and effects of TNT and RDX released from Composition B fragments in microcosm exposures. These experiments involved a variety of endpoints for marine fish and invertebrates to characterize dissolution and potential biological exposure and effects under "realistic" scenarios using different exposure scenarios with multiple species.

- Section 8 summarizes marine toxicity and bioaccumulation data of MC for which research has been conducted.

- Section 9 provides the references cited in this report. 


\section{Munitions in the Environment}

Munitions in the environment may release explosive residuals due to corrosion and breaching in terrestrial and aquatic sites or due to blow-inplace (BIP) detonation (Pennington et al. 2008; Lewis et al. 2009; Pascoe et al. 2010). Munitions also pose risks associated with the physical impacts of accidental detonation (MacDonald et al. 2009). Areas where explosives impact soil or sediment quality are sometimes extensive; some artillery ranges are several square miles in area. The $\mathrm{MC}$ residues found in these areas are typically heterogeneous in distribution (Jenkins et al. 2001).

Manufacturing of explosives and their loading, assembling, packing into munitions for use in testing, training, and combat has impacted terrestrial and aquatic systems (Monteil-Rivera et al. 2009). Thousands of sites throughout the world are potentially contaminated, with MC in the soil, sediment, groundwater or the surface water of inland habitats, at concentrations that span several orders of magnitude (Talmage et al. 1999; Jenkins et al. 2001). Explosives loaded into munitions as well as fragments of explosives and munitions remaining following incomplete detonations may be present in surface soils and in aquatic habits.

\section{U.S. Military}

In the United States, unexploded ordnance (UXO) and discarded military munitions (DMM) are present at sites designated for Base Realignment and Closure (BRAC), at Formerly Used Defense Sites (FUDS), and at operational military ranges. Within the FUDS program, the U.S. Army Corps of Engineers (USACE) has identified more than 400 sites, totaling more than 10 million acres that potentially contain munitions in underwater environments. The U.S. Navy and U.S. Marine Corps' munitions response program (MRP) has identified an additional 33 sites containing munitions. The inventory includes sites that date back to the $18^{\text {th }}$ century and some that were used as recently as the 1990s (SERDP 2010).

\section{Underwater Munitions}

Military testing and training ranges may have both terrestrial and aquatic components. Although many aquatic environments encumbered with munitions have resulted from overshoots of land ranges, offshore areas 
also have been used as ranges. This is evident in many areas by simple inspection of the shoreline adjacent to target and practice ranges (McDonald 2008). Munitions present at these sites include inert and training rounds and also munitions that failed to function as designed and either remain unexploded (e.g., UXO) or that did not fully consume their explosive fill (e.g., low order rounds). Munitions and explosives of concern (MEC) may also be present in the underwater environment due to disposal, accidents and combat.

Underwater munitions sites encompass a variety of environmental conditions with complex characteristics and chemistry, such as depth, temperature, salinity, bathymetry, and sediment type, all of which can vary with time (SERDP 2010).

\section{Munitions in the Marine Environment}

The United States military has conducted open water disposal of a portion of both U.S. and foreign (captured) excess, obsolete, or unserviceable conventional and chemical munitions from at least as early as 1919 until 1970, at which time the DoD prohibited the practice. In 1972, Congress enacted the Marine Protection, Research, and Sanctuaries Act (Public Law 92-532) that generally prohibits sea disposal of all such material (Davis 2009).

Although the extent of biological receptors' exposure to $\mathrm{MC}$ in marine environments is largely unknown, the presence of munitions in aquatic environments is well-documented (Darrach et al. 1998; Dave 2003; Cusson et al. 2003; van Ham et al. 2007) and low concentrations of some explosive MC were measured in marine sediments (Darrach et al. 1998, Pascoe et al. 2010). It is anticipated that commonly used explosive fillers such as TNT, RDX and ammonium picrate may leak from underwater corroded, or breached, munitions as well as from fragments of explosives formulations that remain following low-order (incomplete) detonations (Wang et al. 2011).

\section{Other Stressors}

Other stressors, such as mechanical disturbance or unusual water quality parameters, can potentially contribute to adverse ecological effects. These other stressors need to be considered when evaluating the effects of $\mathrm{MC}$ on the aquatic environment. 


\section{Overview of Munitions Constituents}

Modern military explosives have an extensive history over the past 100 years, and terrestrial sites have been contaminated by (1) explosive manufacturing processes, (2) DMM, or (3) UXO. The U.S. Environmental Protection Agency (USEPA 1993) loosely classifies all "explosive waste" as energetic material, which can be further categorized as propellants, explosives, or pyrotechnics (PEP), based on typical use in military munitions.

Explosives are further classified as primary or secondary based on their susceptibility to initiation. Primary explosives, which include lead azide and lead styphnate, are highly susceptible to initiation. Primary explosives often are referred to as "initiating explosives" because they can be used to ignite secondary explosives (USEPA 1993).

Secondary explosives, which include TNT, RDX (cyclonite or Royal Demolition Explosive), and High Melting Explosive (HMX), are more prevalent at MEC sites than are primary explosives. Since they are formulated to detonate only under specific circumstances, secondary explosives often are used as main charge or boostering explosives. Secondary explosives are based on a binder and a crystalline explosive such as RDX. Secondary explosives also can be classified according to their chemical structure as nitroaromatics (e.g., TNT), and nitramines, which would include RDX (USEPA 1993).

This report deals almost exclusively with secondary explosives since they are present in munitions at much greater quantities than primary explosives. The report only addresses the aquatic ecotoxicology of explosives and propellants and related compounds. Most explosive chemicals used by the DoD are classified as nitroaromatics, nitramines or nitrate esters.

Among the nitroaromatics, TNT is widely used as an explosive, and DNT (2,4-dinitrotoluene) as a component of many single-base propellants. RDX and HMX are cyclic nitramines used in various explosives, and nitroglycerin and nitrocellulose are nitrate esters used in gun and some rocket propelants. Table 3.1 summarizes the energetic chemicals present in some military explosives. Many types of fillers used in munitions are composition 
explosives, consisting of two or more explosive compounds mixed to produce an explosive with more suitable characteristics for a particular application. Older energetic formulations contain compounds such as tetryl or ammonium picrate.

Table 3.1. Energetic chemicals present in military explosive and propellant formulations (USEPA 2012).

\begin{tabular}{|l|l|l|}
\hline $\begin{array}{l}\text { Compound or Explosive } \\
\text { Formulations }\end{array}$ & Uses & Chemical Ingredients \\
\hline Composition B & Artillery; mortar & $\begin{array}{l}\text { 60\% Military-grade RDX (Contains . 10\% HMX) } \\
\text { 39\% Military-grade TNT (Contains 1\% other TNT } \\
\text { isomers and DNTs); 1 \% wax }\end{array}$ \\
\hline Composition C4 & Demolition explosive & 91\% Military-grade RDX \\
\hline Tritonal & Air Force bombs & Military-grade TNT, aluminum \\
\hline Composition A4 & 40-mm grenades & Military-grade RDX \\
\hline TNT & Artillery & Military-grade TNT \\
\hline Composition H-6 & Navy and Marine bombs & Military-grade RDX and TNT, aluminum \\
\hline Octol & Antitank rockets & Military-grade HMX and TNT \\
\hline Explosive D & Naval projectiles & Ammonium Picrate \\
\hline
\end{tabular}

Information on the content of specific munitions may be found in Army and Navy manuals (e.g., US Army 1990, 1993; U.S. Navy 1987, 1999) and from online sources such as ORDATA (http://ordatamines.maic.jmu.edu/default.aspx). DoD employees can obtain detailed information about munitions through the Defense Ammunition Center's Munitions Items Disposition System (MIDAS) database. Access to MIDAS requires a Common Access Card.

With the exception of nitroglycerine, the major energetic compounds used by the DoD are solids at ambient temperatures. As a group, military-grade explosives have relatively low vapor pressure, low water solubility and hydrophobicity (Table 3.2).

The environmental fate of an MC is determined by its physical properties, such as solubility, density, melting point, vapor pressure, Henry's Law constant, and partitioning coefficients, as well as the physical properties of the aquatic environment. Physical parameters of the aquatic environment that influence the chemical fate of $\mathrm{MC}$ include temperature, $\mathrm{pH}$, salinity, dissolved oxygen, and the ionic strength of seawater. All of these factors in combination determine to what extent each compound will react in the environment. Possible chemical reactions in the aquatic environment include hydrolysis, photolysis, thermolysis, and oxidation. A review of the 
Table 3.2. Physicochemical properties of munitions constituents addressed in this report (USEPA 2012, unless specified).

\begin{tabular}{|c|c|c|c|c|}
\hline Common Name & $\begin{array}{l}\text { Chemical } \\
\text { Abstract Service } \\
\text { Number }\end{array}$ & $\begin{array}{l}\text { Molecular Weight } \\
\left(\mathrm{g} \mathrm{mol}^{-1}\right)\end{array}$ & $\begin{array}{l}\text { Water Solubility } \\
\text { at } 25^{\circ} \mathrm{C}\left(\mathrm{mg} \mathrm{L}^{-1}\right)\end{array}$ & $\begin{array}{l}\text { Octanol/Water } \\
\text { Partition Coeff. } \\
\text { (log Kow) }\end{array}$ \\
\hline TNT (2,4,6-trinitrotoluene) & $118-96-7$ & 227.13 & 130 & 1.6 \\
\hline $\begin{array}{l}\text { 1,3,5-TNB } \\
(1,3,5 \text {-trinitrobenzene) }\end{array}$ & $99-35-4$ & 213.1 & $385^{a}$ & $1.18^{a}$ \\
\hline $\begin{array}{l}\text { 2-Am-DNT } \\
\text { (2-amino-4,6-dinitrotoluene) }\end{array}$ & $35572-78-2$ & 197.17 & 42 & 1.94 \\
\hline $\begin{array}{l}\text { 4-Am-DNT } \\
\text { (4-amino-2,6-dinitrotoluene) }\end{array}$ & 19406-51-0 & 197.17 & 42 & 1.91 \\
\hline $\begin{array}{l}\text { 2,4-DANT } \\
\text { (2,4-diamino-6-nitrotoluene) }\end{array}$ & $6629-29-4$ & 167.17 & ND & $0.7^{b}$ \\
\hline $\begin{array}{l}\text { 2,6-DANT } \\
\text { (2,6-diamino-4-nitrotoluene) }\end{array}$ & $59229-75-3$ & 167.17 & ND & $0.67^{b}$ \\
\hline 2,4-DNT (2,4-dinitrotoluene) & $121-14-2$ & 182.15 & 270 & 1.98 \\
\hline 2,6-DNT (2,6-dinitrotoluene) & $606-20-2$ & 182.15 & 206 & 2.02 \\
\hline Tetryl (methyl-2,4,6-trinitrophenylnitramine) & $479-45-8$ & 287.17 & 75 & 2.04 \\
\hline Picric Acid & $88-89-1$ & 229.10 & 12800 & 1.33 \\
\hline Ammonium Picrate (Explosive D) & $131-74-8$ & 246.14 & $10,200^{c}$ & no data \\
\hline RDX (Royal Demolition Explosive) & $121-82-4$ & 222.26 & 56.3 & 0.90 \\
\hline HMX (High Melting Explosivef ${ }^{f}$ & 2691-41-0 & 296.16 & 4.5 & 0.17 \\
\hline Nitroguanidine (NQ) & $556-88-7$ & 104.07 & 4400 & -0.89 \\
\hline Nitroglycerin (NG) & $55-63-0$ & 227.11 & 1800 & 1.62 \\
\hline Nitrocellulose (NC) & 9004-70-0 & $10^{5}-10^{6}$ & insoluble & no data \\
\hline PETN (Pentaerythrite tetranitrate) & $78-11-5$ & 316.17 & $43^{d}$ & 3.71 \\
\hline DEGDN (Diethyleneglycoldinitrate) & $693-21-0$ & 196.12 & $4000^{a}$ & $0.98^{a}$ \\
\hline
\end{tabular}

ND - not determined, a from Rosenblatt et al. (1991), b from Elovitz and Weber (1999), c from Greenwald (1926), d This value is uncertain; range of cited values from 2.1 to $43 \mathrm{mg} \mathrm{L}-1$, e hexahydro-1,3,5-trinitro-1,3,5-triazine or cyclo-1,3,5-trimethylene-2,4,6trinitramine, also known as cyclonite, ${ }^{\mathrm{f}}$ Octahydro-1,3,5,7-tetranitro-1,3,5,7-tetrazocine

physicochemical properties for major explosives and a discussion of their environmental fate in the marine environment are provided in Noblis (2011). Singh et al. (2012) summarized information on the biodegradation and biotransformation pathways of major explosives. 
The following subsections provide basic background information on explosives and propellants for which data on toxicity and bioaccumulation in aquatic organisms are available.

\section{Nitroaromatics}

\section{2,4,6-trinitrotoluene (TNT)}

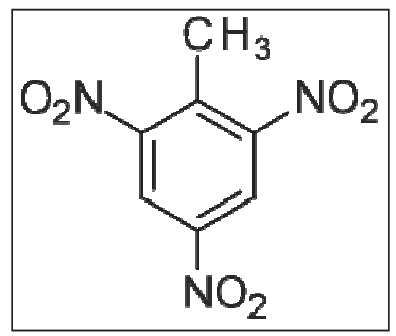

The U.S. military use of TNT began around 1912 typically in TNT only compositions, or mixed with powdered aluminum for enhanced blast effect. It is a stable explosive and is relatively insensitive to shock. Due to production limitations preceding both World War I and early World War II, TNT was blended with other energetics (e.g., Amatol is 50-80 \% ammonium nitrate and 50-20\% TNT) to stretch existing inventories and satisfy demands for large quantities of wartime explosives. TNT continues to be an explosive component of modern military munitions, although it is commonly mixed with other compounds (e.g., RDX). TNT is used in many explosive mixtures such as pentolite, tetrytol, torpex, tritonal, picratol, and Composition B.

At room temperature, TNT is a yellow solid. It is slightly soluble in water. Photochemical transformation (i.e., photolysis or photooxidation) of TNT in aqueous solutions has been investigated (O'Sullivan et al. 2011). When associated with organic matter in the water column, soil or sediment, TNT typically biotransforms, sometimes extensively, to reduced animated compounds, first to produce aminodinitrotoluene (Am-DNT) and preferentially 4-Am-DNT, with further reduction producing diaminonitrotoluene (DANT) and preferentially 2,4-DANT (Monteil-Rivera et al. 2009). Strong sorption to sediment organic matter not explained by hydrophobic partitioning has been reported for freshwater (Elovitz and Weber 1999) and marine sediment (Pennington et al. 2011). 


\section{1,3,5-trinitrobenzene $(1,3,5-T N B)$}

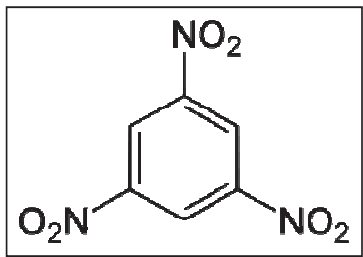

The compound 1,3,5-TNB has been primarily produced for use as an explosive. It can also be used to vulcanize natural rubber and as an acidbase indicator in the $\mathrm{pH}$ range of 12 . The compound $1,3,5$-TNB is also formed as a by-product of TNT manufacture and as a product of TNT environmental transformation, including its photolysis (Talmage et al. 1999).

At room temperature, 1,3,5-TNB is a clear to light yellow solid. It is slightly soluble in water. The compound 1,3,5-TNB appears to be resistant to photolysis; biotransformation of 1,3,5-TNB involves reduction of the nitro groups to form amino groups (Talmage et al. 1999). Extensive transformation of 1,3,5-TNB has been shown to occur in freshwater (Steevens et al. 2002; Lotufo and Farrar 2005) and marine sediment (Lotufo et al. 2001).

\section{2,4-and 2,6- Dinitrotoluene (2,4-DNT and 2,6-DNT)}

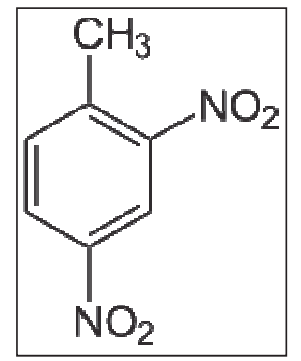

DNTs are a primary product used in propellants and are also formed as a byproduct during the TNT manufacturing process. Like TNT, DNTs can be reduced to the corresponding mono- or diamine derivatives (MonteilRivera et al. 2009). DNTs have also been associated, either as precursors or by-products, with the synthesis of polyurethane foams, coatings, and elastomers (USAPHC 2011). There are six isomers of DNT, the most common one is 2,4-DNT. Technical grade DNT is composed of 
approximately $75 \%$ 2,4-DNT, $20 \%$ 2,6-DNT, and $5 \%$ other isomers (USAPHC 2011).

The 2,4-DNT and 2,6-DNT isomers are yellow to reddish crystaline solids at room temperature and are moderately soluble in water. Reported low $\log \mathrm{K}_{\mathrm{oc}}$ (1.65 and 1.96 for 4-DNT and 2,6-DNT respectively) suggest low affinity for organic particulate matter (USEPA 2012). However, strong sorption of 2,4-DNT to soil organic matter has been reported (Singh et al. 2010).

Tetryl, Ammonium Picrate (Explosive D), and Picric Acid

\begin{tabular}{|c|c|c|}
\hline Tetryl & Ammonium Picrate & Picric Acid \\
\hline
\end{tabular}

Tetryl (methyl-N-2,4,6-trinitrophenylnitramine-) was first synthesized in 1877 and used since 1906 as an explosive in booster charges, detonator charges, and as a component of explosives mixtures (Tetrytols) during WWII (U.S. Army 1961). Since 1973, the use of tetryl by the US military has been gradually phased out in favor of plastic bonded RDX and HMX formulas (Talmage et al. 1999).

Picric acid (2,4,6-trinitrophenol), a strongly acidic phenol $\left(\mathrm{pK}_{\mathrm{a}}=0.38\right)$ was used as a military explosive starting in the 1880 . Due to a tendency to form shock-sensitive picrates in the presence of metals and for its corrosive effects on shell casings, picric acid as an explosive composition was largely discontinued during the first part of the $2 \mathrm{O}^{\text {th }}$ century in favor of other formulations (USEPA 1993).

Ammonium picrate (2,4,6-trinitrophenol ammonium salt or picric acid ammonium salt) is a salt formed by reacting picric acid and ammonia. First prepared in 1841, it was adopted for use as a bursting-charge explosive by the U.S. Navy in 1907. From 1917 to WWII, ammonium picrate, also known by the military formula designation "Explosive D," was a common explosive 
in virtually all calibers of armor-piercing ammunition due to its relative insensitivity to shock and friction (U.S. Army 1961). Picratol, a mixture consisting of $48 \%$ TNT and 52\% ammonium picrate, was developed during WWII to allow faster production of cast bomb and projectiles compared with traditional pressed shell methods required by Explosive D alone. Explosive D was gradually replaced by RDX blends such as Composition A toward the end of WWII and was of limited use in the post-war era.

Tetryl is a yellow crystalline solid powder material with low solubility in water. Picric acid is a colorless, yellow, or yellow-red crystalline powder. Ammonium picrate and picric acid are very soluble in water. When dissolved in water, ammonium picrate and picric acid dissociate to unstable picrate salts such as potassium picrate or picramic acid (2-amino-4,6dintrophenol).

Photolysis and hydrolysis are major environmental transformation processes for tetryl in aqueous media, as detailed in Talmage et al. (1999). In natural, sunlit waters, tetryl is transformed via photolysis and hydrolysis to several degradation products, including picric acid, which is the primary hydrolysis product of tetryl (Talmage et al. 1999). Information on the fate of tetryl in sediments is limited to a single paper (Nipper et al. 2002) that reported the formation of unknown breakdown products in sandy and finegrained marine sediments spiked with tetryl.

When dissolved in water, both ammonium picrate and picric acid dissociate to the picrate ion (Thorne and Jenkins 1997). Similar to TNT, picric acid is also degraded through reduction, and the microbial biodegradation of picric acid results in the formation of 2A-dinitrophenol (Monteil-Rivera 2009). Under aerobic conditions, the mono- or dinitrophenol can then be oxygenated with subsequent ring cleavage (Monteil-Rivera 2009). The fate of picric acid in marine sediments was investigated by Nipper et al. (2005). Transformation rates were highest in incubated, fine-grained sediment and major transformation products included 4-dinitrophenol, aminodinitrophenols (including picramic acid), 3,4-diaminophenol, amino nitrophenol, and nitrodiaminophenol. In the study by Dave et al. (2000), picric acid bound to sediment and resisted hydrolysis, biodegradation and photolysis. 


\section{Cyclic Nitramines}

RDX

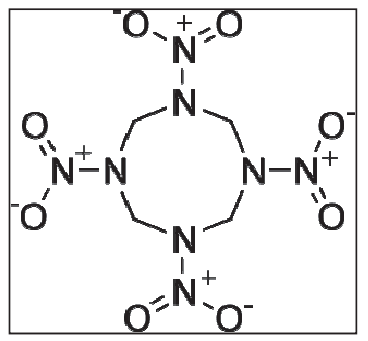

HMX

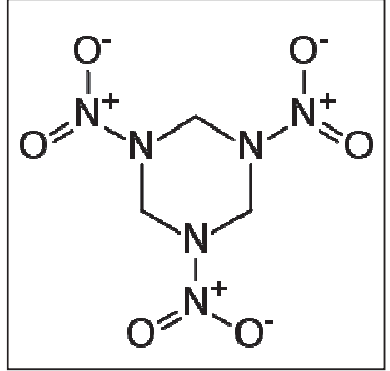

Commonly referred to as Royal Demolition Explosive, RDX (cyclo-1,3,5trimethylene-2,4,6-trinitramine, hexahydro-1,3,5-trinitro-1,3,5-triazine or cyclonite) was first synthesized for medicinal use in 1899. Although its value as an explosive was soon recognized, it was not until the early to mid-1940s that mass production techniques improved sufficiently enough to supply the necessary quantities for use in military formulations.

Commonly referred to as High Melting Explosive, HMX (octahydro1,3,5,7-tetranitro-1,3,5,7-tetrazocine) is a component of plastic explosives, solid fuel rocket propellants, and military munitions, as well as being an impurity of RDX.

At room temperature, both RDX and HMX are crystalline solids. Militarygrade RDX generally contains up to $10 \% \mathrm{HMX}$ as an impurity of the manufacturing process (Talmage et al. 1999). RDX has low solubility in water, and HMX is even less soluble. Their low $\mathrm{K}_{\mathrm{ow}}$ indicates their hydrophobicity.

The biodegradation of RDX occurs under both aerobic and anaerobic conditions but the anaerobic process is significantly faster (Monteil-Rivera et al. 2009). Unlike nitroaromatic explosives, RDX undergoes ring cleavage and mineralization under both aerobic and anaerobic conditions (Monteil-Rivera et al. 2009). Aerobically, it has been found that microbial cleavage of one of the $\mathrm{N}-\mathrm{NO}_{2}$ bonds produces unstable intermediates (Monteil-Rivera et al. 2009). Subsequent degradation reactions are largely governed by the interactions of the newly formed intermediates with water and eventually produce $\mathrm{NO}_{2}^{-}, \mathrm{N}_{2} \mathrm{O}, \mathrm{NH}_{3}, \mathrm{HCHO}$, and $\mathrm{HCOOH}$ (MonteilRivera et al. 2009). Anaerobically, sequential reduction of the nitro groups 
to produce mono-, di- and trinitroso derivatives (MNX, DNX and TNX, respectively) is most frequently observed. The nitroso derivatives may be further transformed to produce the unstable hydroxylamine derivatives, leading to ring cleavage (Crocker et al. 2006). Degradation of HMX does not appear to occur in the field under aerobic conditions, with half-lives in the environment spanning decades (Clausen and Korte 2011). However, anaerobic degradation of HMX has been observed and described (MonteilRivera et al. 2009).

RDX and HMX have lower water solubility and significantly less binding affinity with soil organic matter than TNT; RDX and HMX are only poorly immobilized by soils (Monteil-Rivera et al. 2009). Extensive transformation of RDX occurred following spiking to fine-grained sediment (Lotufo et al. 2001; Pennington et al. 2011), but the transformation products were not identified. Different from the fate of RDX in organically-rich sediment, transformation was minimal in sandy sediment (Rosen and Lotufo 2005).

\section{Other Energetic Compounds}

\section{Nitroguanidine}

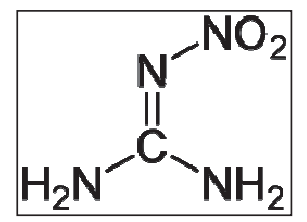

Nitroguanidine is also known by its military designation - NQ. It is a nitroimino compound that exists in two tautomeric forms; the tautomer form depicted above is predominant. It is a stable explosive compound, and when combined with nitrocellulose and nitroglycerin, forms the socalled triple-based propellant mixture.

Nitroguanidine is water soluble and may enter the environment via discharge streams from handling facilities (Kaplan et al. 1982). In addition, it has been found at army ranges as a result of live-fire training (Clausen 2011). Degradation of nitroguanidine was shown to be negligible in activated sludge under aerobic conditions and in sterile sludge under reducing conditions (Kaplan et al. 1982). 


\section{Nitrate Esters: nitroglycerin, nitrocellulose, PETN and DEGDN (diethylene glycol dinitrate)}

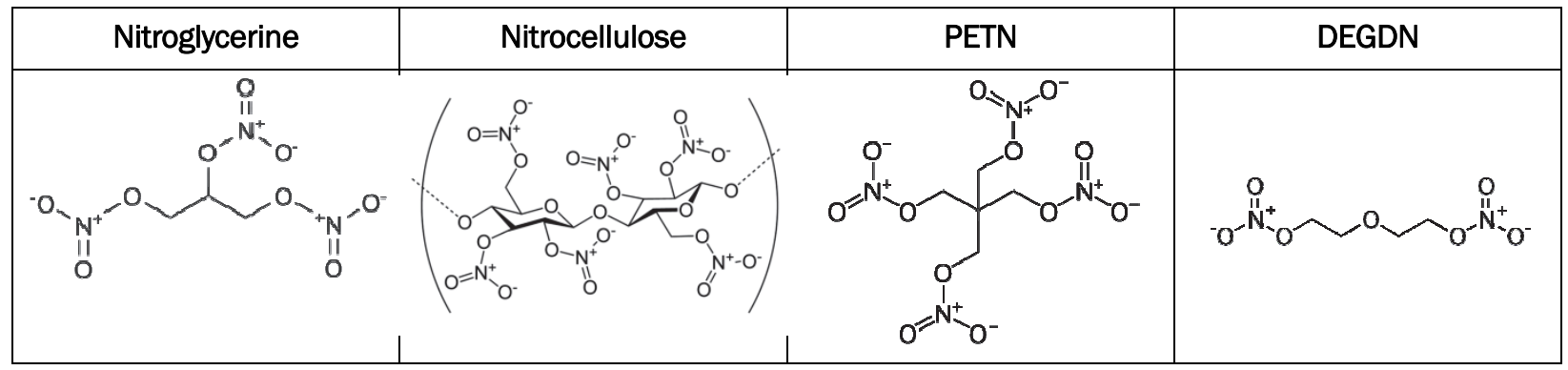

Nitrocellulose is a highly flammable compound formed by nitrating cellulose through exposure to nitric acid or another powerful nitrating agent. When used as a propellant or low-order explosive, it is also known as guncotton. Nitrocellulose occurs as a particulate that is essentially insoluble in water (Bentley et al. 1977d) and very recalcitrant to microbial degradation (Podlipná et al. 2008).

Nitroglycerin or glycerol trinitrate (propane-1,2,3-triyl trinitrate) is used in manufacture of dynamite, gunpowder, and rocket propellants, and as a therapeutic agent (Rocheleau et al. 2011). Double-base propellants used within newer small-arms ammunition typically contain up to $84 \%$ nitrocellulose, with $10 \%$ nitroglycerin, a stabilizer, and up to $6 \%$ filler compounds. The combination of nitroglycerin, nitrocellulose and nitroguanidine forms a triple-base propellant mixture. Nitroglycerin can be released into the environment at firing positions, and in the target areas due to low-order detonation of propellant (Rocheleau et al. 2011). Nitroglycerin is water soluble, and may enter and persist in freshwater environments (USACHPPM 2001). Microbial degradation of nitroglycerin has been reported (Podlipná et al. 2008).

PETN, or pentyl (1,3-dinitrato-2,2-bis(nitratomethyl)propane , or pentaerythritol tetranitrate) is used in some booster charges, demolition explosives, and initiating explosives such as primer cord. PETN is considered practically insoluble in water, not prone to hydrolyze at ambient temperature and recalcitrant to microbial degradation (Podlipná et al. 2008).

DEGDN is an explosive ingredient (60-80\%) in dynamite along with nitroglycerine (40-20\%) and has been used in propellant mixtures as a plasticizer (Fisher et al. 1989). DEGDN is a colorless, odorless, viscous, oily liquid. Environmental fate studies indicate that DEGDN is a stable compound once dissolved in water (Spanggord et al. 1985). 


\section{Aqueous Toxicity of Munitions Constituents to Fish and Aquatic Invertebrates}

This section summarizes the available data on the toxicity of $\mathrm{MC}$ to fish and aquatic invertebrates. The data were generated through laboratory exposures of test organisms to water spiked with energetic MC.

\section{Overview}

To support the assessment of risk associated with the presence of explosives in aquatic environments, contaminant-spiked water is often used in laboratory experiments to derive toxicity data for a variety of freshwater and marine species and endpoints. In order to bracket the toxic range and derive toxicity benchmarks, most studies involved the use of exposure concentrations significantly higher than those expected in the environment. Broad overviews of the effects of explosive compounds to aquatic organisms are provided by Talmage et al. (1999), Juhasz and Naidu (2007) and more recently by Nipper et al. (2009) who provided a compilation of available aquatic toxicity data expanded to include nitroaromatic and nitrophenolic compounds that are not considered MC or their transformation products. Although most investigations of the aqueous toxicity of explosives and related compounds were performed using freshwater organisms, toxicity data for a variety of energetic compounds were generated using marine species.

Several studies reported the toxicity of MC to a variety of freshwater fish, including fathead minnows, channel catfish, and zebrafish (reviewed in Talmage et al. 1999 and Nipper et al. 2009; see also Mukhi et al. 2005; Mukhi and Patino 2008; Gust et al. 2011), as well as to frog larvae (Saka et al. 2004; Paden et al. 2011). In contrast, adverse biological effects of MC were reported for only two marine fish species (Nipper et al. 2001; Lotufo et al. 2010a,b,c). In addition, cellular-level effects of TNT on a catadromous eel were also reported (Della Torre et al. 2008a,b,c).

The aqueous toxicity of MC to freshwater invertebrates has been reported for a variety of freshwater species, including amphipods, midges, rotifers, cladocerans and oligochaetes (reviewed in Talmage et al. 1999 and Nipper 
et al. 2009). Compared to studies with freshwater organisms, fewer studies addressed the effects of MC to marine invertebrates. Organisms used as test species included larval sea urchins, mysid shrimp (Nipper et al. 2001), larval and adult bivalves (Won et al. 1976; Goodfellow et al., 1983; Rosen and Lotufo 2007a), copepods (Won et al. 1976; Ek et al. 2006, 2007, 2008; Dave 2003; Dave et al. 2000), meiobenthic polychaete (Nipper et al. 2001) and macroalga spores (Nipper et al. 2001). The National Oceanic and Atmospheric Administration is currently investigating toxicity of several explosive compounds to corals.

Most of the studies cited above reported lethal effects. Reproductive effects of MC were reported for several species of freshwater fish (Bailey et al. 1985; Burton et al. 1994; Mukhi and Patino 2008) and invertebrates (Bailey et al. 1985; Peters et al. 1991; Snell and Moffat 1992; Conder et al. 2004a). Reports of sublethal effects for marine invertebrates included impacts to larval development of bivalves and sea urchins (Nipper et al. 2001; Rosen and Lotufo. 2007a) and polychaete reproduction (Nipper et al. 2001). Comparisons of lethal and sublethal endpoints for various MC suggest that the use of sublethal endpoints could be useful for the interpretation of potential long-term environmental effects of those chemicals, as concentrations associated with reproductive effects were lower, sometimes substantially, than those promoting mortality (Nipper et al. 2009).

\section{Fate of MC in exposure water during toxicity testing}

When fish and adult mussels were exposed to TNT using a static-renewal method in which the TNT-spiked water was exchanged daily, a large fraction of the TNT transformed to Am-DNTs (Lotufo et al. 2010a; Rosen and Lotufo 2007a). In addition, loss of nitroaromatic compounds also occurred in those studies, as the sum concentration of TNT, Am-DNTs and DANTs (referred to as SumTNT) decreased over the 24-h period between TNT-spiked water exchanges. As an example, the temporal pattern of change in SumTNT concentrations for different treatments of a 10-d sheepshead minnow static-renewal and exposure is shown in Figure 4.1. The percent of loss of the SumTNT tended to increase with decreasing concentrations. A similar pattern of temporal changes was observed in the adult mussel experiment. Loss of compounds was likely enhanced by the presence of the organisms themselves and the accumulation of food in the beaker (fish exposure only) and likely occurred as a result of volatilization, binding to food, as well as transformation to unidentified products. Transformation of TNT and decline of SumTNT concentrations between 
water exchanges in static-renewal toxicity tests were also observed in other studies (Carr and Nipper 2000; Nipper et al. 2001; Conder et al. 2004b; Ownby et al. 2005; Yoo et al. 2006). In contrast, virtually no transformation or losses of TNT occurred when exposures were conducted with no feeding, such as mussel embryo exposure to TNT (Rosen et al. 2007a).

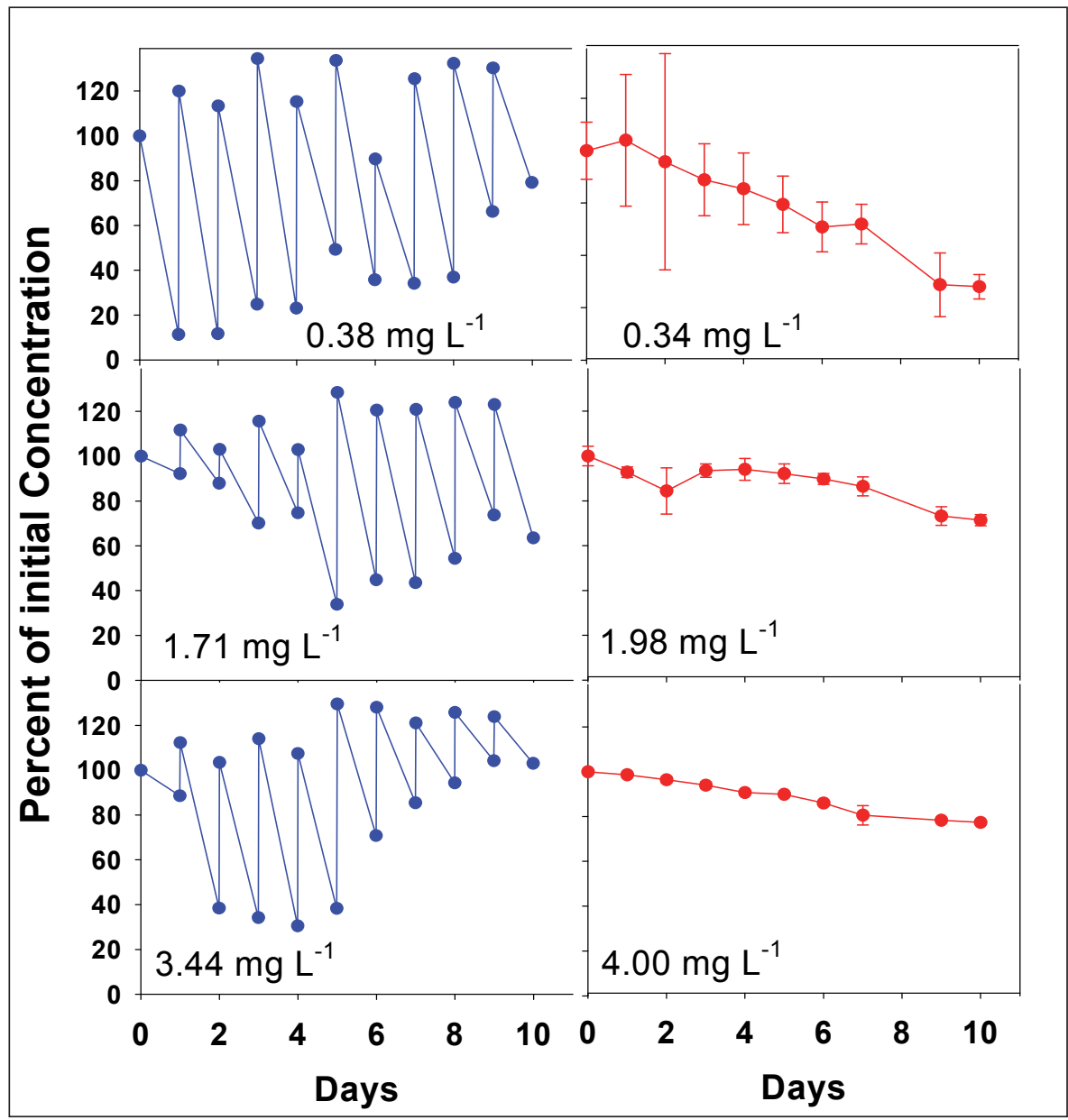

Figure 4.1. Temporal change in aqueous concentrations of SumTNT expressed as a percent of the initial concentration during the TNT 10-d static renewal experiment (left) and the TNT 10-d constant exposure experiment (right) (from Lotufo et al. 2010a).

In contrast with the static-renewal experiment, TNT exposures conducted using a diluter system that delivered spiked water every hour maintained stable concentrations over $24 \mathrm{~h}$ time intervals in experiments with sheepshead minnows (Lotufo et al. 2010a). However, the exposure water concentration declined gradually during the course of the 10-d experiment (Figure 4.1). Despite frequent turnover, enhancement of the microbial population due to the build-up of food and fish fecal detritus in the tanks likely promoted transformation of the TNT delivered to the exposure 
chambers. The use of a diluter system is recommended and feeding should be avoided whenever possible in aqueous toxicity evaluations of TNT.

During toxicity tests using fathead minnows, transformation of $1,3,5$-TNB dinitroaniles (or amino-dinitrobenzenes) occurred, leading to the sometimes dramatic decrease in the parent compound concentration during the 24-h period between water exchange events (Lotufo et al. 2010a). However, the concentration decrease for 2-Am-DNT and 2,4-DANT between water exchange events was minimal in similar experiments (Lotufo et al. 2010a). Therefore, any transformation or loss of those compounds was negligible. Persistence of Am-DNTs and DANTs in experimental systems has also been reported for sediment (Lotufo and Farrar 2005), soils (Lachance et al. 2004) and water (Conder et al. 2004b). The extensive transformation of $1,3,5$-TNB was surprising since $1,3,5$-TNB is considered environmentally persistent, not readily biodegradable, and with a high propensity to contaminate groundwater near production waste disposal sites (Reddy et al. 1997). While static-renewal exposures appear appropriate for toxicity evaluation of Am-DNTs and DANTs, the use of a diluter system is recommended for toxicity evaluation of $1,3,5$-TNB and the feeding of test organisms should be avoided whenever possible.

During aqueous exposures of sheepshead minnows to RDX (Lotufo et al. 2010b), the concentration of HMX in the exposure solutions represented approximately $8 \%$ of the sum concentration of RDX and HMX, corresponding to the reported concentration of HMX in the military grade RDX used in this study. No RDX or HMX transformation products were detected in the exposure solutions. Only negligible $(<5 \%)$ changes in RDX and HMX concentrations occurred between water exchanges in all experiments. Static-renewal exposures seem appropriate for the toxicity evaluation of RDX and HMX and feeding does not seem to promote the breakdown of parent compound as observed for TNT and 1,3,5-TNB.

Nipper et al. (2001) investigated the toxicity of eight MC to five marine species from different phyla. Chemical analysis for the $\mathrm{MC}$ at the beginning and end of each test indicated that some compounds were very stable, whereas others were lost in large amounts during the test exposure periods. The percent of loss of the most degradable chemicals tended to increase with decreasing concentrations. Overall, the compounds with three and four nitro groups (TNT, 1,3,5-TNB, and tetryl) exhibited higher loss than those with two nitro groups (1,3-DNB and 2,4- and 2,6-DNT). Picric acid and 
RDX exhibited little or no loss. The use of a diluter system is recommended and feeding should be avoided whenever possible in aqueous toxicity evaluations of TNT, 1,3,5-TNB, and tetryl.

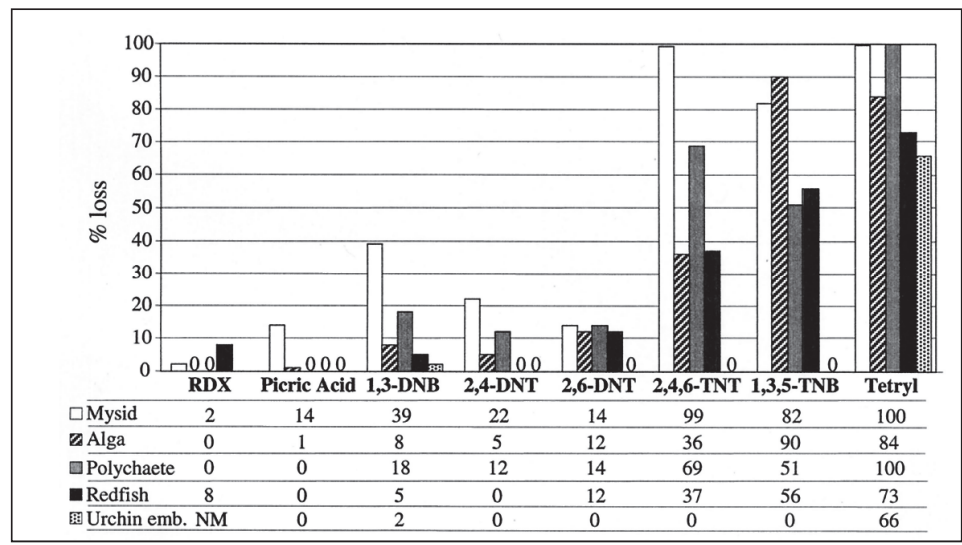

Figure 4.2. Mean percent MC lost in all test concentrations during aqueous exposures (from Nipper et al. 2001). NM - not measured at test termination.

\section{Toxicity of nitroaromatic MC}

Numerous aquatic toxicity studies have reported that TNT causes decreased survival in a number of aquatic invertebrate and fish species, typically at concentrations ranging from 1 to $10 \mathrm{mg} \mathrm{L}^{-1}$ (Nipper et al. 2009). The lethal toxicity of TNT to marine fish and invertebrates is summarized and compared using median lethal concentration $\left(\mathrm{LC}_{50}\right)$ values (Table 4.1).

Exposure of the marine species Sciaenops ocellatus (redfish) to TNT for 2 days during the hatching period (Nipper et al. 2001) generated an $\mathrm{LC}_{50}$ value higher than that reported for Cyprinodon variegatus (sheepshead minnow) exposed for 5 days. Toxicity data for longer exposure duration is not available for redfish.

Most invertebrates investigated exhibited similar sensitivities to TNT and overall less sensitivity than fish (Table 4.1; Figure 4.3). Mysid shrimp were the most sensitive species, while adult mussels were the most tolerant among the invertebrate species investigated. The relative high tolerance of adult mussels has also been observed for other contaminant classes. For example, $96 \mathrm{~h} \mathrm{LC}_{50}$ values for several organophosphorus pesticides ranged from 23 to $>56 \mathrm{mg} \mathrm{L}^{-1}$ for $M$. galloprovincialis, while $\mathrm{LC}_{50}$ S were generally 3-4 orders of magnitude lower in comparable exposures with fish (Serrano et al. 1995). 
Table 4.1. Lethal toxicity data for marine fish and invertebrates exposed to nitroaromatic MC.

\begin{tabular}{|c|c|c|c|c|}
\hline Species & MC & Duration $(d)$ & $\mathrm{LC}_{50}\left(\mathrm{mg} \mathrm{L}^{-1}\right)$ & Reference \\
\hline \multirow{5}{*}{$\begin{array}{l}\text { Sheepshead minnow } \\
\text { Cyprinodon variegatus }\end{array}$} & TNT & 10 & 2.3 & Lotufo et al. $2010 a$ \\
\hline & TNT & 5 & 1.7 & Lotufo et al. $2010 a$ \\
\hline & $\begin{array}{l}\text { 2-Am- } \\
\text { DNT }\end{array}$ & 5 & 8.6 & Lotufo et al. $2010 a$ \\
\hline & 2,4-DANT & 5 & \begin{tabular}{|l|} 
No lethal effects at \\
$50.3 \mathrm{mg} \mathrm{L}^{-1} \mathrm{a}$ \\
\end{tabular} & Lotufo et al. $2010 a$ \\
\hline & \begin{tabular}{|l}
$1,3,5-$ \\
TNB
\end{tabular} & 5 & 1.2 & Lotufo et al. $2010 a$ \\
\hline \multirow{4}{*}{$\begin{array}{l}\text { Redfish } \\
\text { Sciaenops ocellatus }\end{array}$} & TNT & 2 & 7.6 & Nipper et al. 2001 \\
\hline & 2,4-DNT & 2 & 48 & Nipper et al. 2001 \\
\hline & 2,6-DNT & 2 & 28 & Nipper et al. 2001 \\
\hline & $\begin{array}{l}\text { 1,3,5- } \\
\text { TNB }\end{array}$ & 2 & 1.2 & Nipper et al. 2001 \\
\hline $\begin{array}{l}\text { Oyster (larvae) } \\
\text { Crassostrea gigas }\end{array}$ & TNT & 4 & 8.2 & Won et al. 1976 \\
\hline $\begin{array}{l}\text { Mussel (adult) } \\
\text { M. galloprovincialis }\end{array}$ & TNT & 4 & 19.5 & Rosen and Lotufo $2007 a$ \\
\hline \multirow{4}{*}{$\begin{array}{l}\text { Mysid shrimp } \\
\text { Americamysis bahia }\end{array}$} & TNT & 4 & 0.98 & Nipper et al. 2001 \\
\hline & 2,4-DNT & 4 & 4.4 & Nipper et al. 2001 \\
\hline & 2,6-DNT & 4 & 5.0 & Nipper et al. 2001 \\
\hline & $\begin{array}{l}\text { 1,3,5- } \\
\text { TNB }\end{array}$ & 4 & 0.74 & Nipper et al. 2001 \\
\hline \multirow{4}{*}{$\begin{array}{l}\text { Copepod } \\
\text { Tigriopus californicus }\end{array}$} & TNT & 3 & 5.3 & Won et al. 1976 \\
\hline & $\begin{array}{l}\text { 2-Am- } \\
\text { DNT }\end{array}$ & 3 & $\begin{array}{l}\text { No lethal effects at } \\
50 \mathrm{mg} \mathrm{L}-1 \mathrm{a}\end{array}$ & Won et al. 1976 \\
\hline & 2,4-DANT & 3 & $\begin{array}{l}\text { No lethal effects at } \\
50 \mathrm{mg} \mathrm{L}^{-1} \mathrm{a}\end{array}$ & Won et al. 1976 \\
\hline & 2,6-DANT & 3 & $\begin{array}{l}\text { No lethal effects at } \\
50 \mathrm{mg} \mathrm{L} \mathrm{L}^{-1 \mathrm{a}}\end{array}$ & Won et al. 1976 \\
\hline \multirow[t]{2}{*}{$\begin{array}{l}\text { Copepod } \\
\text { Nitocra spinipes }\end{array}$} & TNT & 4 & 7.6 & Dave et al. 2000 \\
\hline & 2,4-DNT & 4 & 17 & Dave et al. 2000 \\
\hline \multirow{4}{*}{$\begin{array}{l}\text { Polychaete } \\
\text { Dinophilus gyrociliatus }\end{array}$} & TNT & 7 & 5.6 & Nipper et al. 2001 \\
\hline & 2,4-DNT & 7 & 20 & Nipper et al. 2001 \\
\hline & 2,6-DNT & 7 & 13 & Nipper et al. 2001 \\
\hline & \begin{tabular}{|l}
$1,3,5-$ \\
TNB \\
\end{tabular} & 7 & 1.6 & Nipper et al. 2001 \\
\hline $\begin{array}{l}\text { Amphipod } \\
\text { Eohaustorius estuarius }\end{array}$ & TNT & 4 & 4.5 & Rosen and Lotufo, unpublished \\
\hline $\begin{array}{l}\text { Amphipod } \\
\text { Leptocheirus plumulosus }\end{array}$ & TNT & 4 & 3.6 & Rosen and Lotufo, unpublished \\
\hline
\end{tabular}

$\mathrm{LC}_{50}$ - Median lethal concentration, a Highest concentration tested. 


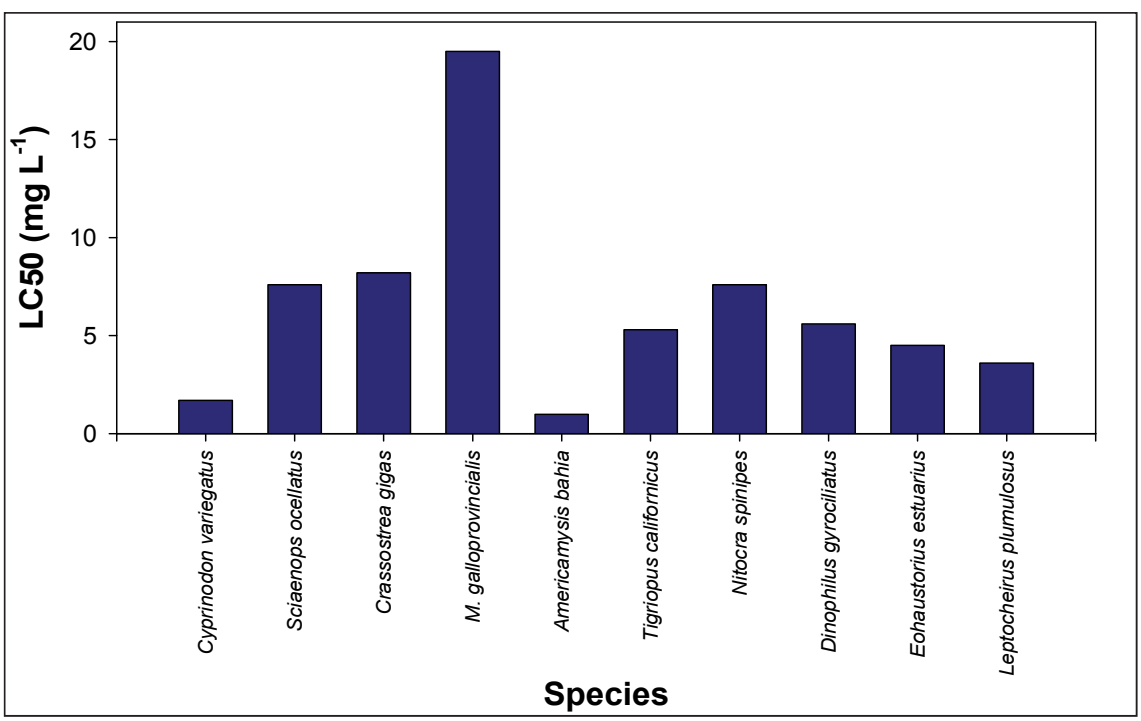

Figure 4.3. Lethal toxicity data for marine fish and invertebrates exposed to TNT. LC 50 - median lethal concentration. See Table 4.1 for references.

The TNT acute toxicity values derived for marine fish and invertebrates are comparable to those reported for freshwater organisms (Table 4.2), suggesting relatively similar sensitivity among all aquatic fish and invertebrates.

The toxicity of TNT was compared to that of its major transformation products 1,3,5-TNB, 2-Am-DNT and 2,4-DANT using sheepshead minnows (Figure 4.4) (Lotufo et al. 2010a). Nitro-reduction appears to decrease the toxicity of TNT to sheepshead minnows, as the $\mathrm{LC}_{50}$ for the mono-aminated compound 2-Am-DNT was approximately 4 times higher than that for TNT. Further amination appears to decrease toxicity even more dramatically, as the highest tested concentration for 2,4-DANT was 20 times higher than the $\mathrm{LC}_{50}$ for TNT and yet that concentration failed to promote significant mortality (Table 4.1).

Freshwater species varied in their relative sensitivity to lethal toxicity of TNT and its major aminated transformation products (Table 4.3). The toxicity of 2-Am-DNT was lower than that of TNT for fathead minnows. For larvae of the frog Xenopus laevis, the toxicity of 2-Am-DNT and 4Am-DNT were also lower than that of TNT. For invertebrates, 2-Am-DNT exhibited toxicity similar to that of TNT. The compound 2,4-DANT was more toxic than TNT for Hyalella azteca but substantially less toxic to Chironomus dilutus (formerly known as $C$. tentans). The compound 1,3,5TNB was more toxic than TNT to sheepshead minnows and fathead minnows, as well as for the invertebrates Daphnia magna and Hyalella azteca, but was almost identically lethal as TNT to Chironomus dilutus. 
Table 4.2. Lethal toxicity data for freshwater fish, amphibians and invertebrates exposed to TNT.

\begin{tabular}{|c|c|c|c|}
\hline Species & Duration (d) & $\mathrm{LC}_{50}\left(\mathrm{mg} \mathrm{L}^{-1}\right)$ & Reference \\
\hline $\begin{array}{l}\text { Bullfrog (tadpole) } \\
\text { Rana catesbeiana }\end{array}$ & 4 & 40.3 & Paden et al. 2011 \\
\hline $\begin{array}{l}\text { Frog (tadpole) } \\
\text { Xenopus laevis }\end{array}$ & 4 & 3.8 & Saka et al. 2004 \\
\hline $\begin{array}{l}\text { Fathead minnow } \\
\text { Pimephales promelas }\end{array}$ & 10 & 2.2 & Yoo et al. 2006 \\
\hline Pimephales promelas & 4 & 2.9 & Liu et al. $1983 a$ \\
\hline $\begin{array}{l}\text { Bluegill } \\
\text { Lepomis macrochirus }\end{array}$ & 4 & 2.6 & Liu et al. $1983 a$ \\
\hline $\begin{array}{l}\text { Rainbow trout } \\
\text { Oncorhynchus mykiss }\end{array}$ & 4 & 0.8 & Liu et al. $1983 b$ \\
\hline $\begin{array}{l}\text { Channel catfish } \\
\text { Ictalurus punctatus }\end{array}$ & 4 & 2.4 & Liu et al. $1983 a$ \\
\hline $\begin{array}{l}\text { Amphipod } \\
\text { Hyalella azteca }\end{array}$ & 4 & 3.6 & $\begin{array}{l}\text { Sims and Steevens } \\
2008\end{array}$ \\
\hline $\begin{array}{l}\text { Oligochaete } \\
\text { Lumbriculus variegatus }\end{array}$ & 2 & 5.2 & Liu et al. $1983 b$ \\
\hline $\begin{array}{l}\text { Oligochaete } \\
\text { Tubifex tubifex }\end{array}$ & 4 & 7.7 a & Conder et al. 2004c \\
\hline $\begin{array}{l}\text { Cladoceran } \\
\text { Daphnia magna }\end{array}$ & 4 & 5.1 & Johnson et al. 1994 \\
\hline $\begin{array}{l}\text { Cladoceran } \\
\text { Ceriodaphnia dubia }\end{array}$ & 7 & $\begin{array}{l}\text { No lethal effects at } \\
6.1 \mathrm{mg} \mathrm{L}^{-1 \mathrm{~b}}\end{array}$ & Griest et al. 1998 \\
\hline $\begin{array}{l}\text { Midge (larva) } \\
\text { Chironomus tentans }\end{array}$ & 4 & 1.9 & Conder et al. 2004c \\
\hline $\begin{array}{l}\text { Rotifer } \\
\text { Branchionus calyciflorus }\end{array}$ & 1 & 5.6 & Toussaint et al. 1995 \\
\hline
\end{tabular}

LC50 - median lethal concentration, a Based on nominal concentrations, b Highest concentration tested. 


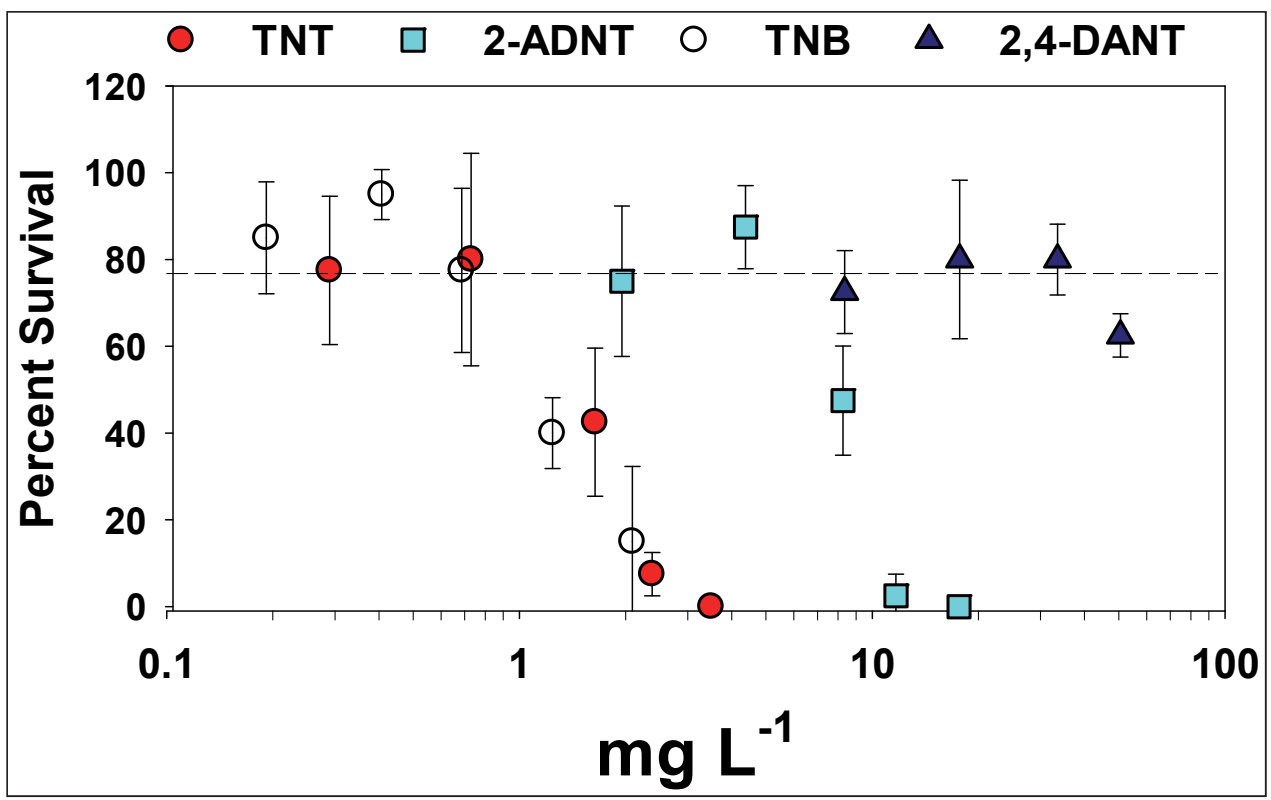

Figure 4.4. Mean percent survival of juvenile sheepshead minnows (Cyprinodon variegatus) at termination of the 5-d exposure to aqueous exposures to TNT, 2-AmDNT, 1,3,5-TNB and 2,4-DANT (on log scale) (from Lotufo et al. 2010a). Error bars are \pm 1 standard deviation. The dashed line indicates mean control percent survival.

Table 4.3. Lethal toxicity data for fish, a tadpole and aquatic invertebrates exposed to TNT, Am-DNTs, DANTs, and 1,3,5-TNB.

\begin{tabular}{|c|c|c|c|c|c|c|c|c|}
\hline \multirow[b]{2}{*}{ Species } & \multirow[b]{2}{*}{$\begin{array}{l}\text { Duration } \\
\text { (d) }\end{array}$} & \multicolumn{6}{|c|}{$\mathrm{LC}_{50}\left(\mathrm{mg} \mathrm{L}^{-1}\right)$} & \multirow[b]{2}{*}{ Reference } \\
\hline & & TNT & $\begin{array}{l}\text { 2-Am- } \\
\text { DNT }\end{array}$ & $\begin{array}{l}\text { 4-Am- } \\
\text { DNT }\end{array}$ & 2,4-DANT & $\begin{array}{l}2,6- \\
\text { DANT }\end{array}$ & $\begin{array}{l}\text { 1,3,5- } \\
\text { TNB }\end{array}$ & \\
\hline \multicolumn{9}{|c|}{ Marine species } \\
\hline $\begin{array}{l}\text { Sheepshead } \\
\text { minnow } \\
\text { Cyprinodon } \\
\text { variegatus }\end{array}$ & 5 & 1.7 & 8.6 & ND & $\begin{array}{l}\text { No effect } \\
\text { at } 50.3 \\
\mathrm{mg} \mathrm{L}^{-1} \text { a }\end{array}$ & ND & 1.2 & $\begin{array}{l}\text { Lotufo et al. } \\
2010 a\end{array}$ \\
\hline \multicolumn{9}{|c|}{ Freshwater species } \\
\hline $\begin{array}{l}\text { Frog tadpole } \\
\text { Xenopus laevis }\end{array}$ & 4 & 3.8 & 32.7 & 22.7 & ND & ND & ND & $\begin{array}{l}\text { Saka et al. } \\
2004\end{array}$ \\
\hline $\begin{array}{l}\text { Fathead } \\
\text { minnow } \\
\text { Pimephales } \\
\text { promelas }\end{array}$ & 4 & 2.4 & 14.8 & 6.9 & ND & ND & 1.0 & $\begin{array}{l}\text { Pearson et al. } \\
1979\end{array}$ \\
\hline \multirow{2}{*}{$\begin{array}{l}\text { Cladoceran } \\
\text { Daphnia } \\
\text { magna }\end{array}$} & 2 & 11.9 & 4.5 & 5.2 & ND & ND & 2.7 & $\begin{array}{l}\text { Pearson et. al. } \\
1979\end{array}$ \\
\hline & 4 & 5.1 & 1.1 & 5.1 & ND & ND & ND & $\begin{array}{l}\text { Johnson et al. } \\
1994\end{array}$ \\
\hline $\begin{array}{l}\text { Cladoceran } \\
\text { Ceriodaphnia } \\
\text { dubia }\end{array}$ & 7 & $\begin{array}{l}\text { No } \\
\text { effect at } \\
6.0 \mathrm{mg} \\
\mathrm{L}^{-1} \mathrm{a}\end{array}$ & 4.9 & 6.6 & $\begin{array}{l}\text { No effect } \\
\text { at } 0.2 \\
\text { mg L-1 a }\end{array}$ & $\begin{array}{l}\text { No } \\
\text { effect at } \\
2.0 \mathrm{mg} \\
\mathrm{L}^{-1} \mathrm{a}\end{array}$ & $\begin{array}{l}\text { No } \\
\text { effect at } \\
2.0 \mathrm{mg} \\
\mathrm{L}^{-1} \mathrm{a}\end{array}$ & $\begin{array}{l}\text { Griest et al. } \\
1998\end{array}$ \\
\hline
\end{tabular}




\begin{tabular}{|c|c|c|c|c|c|c|c|c|}
\hline \multirow[b]{2}{*}{ Species } & \multirow[b]{2}{*}{$\begin{array}{l}\text { Duration } \\
\text { (d) }\end{array}$} & \multicolumn{6}{|c|}{$\mathrm{LC}_{50}\left(\mathrm{mg} \mathrm{L}^{-1}\right)$} & \multirow[b]{2}{*}{ Reference } \\
\hline & & TNT & $\begin{array}{l}\text { 2-Am- } \\
\text { DNT }\end{array}$ & $\begin{array}{l}\text { 4-Am- } \\
\text { DNT }\end{array}$ & 2,4-DANT & $\begin{array}{l}2,6- \\
\text { DANT }\end{array}$ & $\begin{array}{l}\text { 1,3,5- } \\
\text { TNB }\end{array}$ & \\
\hline $\begin{array}{l}\text { Amphipod } \\
\text { Hyalella } \\
\text { azteca }\end{array}$ & 4 & 3.6 & 3.8 & 9.2 & 1.7 & ND & 2.3 & $\begin{array}{l}\text { Sims and } \\
\text { Steevens } \\
2008\end{array}$ \\
\hline $\begin{array}{l}\text { Midge (larva) } \\
\text { Chironomus } \\
\text { dilutus }\end{array}$ & 4 & 1.9 & 3.3 & ND & 33.3 & ND & 2.2 & $\begin{array}{l}\text { Lotufo, } \\
\text { unpublished }\end{array}$ \\
\hline
\end{tabular}

LC50 - median lethal concentration, ND - not determined, a Highest concentration tested, b The No observed effect concentration was 3.0 and lowest observed effect concentration was $6.0 \mathrm{mg} \mathrm{L}^{-1}$.

The sublethal effects of TNT, examined as mussel larval development, occurred at a concentration substantially lower than the lethal median concentration determined for adult mussels (Rosen and Lotufo 2007a) (Table 4.4). Mussel larvae were also more sensitive to the developmental effects of TNT than sea urchin embryos (Davenport et al. 1994, Nipper et al. 2001, Rosen and Lotufo 2007a) (Table 4.4). Embryo-larval development involves critical phases of differentiation (e.g., gastrulation), which are often more sensitive than other life stages. The development of functional mussel byssal threads has been previously investigated as a sublethal endpoint (Serrano et al. 1995). After $48 \mathrm{~h}$ of TNT exposure, the byssal thread development endpoint was more sensitive than survival by a factor of 3 , indicating that functional byssi is a relatively sensitive sublethal endpoint for TNT (Rosen and Lotufo 2007a). Sublethal effects, as a decrease in offspring production in freshwater cladoceran C. dubia, occurred at a concentration substantially lower than the lethal median concentration for 2,4-DANT and 2,6-DANT (Table 4.5). For TNT, 2-Am-DNT and 4-Am-DNT, sublethal effects occurred at concentrations similar to those promoting lethal effects (Table 4.5).

Reports on sublethal effects of TNT on growth or reproduction of marine fish have not been found. For freshwater fish, TNT concentration of $0.014 \mathrm{mg} \mathrm{L}^{-1}$ was reported during a 9-month TNT life cycle test as promoting a reduction in total length of the parental females after spawning was completed (Burton et al. 1993).

The toxicity of 2,4-DNT and 2,6-DNT to marine organisms was investigated by Nipper et al. (2001). Comparison of toxicity values (Tables 4.1 and 4.4) reveal that these compounds have a lower potency than TNT for the few species investigated. Neither 2,6-DNT nor its main biotransformation 
Table 4.4. Sublethal toxicity data for marine invertebrates and alga exposed to nitroaromatic MC, and comparison with lethal toxicity data, when available.

\begin{tabular}{|c|c|c|c|c|c|c|}
\hline Species & MC & \begin{tabular}{|l} 
Duration \\
(d)
\end{tabular} & $\begin{array}{l}\text { Sublethal Effect } \\
\left(\mathrm{mg} \mathrm{L}^{-1}\right)\end{array}$ & $\begin{array}{l}\text { Duration } \\
\text { (d) }\end{array}$ & \begin{tabular}{|l|} 
LC \\
$\left(\mathrm{mg} \mathrm{L}^{-1}\right)$
\end{tabular} & Reference \\
\hline \multirow{2}{*}{$\begin{array}{l}\text { Mussel } \\
\text { Mytilus } \\
\text { galloprovincialis }\end{array}$} & TNT & 2 & $\begin{array}{l}0.75 \\
\text { Embryo development } \mathrm{EC}_{50}\end{array}$ & \multirow[b]{2}{*}{4} & \multirow{2}{*}{$\begin{array}{l}19.5 \\
\text { Adult }\end{array}$} & \multirow{2}{*}{$\begin{array}{l}\text { Rosen and } \\
\text { Lotufo 2007a }\end{array}$} \\
\hline & TNT & 4 & $\begin{array}{l}6.6 \\
\text { Adult byssal thread } \\
\text { formation } \mathrm{EC}_{50}\end{array}$ & & & \\
\hline \multirow{4}{*}{$\begin{array}{l}\text { Sea Urchin } \\
\text { Arbacia punctulata }\end{array}$} & TNT & 2 & $\begin{array}{l}12 \\
\text { Embryo development } \mathrm{EC}_{50}\end{array}$ & & ND & $\begin{array}{l}\text { Nipper et al. } \\
2001\end{array}$ \\
\hline & 2,4-DNT & 2 & $\begin{array}{l}51 \\
\text { Embryo development } \mathrm{EC}_{50}\end{array}$ & & ND & $\begin{array}{l}\text { Nipper et al. } \\
2001\end{array}$ \\
\hline & 2,6-DNT & 2 & $\begin{array}{l}6.7 \\
\text { Embryo development } \mathrm{EC}_{50}\end{array}$ & & ND & $\begin{array}{l}\text { Nipper et al. } \\
2001\end{array}$ \\
\hline & 1,3,5-TNB & 2 & $\begin{array}{l}1.3 \\
\text { Embryo development } \mathrm{EC}_{50}\end{array}$ & & ND & $\begin{array}{l}\text { Nipper et al. } \\
2001\end{array}$ \\
\hline \multirow{3}{*}{$\begin{array}{l}\text { Sea Urchin } \\
\text { Lytechinus } \\
\text { variegatus }\end{array}$} & TNT & NRa & $\begin{array}{l}10 \\
\text { Embryo development } \\
\text { rerported effect }\end{array}$ & & ND & $\begin{array}{l}\text { Davenport et } \\
\text { al. } 1994\end{array}$ \\
\hline & 2,4-DNT & NR & $\begin{array}{l}10 \\
\text { Embryo development } \\
\text { rerported effect }\end{array}$ & & ND & $\begin{array}{l}\text { Davenport et } \\
\text { al. } 1994\end{array}$ \\
\hline & 2,6-DNT & NR & $\begin{array}{l}10 \\
\text { Embryo development } \\
\text { rerported effect }\end{array}$ & & ND & $\begin{array}{l}\text { Davenport et } \\
\text { al. } 1994\end{array}$ \\
\hline \multirow{4}{*}{$\begin{array}{l}\text { Polychaete } \\
\text { Dinophilus } \\
\text { gyrociliatus }\end{array}$} & TNT & 7 & $\begin{array}{l}1.1 \\
\text { Reproduction } \mathrm{EC}_{50}\end{array}$ & 7 & 5.6 & $\begin{array}{l}\text { Nipper et al. } \\
2001\end{array}$ \\
\hline & 2,4-DNT & 7 & $\begin{array}{l}5.2 \\
\text { Reproduction } \mathrm{EC}_{50}\end{array}$ & 7 & 20 & $\begin{array}{l}\text { Nipper et al. } \\
2001\end{array}$ \\
\hline & 2,6-DNT & 7 & $\begin{array}{l}2.1 \\
\text { Reproduction } \mathrm{EC}_{50}\end{array}$ & 7 & 13 & $\begin{array}{l}\text { Nipper et al. } \\
2001\end{array}$ \\
\hline & 1,3,5-TNB & 7 & $\begin{array}{l}0.4 \\
\text { Reproduction } \mathrm{EC}_{50}\end{array}$ & 7 & 1.6 & $\begin{array}{l}\text { Nipper et al. } \\
2001\end{array}$ \\
\hline \multirow{2}{*}{$\begin{array}{l}\text { Copepod } \\
\text { Schizopera knabeni }\end{array}$} & 2,6-DNT & 4 & $\begin{array}{l}55 \\
\text { Reproduction } \mathrm{EC}_{50}\end{array}$ & 4 & 65 & $\begin{array}{l}\text { Nipper et al. } \\
2005\end{array}$ \\
\hline & 2-A-6-NT & 4 & $\begin{array}{l}8 \\
\text { Reproduction } \mathrm{EC}_{50}\end{array}$ & 4 & $\begin{array}{l}\text { No lethal } \\
\text { effects at } 42 \\
\mathrm{mg} \mathrm{L}^{-1 b} \mathrm{~b}\end{array}$ & $\begin{array}{l}\text { Nipper et al. } \\
2005\end{array}$ \\
\hline \multirow{3}{*}{$\begin{array}{l}\text { Alga } \\
\text { Ulva fasciata }\end{array}$} & TNT & 4 & $\begin{array}{l}0.61 \\
\text { Germling length } \mathrm{EC}_{50}\end{array}$ & & ND & $\begin{array}{l}\text { Nipper et al. } \\
2001\end{array}$ \\
\hline & 2,4-DNT & 4 & $\begin{array}{l}1.7 \\
\text { Germling length } \mathrm{EC}_{50}\end{array}$ & & ND & $\begin{array}{l}\text { Nipper et al. } \\
2001\end{array}$ \\
\hline & 2,6-DNT & 4 & $\begin{array}{l}2.7 \\
\text { Germling length EC50 }\end{array}$ & & ND & $\begin{array}{l}\text { Nipper et al. } \\
2001\end{array}$ \\
\hline
\end{tabular}




\begin{tabular}{|l|l|l|l|l|l|l|}
\hline Species & MC & $\begin{array}{l}\text { Duration } \\
(\mathrm{d})\end{array}$ & $\begin{array}{l}\text { Sublethal Effect } \\
\left(\mathrm{mg} \mathrm{L}^{-1}\right)\end{array}$ & $\begin{array}{l}\text { Duration } \\
(\mathrm{d})\end{array}$ & $\begin{array}{l}\mathrm{LC}_{50} \\
\left(\mathrm{mg} \mathrm{L}^{-1}\right)\end{array}$ & Reference \\
\hline & $2,6-\mathrm{DNT}$ & 4 & $\begin{array}{l}4 \\
\text { Germling length EC }\end{array}$ & & ND & $\begin{array}{l}\text { Nipper et al. } \\
2005\end{array}$ \\
\cline { 2 - 7 } & $2-\mathrm{A}-6-\mathrm{NT}$ & 4 & $\begin{array}{l}20 \\
\text { Germling length EC }\end{array}$ & & ND & $\begin{array}{l}\text { Nipper et al. } \\
2005\end{array}$ \\
\cline { 2 - 7 } & $1,3,5-\mathrm{TNB}$ & 4 & $\begin{array}{l}0.03 \\
\text { Germling length EC }\end{array}$ & & ND & $\begin{array}{l}\text { Nipper et al. } \\
2001\end{array}$ \\
\hline
\end{tabular}

$\mathrm{EC}_{50}$ - median effective concentration, $\mathrm{LC}_{50}$ - median lethal concentration, ND - Not determined, a Not reported (typical duration for this assay is $2 \mathrm{~d}$ ), b Highest concentration tested.

Table 4.5. Sublethal toxicity data for freshwater cladoceran Ceriodaphnia dubia exposed to nitroaromatic $\mathrm{MC}$, and comparison with lethal toxicity data, when available. Exposur e duration was $7 \mathrm{~d}$ for all exposures.

\begin{tabular}{|c|c|c|c|}
\hline \multirow[b]{2}{*}{ MC } & Sublethal Effect & Lethal Effect & \multirow[b]{2}{*}{ Reference } \\
\hline & $\left(m g L^{-1}\right)$ & $\left(m g L^{-1}\right)$ & \\
\hline TNT & $\begin{array}{l}2.7 \\
\text { Reproduction LOEC }\end{array}$ & No effects at $2.7^{a}$ & Burton et al. 1993 \\
\hline TNT & $\begin{array}{l}3.3 \\
\text { Reproduction } \mathrm{EC}_{50}\end{array}$ & No effects at $6.0^{a}$ & Griest et al. 1998 \\
\hline 2-Am-DNT & $\begin{array}{l}3.1 \\
\text { Reproduction } \mathrm{EC}_{50}\end{array}$ & $\begin{array}{l}4.9 \\
L C_{50}\end{array}$ & Griest et al. 1998 \\
\hline 4-Am-DNT & $\begin{array}{l}.1 \\
\text { Reproduction } \mathrm{EC}_{50}\end{array}$ & $\begin{array}{l}6.6 \\
L C_{50}\end{array}$ & Griest et al. 1998 \\
\hline 2,4-DANT & $\begin{array}{l}0.05 \\
\text { Reproduction } \mathrm{EC}_{50} \\
\end{array}$ & No effects at $0.2^{a}$ & Griest et al. 1998 \\
\hline 2,6-DANT & $\begin{array}{l}0.33 \\
\text { Reproduction } \mathrm{EC}_{50}\end{array}$ & No effects at $2.0^{a}$ & Griest et al. 1998 \\
\hline 1,3,5-TNB & $\begin{array}{l}1.41 \\
\text { Reproduction } \mathrm{EC}_{50}\end{array}$ & No effects at $2.0^{a}$ & Griest et al. 1998 \\
\hline
\end{tabular}

EC50 - median effective concentration, LC50 - median lethal concentration, LOEC - Lowest observed effects concentration, a Highest concentration tested.

product, 2-A-6-NT, were particularly toxic to copepod, S. knabeni, adult female survival. The reproduction endpoint, however, indicated significantly higher toxicity of the biotransformation product (Table 4.4). The opposite happened in $U$. fasciata zoospore germination tests, where the toxicity of the parent compound, 2,6- DNT, was markedly higher than that of 2-A-6-NT (Table 4.4). Because toxicity data for only one marine fish species was found in the available literature (Nipper et al. 2001), 2,4-DNT and 2,6-DNT toxicity data for freshwater fish, an amphibian, as well as for a freshwater cladoceran, are presented in Table 4.6. For the species of fish investigated, the toxicity of 2,4-DNT and 2,6-DNT was similar among 
species and between compounds. Tadpoles were more tolerant than fish. Daphnia magna (immobilization endpoint) was more sensitive than fish (survival endpoint) and 2,4-DNT was substantially more potent than 2,6DNT to that species (Deneer et al. 1989).

Table 4.6. Toxicity data for freshwater animals exposed to DNTs.

\begin{tabular}{|c|c|c|c|c|}
\hline Species & MC & \begin{tabular}{|l} 
Duration \\
(d)
\end{tabular} & $\begin{array}{l}\text { Toxic Effect } \\
\left(\mathrm{mg} \mathrm{L}^{-1}\right)\end{array}$ & Reference \\
\hline \multirow{2}{*}{$\begin{array}{l}\text { Bullfrog tadpole } \\
\text { Rana catesbeiana }\end{array}$} & 2,4-DNT & 4 & $79.3\left(\mathrm{LC}_{50}\right)$ & Paden et al. 2011 \\
\hline & 2,6-DNT & 4 & $92.4\left(\mathrm{LC}_{50}\right)$ & Paden et al. 2011 \\
\hline \multirow{2}{*}{$\begin{array}{l}\text { Fathead minnow } \\
\text { Pimephales promelas }\end{array}$} & 2,4-DNT & $\begin{array}{l}4 \\
4\end{array}$ & $\begin{array}{l}24.3\left(\mathrm{LC}_{50}\right) \\
32.8\left(\mathrm{LC}_{50}\right)\end{array}$ & $\begin{array}{l}\text { Bailey and Spanggord } 1983 \\
\text { Broderius et al. } 2005\end{array}$ \\
\hline & 2,6-DNT & 4 & $18.5\left(\right.$ LC $\left._{50}\right)$ & Bailey and Spanggord 1983 \\
\hline \multirow{2}{*}{$\begin{array}{l}\text { Common carp } \\
\text { Cyprinus carpio }\end{array}$} & 2,4-DNT & 4 & $26.9\left(\mathrm{LC}_{50}\right)$ & Zhao et al. 1997 \\
\hline & 2,6-DNT & 4 & $20.0\left(\right.$ LC $\left._{50}\right)$ & Zhao et al. 1997 \\
\hline \begin{tabular}{|l|} 
Bluegill \\
Lepomis macrochirus
\end{tabular} & 2,4-DNT & 4 & $13.5\left(L_{50}\right)$ & Liu et al. $1983 \mathrm{~b}$ \\
\hline $\begin{array}{l}\text { Rainbow trout } \\
\text { Oncorhynchus mykiss }\end{array}$ & 2,4-DNT & 4 & $16.3\left(\mathrm{LC}_{50}\right)$ & Liu et al. $1983 \mathrm{~b}$ \\
\hline \begin{tabular}{|l|} 
Catfish \\
Ictalurus punctatus
\end{tabular} & 2,4-DNT & 4 & $24.8\left(\mathrm{LC}_{50}\right)$ & Liu et al. 1983b \\
\hline Cladoceran & 2,4-DNT & 21 & $0.6\left(E C_{50}\right)^{b}$ & Deneer et al. 1989 \\
\hline Daphnia magna & 2,6-DNT & 21 & $9.6\left(\mathrm{EC}_{50}\right)^{\mathrm{b}}$ & Deneer et al. 1989 \\
\hline
\end{tabular}

When the common carp (Cyprinus carpio) was exposed to 2,4-DNT for 7 or $15 \mathrm{~d}$, significant sublethal effects on the hepatosomatic index (at $0.5 \mathrm{mg}$ $\mathrm{L}^{-1}$ after $7 \mathrm{~d}$ ), enzyme activity (at $0.1 \mathrm{mg} \mathrm{L}^{-1}$ after $7 \mathrm{~d}$ ), energy reserves (at 1.0 $\mathrm{mg} \mathrm{L}^{-1}$ after $4 \mathrm{~d}$ ) and relative condition factor (at $0.1 \mathrm{mg} \mathrm{L}^{-1}$ after $15 \mathrm{~d}$ ) were reported ( $\mathrm{Xu}$ and Jing 2012).

\section{Toxicity of nitrophenolic MC}

When comparing the toxicity of $\mathrm{MC}$ using eight compounds of concern and marine toxicity tests with five species of marine organisms, Nipper et al. (2001), reported that tetryl, along with $1,3,5-\mathrm{TNB}$, was the most toxic compound overall (Table 4.7). It was also the most degradable compound, often being reduced to very low or below-detection levels at the end of the 
test exposure. Tetryl has not been manufactured in the United States for several decades and may no longer have any environmental relevance. In study of Nipper et al. (2001), picric acid and RDX were the least toxic chemicals tested overall. The concentrations of picric acid causing mortality to redfish and sheepshead minnows were very similar. Oysters were also tolerant to the lethal effects of picric acid (Goodfellow et al. 1983). The reduction of picric acid to picramic acid promoted substantial toxicity increase as the main breakdown products of picric acid, 2,4-DNP and picramic acid, were more toxic to copepods, oysters, and macroalga zoospores than the parent compound (Nipper et al. 2005; Goodfellow et al. 1983). The toxicity of 2,4-DNP was higher than that of picramic acid for macroalga zoospores, but both chemicals exhibited similar toxicity to copepod for reproduction (Nipper et al. 2005). The potential reasons for higher toxicity of picric acid to macroalga zoospores observed in Nipper et al. (2001) when compared to results reported in Nipper et al. (2005) were not discussed in the more recent study. The concentrations of picric acid eliciting mortality to freshwater fish (Table 4.8) were similar to concentrations reported as toxic to marine fish. Similarly, the picric acid $\mathrm{LC}_{50}$ for the marine copepod N. spinipes and the freshwater cladoceran D. magna (Table 4.8) were similar. The transformation product 2,4-DNP was more toxic than the parent compound to both marine and freshwater fish and invertebrates, most substantially so for bluegills (Tables 4.7 and 4.8).

Table 4.7. Lethal and sublethal effects concentrations for marine fish, invertebrates and alga exposed to nitrophenolic MC.

\begin{tabular}{|c|c|c|c|c|c|}
\hline \multirow[b]{2}{*}{ Species } & \multirow[b]{2}{*}{ MC } & \multirow{2}{*}{$\begin{array}{l}\text { Duration } \\
\text { (d) }\end{array}$} & Sublethal Effects & Lethal Effects & \multirow[b]{2}{*}{ Reference } \\
\hline & & & $\left(\mathrm{mg} \mathrm{L}^{-1}\right)$ & $\left(\mathrm{mg} \mathrm{L}^{-1}\right)$ & \\
\hline \multirow{2}{*}{$\begin{array}{l}\text { Redfish } \\
\text { Sciaenops } \\
\text { ocellatus }\end{array}$} & Tetryl & 2 & ND & $\begin{array}{l}1.1 \\
L C_{50}\end{array}$ & Nipper et al. 2001 \\
\hline & Picric acid & 2 & ND & $\begin{array}{l}127 \\
L_{5} C_{50}\end{array}$ & Nipper et al. 2001 \\
\hline \multirow{2}{*}{$\begin{array}{l}\text { Sheepshead } \\
\text { minnow } \\
\text { Cyprinodon } \\
\text { variegatus }\end{array}$} & Picric acid & 4 & ND & $\begin{array}{l}130 \\
L C_{50}\end{array}$ & $\begin{array}{l}\text { Heitmuller et al. } \\
1981\end{array}$ \\
\hline & 2,4-DNP & 4 & ND & $\begin{array}{l}29 \\
\mathrm{LC}_{50}\end{array}$ & $\begin{array}{l}\text { Heitmuller et al. } \\
1981\end{array}$ \\
\hline \multirow{2}{*}{$\begin{array}{l}\text { Mysid shrimp } \\
\text { Americamysis } \\
\text { bahia }\end{array}$} & Tetryl & 4 & ND & $\begin{array}{l}0.37 \\
L C_{50}\end{array}$ & Nipper et al. 2001 \\
\hline & Picric acid & 4 & ND & $\begin{array}{l}13 \\
\mathrm{LC}_{50}\end{array}$ & Nipper et al. 2001 \\
\hline
\end{tabular}




\begin{tabular}{|c|c|c|c|c|c|}
\hline \multirow[b]{2}{*}{ Species } & \multirow[b]{2}{*}{ MC } & \multirow{2}{*}{$\begin{array}{l}\text { Duration } \\
\text { (d) }\end{array}$} & Sublethal Effects & Lethal Effects & \multirow[b]{2}{*}{ Reference } \\
\hline & & & $\left(\mathrm{mg} \mathrm{L}^{-1}\right)$ & $\left(\mathrm{mg} \mathrm{L}^{-1}\right)$ & \\
\hline \multirow{2}{*}{$\begin{array}{l}\text { Sea Urchin } \\
\text { Arbacia punctulata }\end{array}$} & Tetryl & 2 & $\begin{array}{l}0.05 \\
\text { Embryo development } \\
\text { EC }_{50}\end{array}$ & ND & Nipper et al. 2001 \\
\hline & Picric acid & 2 & $\begin{array}{l}281 \\
\text { Embryo development } \\
\text { EC }_{50}\end{array}$ & ND & Nipper et al. 2001 \\
\hline \multirow{2}{*}{$\begin{array}{l}\text { Polychaete } \\
\text { Dinophilus } \\
\text { gyrociliatus }\end{array}$} & Tetryl & 7 & $\begin{array}{l}0.01 \\
\text { Reproduction } \mathrm{EC}_{50}\end{array}$ & $\begin{array}{l}0.03 \\
L C_{50}\end{array}$ & Nipper et al. 2001 \\
\hline & Picric acid & 7 & $\begin{array}{l}155 \\
\text { Reproduction } \mathrm{EC}_{50}\end{array}$ & $\begin{array}{l}265 \\
\text { LC }_{50}\end{array}$ & Nipper et al. 2001 \\
\hline \multirow{3}{*}{$\begin{array}{l}\text { Copepod } \\
\text { Schizopera } \\
\text { knabeni }\end{array}$} & Picric acid & 4 & $\begin{array}{l}60 \\
\text { Reproduction } \mathrm{EC}_{50}\end{array}$ & $\begin{array}{l}\text { No lethal effects at } \\
136 \text { a }\end{array}$ & Nipper et al. 2005 \\
\hline & $\begin{array}{l}\text { Picramic } \\
\text { acid }\end{array}$ & 4 & $\begin{array}{l}8.6 \\
\text { Reproduction } \mathrm{EC}_{50}\end{array}$ & $\begin{array}{l}\text { No lethal effects at } \\
15.4 \text { a }\end{array}$ & Nipper et al. 2005 \\
\hline & 2,4-DNP & 4 & $\begin{array}{l}9.4 \\
\text { Reproduction } \mathrm{EC}_{50}\end{array}$ & $\begin{array}{l}14 \\
\text { LC }_{50}\end{array}$ & Nipper et al. 2005 \\
\hline $\begin{array}{l}\text { Copepod } \\
\text { Nitocra spinipes }\end{array}$ & Picric acid & 4 & ND & $\begin{array}{l}92 \\
L_{5}\end{array}$ & Dave et al. 2000 \\
\hline \multirow{2}{*}{\begin{tabular}{|l} 
Oyster \\
Crassostrea \\
virginica
\end{tabular}} & Picric acid & 6 & $\begin{array}{l}28 \\
\text { Shell deposition } \mathrm{EC}_{50}\end{array}$ & $\begin{array}{l}255 \\
\mathrm{LC}_{50}\end{array}$ & $\begin{array}{l}\text { Goodfellow et al. } \\
1983\end{array}$ \\
\hline & $\begin{array}{l}\text { Picramic } \\
\text { acid }\end{array}$ & 6 & $\begin{array}{l}6 \\
\text { Shell deposition } \mathrm{EC}_{50}\end{array}$ & $\begin{array}{l}70 \\
\text { LC }_{50}\end{array}$ & $\begin{array}{l}\text { Goodfellow et al. } \\
1983\end{array}$ \\
\hline \multirow{5}{*}{$\begin{array}{l}\text { Alga } \\
\text { Ulva fasciata }\end{array}$} & Tetryl & 4 & $\begin{array}{l}0.20 \\
\text { Germling length } \mathrm{EC}_{50}\end{array}$ & ND & Nipper et al. 2001 \\
\hline & Picric acid & 4 & $\begin{array}{l}94 \\
\text { Germling length } \mathrm{EC}_{50}\end{array}$ & ND & Nipper et al. 2001 \\
\hline & Picric acid & 4 & $\begin{array}{l}472 \\
\text { Germling length } \mathrm{EC}_{50}\end{array}$ & ND & Nipper et al. 2005 \\
\hline & $\begin{array}{l}\text { Picramic } \\
\text { acid }\end{array}$ & 4 & $\begin{array}{l}88 \\
\text { Germling length } \mathrm{EC}_{50}\end{array}$ & ND & Nipper et al. 2005 \\
\hline & 2,4-DNP & 4 & $\begin{array}{l}20 \\
\text { Germling length } \mathrm{EC}_{50}\end{array}$ & ND & Nipper et al. 2005 \\
\hline
\end{tabular}

$\mathrm{EC}_{50}$ - Median effective concentration, $\mathrm{LC}_{50}$ - Median lethal concentration, ND - Not determined, a Highest concentration tested. 
Table 4.8. Lethal concentrations for freshwater fish and invertebrates exposed to nitrophenolic MC.

\begin{tabular}{|l|l|l|l|l|l|}
\hline \multirow{2}{*}{ Species } & Duration & \multicolumn{3}{|c|}{ LC50 $\left(\mathrm{mg} \mathrm{L}^{-1}\right)$} & \\
\cline { 2 - 5 } & (d) & Picric acid & $2,4-\mathrm{DNP}$ & Picramic acid & Reference \\
\hline $\begin{array}{l}\text { Rainbow trout } \\
\text { Oncorhynchus } \\
\text { mykiss }\end{array}$ & 4 & 110 & ND & 46 & $\begin{array}{l}\text { Goodfellow et al. } \\
1983\end{array}$ \\
\hline $\begin{array}{l}\text { Bluegill } \\
\text { Lepomis } \\
\text { macrochirus }\end{array}$ & 5 & 170 & 0.62 & ND & $\begin{array}{l}\text { Buccafusco et al. } \\
1981\end{array}$ \\
\hline $\begin{array}{l}\text { Cladoceran } \\
\text { Daphnia magna }\end{array}$ & 2 & 85 & 4.1 & ND & LeBlanc 1980 \\
\hline
\end{tabular}

LC50 - Median lethal concentration, ND - Not determined.

\section{Toxicity of RDX and HMX}

RDX caused decreased survival of some fish and aquatic invertebrate species at concentrations similar to those reported as acutely toxic for TNT. Other fish species and aquatic invertebrates exhibited decreased survival in exposure to concentrations approaching or exceeding $40 \mathrm{mg} \mathrm{L}^{-1}$ (Table 4.9), the approximate solubility limit for this compound in water. Lotufo et al. (2010b) and ENSR International (2005) reported similar toxicity values for RDX to sheepshead minnows (Table 4.9). Test-organisms in both studies were approximately two-week-old juvenile fish. ENSR International (2005) reported 96-h $\mathrm{LC}_{50}$ values for two species of marine fish that were lower than that for sheepshead minnows (Table 4.9). A saturated aqueous solution of RDX did not promote any effects on redfish during a 48-h embryo hatching and larval survival test (Nipper et al. 2001). The toxicity of RDX to 5 species of freshwater fish suggest they are similarly sensitive to the lethal effects of RDX as marine species (reviewed in Talmage et al. 1999 and Nipper et al. 2009; see also Muhki et al. 2005, Muhki and Patino 2008, and Warner et al. 2012).

A variety of aquatic invertebrate species, both marine and freshwater, were tolerant to the lethal effects of RDX (Peters et al. 1991; Burton et al. 1993; Dave et al. 2000; Nipper et al. 2001; ENSR International 2005; Rosen and Lotufo 2007a; Gust et al. submitted). Exposure to RDX at $28 \mathrm{mg} \mathrm{L}^{-1}$ or higher failed to elicit mortality in adult mussels, Dungeness crabs, mysid shrimp, polychaetes, amphipods and copepods, and failed to impair normal development of embryonic mussels and echinoderms (e.g., urchins) and exposure to RDX at $7.2 \mathrm{mg} \mathrm{L}^{-1}$ failed to elicit mortality of the coral Acropora formosa (Table 4.9). 
Table 4.9. Lethal and sublethal toxicity data for marine fish and invertebrates exposed to RDX or HMX.

\begin{tabular}{|c|c|c|c|c|}
\hline Species & $\mathrm{MC}$ & $\begin{array}{l}\text { Duration } \\
\text { (d) }\end{array}$ & $\begin{array}{l}\text { Effects Concentration } \\
\left(\mathrm{mg} \mathrm{L}^{-1}\right)\end{array}$ & Reference \\
\hline \multirow{3}{*}{$\begin{array}{l}\text { Sheepshead minnow } \\
\text { Cyprinodon variegatus }\end{array}$} & RDX & 10 & $9.9\left(\mathrm{LC}_{50}\right)$ & Lotufo et al. $2010 \mathrm{~b}$ \\
\hline & RDX & 4 & $9.8\left(\mathrm{LC}_{50}\right)$ & $\begin{array}{l}\text { ENSR International } \\
2005\end{array}$ \\
\hline & HMX & 5 & No lethal effects at $2^{\text {a }}$ & Lotufo et al. $2010 \mathrm{~b}$ \\
\hline $\begin{array}{l}\text { Inland silverside } \\
\text { Menidia beryllina }\end{array}$ & RDX & 4 & $7.1\left(\mathrm{LC}_{50}\right)$ & $\begin{array}{l}\text { ENSR International } \\
2005\end{array}$ \\
\hline $\begin{array}{l}\text { Speckled sand dab } \\
\text { Citharichthys stigmaeus }\end{array}$ & RDX & 4 & $2.4\left(\mathrm{LC}_{50}\right)$ & $\begin{array}{l}\text { ENSR International } \\
2005\end{array}$ \\
\hline $\begin{array}{l}\text { Redfish } \\
\text { Sciaenops ocellatus }\end{array}$ & RDX & 2 & No lethal effects at $68 \mathrm{a}, \mathrm{b}$ & Nipper et al. 2001 \\
\hline $\begin{array}{l}\text { Mussel } \\
\text { Mytilus galloprovincialis } \\
\text { (adult) }\end{array}$ & RDX & 4 & No lethal effects at 28 a & $\begin{array}{l}\text { Rosen and Lotufo } \\
2007 a\end{array}$ \\
\hline (embryo) & RDX & 2 & No developmental effects at $28^{a}$ & $\begin{array}{l}\text { Rosen and Lotufo } \\
2007 a\end{array}$ \\
\hline (embryo) & RDX & 2 & No developmental effects at $47^{\text {a }}$ & $\begin{array}{l}\text { ENSR International } \\
2005\end{array}$ \\
\hline \multirow[b]{2}{*}{ (embryo) } & $\mathrm{HMX}$ & 4 & No lethal effects at $2^{a}$ & $\begin{array}{l}\text { Rosen and Lotufo } \\
2007 a\end{array}$ \\
\hline & $\mathrm{HMX}$ & 2 & No developmental effects at 2 a & $\begin{array}{l}\text { Rosen and Lotufo } \\
2007 a\end{array}$ \\
\hline \multirow{2}{*}{$\begin{array}{l}\text { Mysid shrimp } \\
\text { Americamysis bahia }\end{array}$} & RDX & 4 & No lethal effects at $47^{a}$ & Nipper et al. 2001 \\
\hline & RDX & 4 & No lethal effects at $53^{a}$ & $\begin{array}{l}\text { ENSR International } \\
2005\end{array}$ \\
\hline $\begin{array}{l}\text { Dungeness crab } \\
\text { Cancer magister }\end{array}$ & RDX & 4 & No lethal effects at $41^{\text {a }}$ & $\begin{array}{l}\text { ENSR International } \\
2005\end{array}$ \\
\hline $\begin{array}{l}\text { Sand dollar (embryo) } \\
\text { Dendraster excentricus }\end{array}$ & RDX & 3 & No lethal effects at $41^{\text {a }}$ & $\begin{array}{l}\text { ENSR International } \\
2005\end{array}$ \\
\hline $\begin{array}{l}\text { Sea Urchin } \\
\text { Arbacia punctulata }\end{array}$ & RDX & 2 & No developmental effects at $75^{a, b}$ & Nipper et al. 2001 \\
\hline \multirow{2}{*}{$\begin{array}{l}\text { Polychaete } \\
\text { Dinophilus gyrociliatus }\end{array}$} & RDX & 7 & No lethal effects at 49 a & Nipper et al. 2001 \\
\hline & RDX & 7 & \begin{tabular}{|l}
26 \\
Reproduction (EC50)
\end{tabular} & Nipper et al. 2001 \\
\hline $\begin{array}{l}\text { Polychaete } \\
\text { Neanthes arenaceadentata }\end{array}$ & RDX & 4 & No lethal effects at 43 a & $\begin{array}{l}\text { ENSR International } \\
2005\end{array}$ \\
\hline $\begin{array}{l}\text { Copepod } \\
\text { Nitocra spinipes }\end{array}$ & RDX & 4 & No lethal effects at $36^{\text {a }}$ & Dave et al. 2000 \\
\hline $\begin{array}{l}\text { Amphipod } \\
\text { Eohaustorius estuarius }\end{array}$ & RDX & 4 & No lethal effects at 39 a & $\begin{array}{l}\text { Rosen and Lotufo, } \\
\text { unpublished data }\end{array}$ \\
\hline
\end{tabular}




\begin{tabular}{|l|l|l|l|l|}
\hline Species & MC & $\begin{array}{l}\text { Duration } \\
\text { (d) }\end{array}$ & $\begin{array}{l}\text { Effects Concentration } \\
\left(\mathrm{mg} \mathrm{L}^{-1}\right)\end{array}$ & Reference \\
\hline $\begin{array}{l}\text { Amphipod } \\
\text { Leptocheirus plumulosus }\end{array}$ & RDX & 4 & No lethal effects at 39 a & $\begin{array}{l}\text { Rosen and Lotufo, } \\
\text { unpublished }\end{array}$ \\
\hline $\begin{array}{l}\text { Coral } \\
\text { Acropora formosa }\end{array}$ & RDX & $\mathrm{g}$ & No lethal effects at 7.2 a & $\begin{array}{l}\text { Gust et al. (in } \\
\text { preparation) }\end{array}$ \\
\hline $\begin{array}{l}\text { Alga } \\
\text { Ulva fasciata }\end{array}$ & RDX & 4 & $\begin{array}{l}8.1 \\
\text { Germling length (EC }\end{array}$ & Nipper et al. 2001 \\
\hline
\end{tabular}

$\mathrm{EC}_{50}$ - Median effective concentration, a - Highest concentration tested, ${ }^{\mathrm{b}}$ Reported as target concentrations exceeding the solubility limit of $56.3 \mathrm{mg} \mathrm{L}^{-1}$ (Table 3.1).

The results of the chronic toxicity of RDX to fish and aquatic invertebrates (both freshwater and marine) are presented in Table 4.10. Surprisingly, sheepshead minnows in a 28-d exposure (ENSR International 2005) were more tolerant to RDX than in a 10-d exposure (Lotufo et al. 2010b) and Daphnia magna was substantially more tolerant to RDX in the study by ENSR International (2005) than reported in Bentley et al. (1977a).

Table 4.10. Chronic toxicity data for fish and aquatic invertebrates exposed to RDX.

\begin{tabular}{|c|c|c|c|c|c|}
\hline Species & $\begin{array}{l}\text { Duration } \\
\text { (d) }\end{array}$ & Effects & $\begin{array}{l}\text { NOEC } \\
\left(\mathrm{mg} \mathrm{L}^{-1}\right)\end{array}$ & $\begin{array}{l}\text { LOEC } \\
\left(\mathrm{mg} \mathrm{L}^{-1}\right)\end{array}$ & Reference \\
\hline \multicolumn{6}{|c|}{ Marine species } \\
\hline $\begin{array}{l}\text { Sheepshead minnow } \\
\text { Cyprinodon } \\
\text { variegatus }\end{array}$ & 28 & Survival/growth & 9.7 & 21.4 & \begin{tabular}{|l} 
ENSR \\
International \\
2005
\end{tabular} \\
\hline \multicolumn{6}{|c|}{ Freshwater species } \\
\hline \multirow{3}{*}{$\begin{array}{l}\text { Fathead minnow } \\
\text { Pimephales } \\
\text { promelas }\end{array}$} & 30 & Growth & 3.0 & 5.8 & $\begin{array}{l}\text { Bentley et al. } \\
1977 \mathrm{~b}\end{array}$ \\
\hline & 240 & Survival & 3.0 & 6.3 & $\begin{array}{l}\text { Bentley et al. } \\
\text { 1977b }\end{array}$ \\
\hline & 28 & Growth & 1.4 & 2.4 & $\begin{array}{l}\text { Burton et al. } \\
1994\end{array}$ \\
\hline $\begin{array}{l}\text { Zebrafish } \\
\text { Danio regio }\end{array}$ & 56 & Survival & 1.4 & 9.6 & $\begin{array}{l}\text { Mukhi and } \\
\text { Patino } 2008\end{array}$ \\
\hline $\begin{array}{l}\text { Cladoceran } \\
\text { Ceriodaphnia dubia }\end{array}$ & 7 & Survival/reproduction & 3.6 & 6.0 & $\begin{array}{l}\text { Peters et al. } \\
1991\end{array}$ \\
\hline \multirow{2}{*}{$\begin{array}{l}\text { Cladoceran } \\
\text { Daphnia magna }\end{array}$} & 21 & Reproduction & 2.2 & 4.8 & $\begin{array}{l}\text { Bentley et al. } \\
\text { 1977a }\end{array}$ \\
\hline & 21 & Reproduction & 41 & $\begin{array}{l}\text { No effects } \\
\text { at } 41^{\text {a }}\end{array}$ & $\begin{array}{l}\text { ENSR } \\
\text { International } \\
2005\end{array}$ \\
\hline
\end{tabular}

NOEC - No observed effect concentration, LOEC - lowest observed effect concentration, a Highest concentration tested. 
$\mathrm{RDX}$ at sublethal concentrations caused short-term negative effects on growth, but no negative effects on reproductive endpoints for zebrafish (Mukhi and Patino 2008). Maternal exposure to RDX did not affect egg fertilization and embryo hatching rates in zebrafish (Mukhi and Patino 2008). These observations indicated that $\mathrm{RDX}$ at water concentrations that do not cause significant parental mortality (up to $3.2 \mathrm{mg} \mathrm{L}^{-1}$ ) also do not have any deleterious effects on egg quality. Feeding behavior in zebrafish was affected during the first several weeks of exposure to RDX at $9.6 \mathrm{mg} \mathrm{L}^{-1}$, as fish were reported as relatively lethargic compared to fish in the control. These differences in behavior eventually disappeared towards the end of the 12-week exposure (Mukhi and Patino 2008). Incidence of vertebral deformity was observed at $1.8 \mathrm{mg} \mathrm{L}^{-1}$ for fathead minnows and at $13.8 \mathrm{mg} \mathrm{L}^{-1}$ for zebrafish after $96 \mathrm{~h}$ of exposure, approximately $2 \mathrm{x}$ lower than concentrations associated with significant lethal effects, $3.5 \mathrm{mg} \mathrm{L}^{-1}$ for fathead minnows and $27.7 \mathrm{mg} \mathrm{L}^{-1}$ for zebrafish (Warner et al. 2012). Decreased expression of collagen-coding transcripts was associated with spinal deformity and were likely involved in sensitivity to RDX. That study also found that the number and magnitude of differentially expressed transcripts increased with increasing RDX concentration for both species. Differentially expressed genes were enriched in functions related to neurological disease, oxidative-stress, acute-phase response, vitamin/mineral metabolism and skeletal/muscular disorders. Gust et al. (2011) assessed the impacts of $0.83 \mathrm{mg} \mathrm{L}^{-1}$ of RDX on fathead minnows in one-year and multigenerational bioassays. RDX had no significant effect on gonadosomatic index or condition factor assayed after up to 12 months. In addition, RDX had no significant effect on live-prey capture rates, egg production, or fertilization. Considering both marine and freshwater fish and invertebrates, the lowest sublethal effects concentration reported for RDX was $2.4 \mathrm{mg} \mathrm{L}^{-1}$ for the fathead minnow growth endpoint by Burton et al. (1994).

Exposure to HMX at concentrations reported as its maximum achieved concentration in seawater failed to elicit mortality of exposed sheepshead minnows (Lotufo et al. 2010b) and mussels (Rosen and Lotufo 2007a), both for survival and embryonic development endpoints (Table 4.6). Studies with freshwater fish (Bentely et al. 1977) demonstrated that fathead minnows were susceptible to the effects of HMX at $7 \mathrm{~d}$ post hatch, but reported a 4-d $\mathrm{LC}_{50}$ value $\left(15 \mathrm{mg} \mathrm{L}^{-1}\right)$ based on target concentrations that exceeded the solubility limit of $4.5 \mathrm{mg} \mathrm{L}^{-1}$ for that compound. All other life stages for fathead minnows as well as other fish species and invertebrates were unaffected by even the highest concentrations of HMX tested (Bentley et al. 
1977c; 1984; summarized in Talmage et al. 1999 and Nipper et al. 2009) (Table 4.11). The effect and highest no-effect concentration reported in those studies were above the reported solubility limit of HMX (Table 3.1).

Table 4.11. Toxicity data for freshwater fish and invertebrates exposed to HMX.

\begin{tabular}{|l|l|l|l|}
\hline \multirow{2}{*}{ Species } & Duration (d) & $\begin{array}{l}\text { Effects Concentration } \\
\left(\mathrm{mg} \mathrm{L}^{-1}\right)\end{array}$ & Reference \\
\hline $\begin{array}{l}\text { Fathead minnow } \\
\text { Pimephales promelas }\end{array}$ & 32 & $\begin{array}{l}15^{\mathrm{b}}\left(\mathrm{LC}_{50}\right) \\
\text { No effects on embryo } \\
\text { hatching, survival or } \\
\text { growth at 3.3 }\end{array}$ & Bentley et al. 1984 \\
\hline $\begin{array}{l}\text { Bluegill } \\
\text { Lepomis macrochirus }\end{array}$ & 4 & No lethal effects at 32 a,b & Bentley et al. 1977c \\
\hline $\begin{array}{l}\text { Rainbow trout } \\
\text { Oncorhynchus mykiss }\end{array}$ & 4 & $\begin{array}{l}\text { No lethal effects at 32 mg } \\
\text { L-1 a,b }\end{array}$ & Bentley et al. 1977c \\
\hline $\begin{array}{l}\text { Channel catfish } \\
\text { Ictalurus punctatus }\end{array}$ & 4 & No lethal effects at 32 a,b & Bentley et al. 1977c \\
\hline $\begin{array}{l}\text { Cladoceran } \\
\text { Daphnia magna }\end{array}$ & 2 & No lethal effects at 32 a,b & Bentley et al. 1977c \\
\hline $\begin{array}{l}\text { Amphipod } \\
\text { Gammarus fasciatus }\end{array}$ & 28 & $\begin{array}{l}\text { No effects on reproduction } \\
\text { at 3.9 }\end{array}$ & Bentley et al. 1984 \\
\hline $\begin{array}{l}\text { Isopod } \\
\text { Asellus militaris }\end{array}$ & 2 & No lethal effects at 32 a,b & Bentley et al. 1977c \\
\hline $\begin{array}{l}\text { Midge (larva) } \\
\text { Chironomus dilutus }\end{array}$ & 2 & No lethal effects at 32a,b & Bentley et al. 1977c \\
\hline
\end{tabular}

$\mathrm{LC}_{50}$ - median lethal concentration, a Highest concentration tested, b - Reported as target concentrations exceeding the solubility limit of $4.5 \mathrm{mg} \mathrm{L}^{-1}$ (Table 2.1).

\section{Toxicity of nitroguanadine, nitrogyclerin, nitrocellulose, PETN, and DEGDN}

The only marine receptor investigated for the toxicity of nitroguanidine (NQ) was the copepod N. spinipes (Dave et al. 2000). No lethal effect to $N$. spinipes was reported even at the highest concentration tested $\left(683 \mathrm{mg} \mathrm{L}^{-1}\right)$. Similarly, exposure of freshwater fish and aquatic invertebrates to exceedingly high concentrations of that compound also failed to result in measurable toxicity (Table 4.12). In the test with the copepod N. spinepis, the only marine receptor investigated for the toxicity of PETN, no effect was reported even at the highest concentration tested (32 $\left.\mathrm{mg} \mathrm{L}^{-1}\right)$ (Dave et al. 2000). For the freshwater cladoceran Daphnia magna, immobilization was 
Table 4.12. Lethal and sublethal toxicity data for marine and freshwater fish and invertebrates exposed to propellant related energetic compounds.

\begin{tabular}{|c|c|c|c|c|}
\hline Species & MC & $\begin{array}{l}\text { Duration } \\
\text { (d) }\end{array}$ & $\begin{array}{l}\text { Effects Concentration } \\
\left(\mathrm{mg} \mathrm{L}^{-1}\right)\end{array}$ & Reference \\
\hline \multicolumn{5}{|l|}{ Marine species } \\
\hline \multirow{2}{*}{$\begin{array}{l}\text { Copepod } \\
\text { Nitocra spinipes }\end{array}$} & NQ & 4 & No lethal effects at $683^{a}$ & Dave et al. 2000 \\
\hline & PETN & 4 & No lethal effects at 32 a & Dave et al. 2000 \\
\hline \multicolumn{5}{|l|}{ Freshwater species } \\
\hline \multirow{3}{*}{$\begin{array}{l}\text { Fathead minnow } \\
\text { Pimephales promelas }\end{array}$} & $N G$ & 4 & $2\left(\mathrm{LC}_{50}\right)$ & Bentley 1977d \\
\hline & NQ & 4 & No lethal effects at $27141 \mathrm{a}$ & van der Schalie 1985 \\
\hline & DEGDN & 4 & $491.4\left(\mathrm{LC}_{50}\right)$ & Fisher et al. 1989 \\
\hline \multirow{3}{*}{$\begin{array}{l}\text { Rainbow Trout } \\
\text { Oncorhynchus mykiss }\end{array}$} & NQ & 4 & No lethal effects at $1703^{a}$ & van der Schalie 1985 \\
\hline & $N G$ & 4 & $0.7\left(\mathrm{LC}_{50}\right)$ & Burton 1983 \\
\hline & DEGDN & 4 & $284.1\left(\mathrm{LC}_{50}\right)$ & Fisher et al. 1989 \\
\hline $\begin{array}{l}\text { Bluegill } \\
\text { Lepomis macrochirus }\end{array}$ & DEGDN & 4 & $258.0\left(\mathrm{LC}_{50}\right)$ & Fisher et al. 1989 \\
\hline $\begin{array}{l}\text { Channel catfish } \\
\text { Ictalurus punctatus }\end{array}$ & DEGDN & 4 & $278.3\left(\mathrm{LC}_{50}\right)$ & Fisher et al. 1989 \\
\hline \multirow{5}{*}{$\begin{array}{l}\text { Cladoceran } \\
\text { Daphnia magna }\end{array}$} & $N G$ & 7 & $\begin{array}{l}16 \\
\text { Lethal LOEC }\end{array}$ & Burton 1993 \\
\hline & $N G$ & 7 & $\begin{array}{l}5 \\
\text { Reproduction LOEC }\end{array}$ & Burton 1993 \\
\hline & NQ & 2 & No lethal effects at 1024 a & Dave et al. 2000 \\
\hline & PETN & 2 & No lethal effects at $49^{a}$ & Dave et al. 2000 \\
\hline & DEGDN & 2 & $90.1\left(\mathrm{LC}_{50}\right)$ & Fisher et al. 1989 \\
\hline $\begin{array}{l}\text { Midge (larva) } \\
\text { Paratanytarsus } \\
\text { parthenogeneticus }\end{array}$ & DEGDN & 2 & $159.8\left(\mathrm{LC}_{50}\right)$ & Fisher et al. 1989 \\
\hline $\begin{array}{l}\text { Mayfly } \\
\text { Hexagenia bilinata }\end{array}$ & DEGDN & 2 & $342.6\left(\mathrm{LC}_{50}\right)$ & Fisher et al. 1989 \\
\hline
\end{tabular}

a Highest concentration tested.

reported only for the highest test concentration ( $\left.49 \mathrm{mg} \mathrm{L}^{-1}\right)$ after $48 \mathrm{~h}$. No marine toxicity data was found for nitroglycerin, nitrocellulose and DEGDN. Toxicity (Table 4.12) by DEGN to freshwater fish and invertebrates only occurred at concentrations above $150 \mathrm{mg} \mathrm{L}^{-1}$ (Table 4.12) while freshwater toxicity data (Table 4.12) for nitroglycerin indicate toxic effects at a much lower range when compared to nitroguanidine, PETN and DEGDN (Table 4.12). Studies with nitrocellulose indicated no toxicity at concentrations up to $1000 \mathrm{mg} \mathrm{L}^{-1}$ when tested with several species of freshwater fish 
and invertebrates (Bentley et al. 1977c). The concentrations, however, were derived from a target concentration of an active ingredient in static toxicity tests using "a slurry of poacher pit fines" collected at an army ammunition plant, with nitrocellulose at $11.8 \%$ active ingredient. The overall lack of toxicity of nitrocellulose is likely a result of its insolubility in water.

\section{Toxicity of insenstive muntions constituents}

The military services are developing and evaluating several insensitive munitions to replace conventional organic explosives in future weapon systems. The new explosives are chemically stable enough to withstand mechanical shocks without loss of energetic performance. Among these are 3-nitro-1,2,4-trizole-5-one (NTO) as a replacement for RDX and 2,4-dinitroanisol (DNAN) as a TNT substitute (Badgujar et al. 2008). The insensitive munition formulation (IMX)-101 was recently approved as the main fill ingredient in M795 155-mm artillery munitions with over 20,00o lb manufactured (Fung et al. 2009). This relatively new insensitive munition mixture consists of DNAN, NTO, and NQ. Information on the toxicity of NTO (S\&ME 2007; Haley et al. 2009; Sayers 2009a,b) and DNAN (Kennedy et al. 2013) is limited and was not included in this summary review.

\section{Sublethal biochemical effects}

Sublethal biochemical effects can also occur due to exposure of organisms to explosive MC. Extensive investigations of biochemical-level effects on the European eel (Anguilla anguilla) were conducted by Della Torre et al. (2008a,b,c) using aqueous exposures. Their results seem to indicate an inhibitory effect of TNT on ethoxyresorufin- $O$-deethylase (EROD)dependent catalytic activities and a possible involvement of phase II enzymes as well as the reduced form of nicotinamide adenine dinucleotide phosphate (NADPH) cytochrome $\mathrm{C}$ reductase in TNT metabolism in eels (Della Torre et al. 2008a). TNT effects on the eel's neurosteroidogenic pathways was also reported (Della Torre et al. 2008b), as well as an indication that TNT is a potential competitive inhibitor of cytochrome P4501A activities. A metabolic pathway for TNT involving NADPH, NADPH cytochrome $\mathrm{C}$ reductase and phase II enzymes is also suggested (Della Torre et al. 2008c).

Significant inhibition of EROD activity was also observed in juvenile rainbow trout (Onchorhynchus mykiss) dosed intraperitoneally with TNT at 
different concentrations (Ek et al. 2003). In a similar study, trout treated with TNT had increased glutathione $S$-transferase and glutathione reductase activities, and a decreased percentage of oxidized glutathione compared to control fish (Ek et al. 2005), indicating that TNT oxidizes macromolecules and activates antioxidant defense systems. The compounds TNT, 2-Am-DNT and 4-Am-DNT were found in the hydrolyzed bile of the TNT-treated trout, indicating that this fish species can detoxify and excrete TNT. The presence of TNT and its transformation products in the bile may be suitable as a direct marker of TNT exposure in fish at contaminated sites (Ek et al. 2005).

Gust et al. (in preparation) has demonstrated increased expression of cytochrome $\mathrm{P} 450$ in coral (Acropora formosa) exposed to RDX as a mechanism to detoxify the chemical insult. In addition, potential insult to coral health was evidenced by the significant increase of coral mucocyte densities at the maximum RDX exposure $\left(7.2 \mathrm{mg} \mathrm{L}^{-1}\right)$, but the zooxanthellae tissue densities were not affected.

Advances in molecular biology are extending the use of biomarkers to the level of the genes, which, when coupled with bioinformatics, are progressing towards the development of powerful new tools to ecotoxicologists. Environmental genomics (using gene expression profiles after exposure to toxicants, also called toxicogenomics) allow insight into modes of action and may be used to assess the causal agents and potential toxicity (GarciaReyero et al. 2009). Recent research aims the development of robust methods for using gene expression analysis for identifying gene expression biomarkers of chemical exposure that can be further used to monitor specific chemical exposures in the environment (Garcia-Reyero et al. 2009, 2012).

Gust et al. (2011) conducted a comprehensive investigation of the effects that RDX exposure elicited on transcript expression in fathead minnow brain tissue. Significant mortality occurred a $10 \mathrm{mg} \mathrm{L}^{-1}$ after 10 days of exposure to spiked water. Conversely, microarray data indicated significant changes in transcript expression at concentrations as low as $0.625 \mathrm{mg} \mathrm{L}^{-1}$. Individual gene functions affected by RDX exposures indicated changes in metabolic processes involved in: oxygen transport, neurological function, calcium binding/signaling, energy metabolism, cell growth/division, oxidative stress and ubiquitination. 
Using a custom complementary DNA (cDNA) microarray for Daphnia magna, Garcia-Reyero et al. (2009) identified distinct expression fingerprints in response to exposure at sublethal concentrations of MC. RDX exhibited a robust gene expression pattern affecting diverse pathways involved in exoskeletal maintenance such as protein catabolism, cell structure, and cellular transport. An induction of oxidative stress response genes was seen after exposure to 1,3,5-TNB, DNB, and TNT. Both 4-AmDNT and 2,4-DNT affected genes (e.g., vitellogenin) directly involved in reproduction and development. TNT caused up-regulation of ferritin, indicating possible interference with iron metabolism or oxidative stress. In a follow-up study, Garcia-Reyero et al. (2012) have demonstrated that, to a limited extent, it is possible to predict gene expression changes in the $D$. magna given the chemical mixture to which it has been exposed.

\section{Relationship between bioaccumulation of MC and toxicity}

This subsection describes the relationship between bioaccumulation and toxic effects. Section 5 discusses bioaccumulation of TNT, RDX, and HMX in marine fish and aquatic invertebrates and reports uptake rates, elimination rates, half lives, and bioconcentration factors for those compounds.

Three of the studies cited in this chapter (Rosen and Lotufo 2007a; Lotufo et al. 2010a, and Rosen and Lotufo, unpublished data) measured the whole body tissue concentration (or whole body burden) of toxicity test organisms. Exposure to TNT resulted in the bioaccumulation of the parent compound, as well as of the transformation products 2-Am-DNT and 4-Am-DNT in sheepshead minnows (Lotufo et al. 2010a), adult mussels (Rosen and Lotufo 2007a) and amphipods (Rosen and Lotufo, unpublished). The sum concentration of 2-Am-DNT and 4-Am-DNT formed by biotransformation exceeded that of TNT in adult mussels and in the amphipod L. plumulosus. Exposure to 2-Am-DNT and 2,4-DANT resulted in detectable accumulation of those compounds in fish (Lotufo et al. 2010a) and no apparent biotransformation. However, fish exposed to 1,3,5-TNB failed to bioaccumulate parent and transformation products above detection levels (Lotufo et al. 2010a). Aqueous exposure to RDX and HMX resulted in measurable bioaccumulation of parent compounds in fish (Lotufo et al. 2010b; Muhki and Patino 2008) and in invertebrates and no apparent biotransformation (Rosen and Lotufo 2007a, 2007b; Rosen and Lotufo, unpublished).

When the whole body residue can be linked to a specific biological effect, the body residue is termed the critical body residue (CBR) (McCarty and 
Mackay, 1993). Typically, tissue residue concentrations are more directly relatable to toxic effects than water-based concentrations and provide an integrated assessment of the exposure an organism receives over time and space (Meador et al. 2006).

Critical residues associated with significant mortality of organisms exposed to MC are presented in Table 4.13 as median lethal residues $\left(\mathrm{LR}_{50}\right)$. For exposures that did not result in significant mortality, the body residues in organisms surviving the highest exposure concentrations are reported as no-observed-effect residue (NOER).

Table 4.13. Body residue toxicity metrics for freshwater and marine invertebrates exposed to nitroaromatic MC.

\begin{tabular}{|c|c|c|c|c|c|c|}
\hline \multirow[b]{2}{*}{ Species } & \multirow[b]{2}{*}{ Habitat } & \multirow[b]{2}{*}{ Endpoint } & \multicolumn{2}{|c|}{ Body Residue } & \multirow{2}{*}{$\begin{array}{l}\text { Lethal } \\
\text { Concentration } \\
\left(\mathrm{mg} \mathrm{L}^{-1}\right)^{a}\end{array}$} & \multirow[b]{2}{*}{ Reference } \\
\hline & & & $\begin{array}{l}\text { nmol g-1 } \\
\text { wet wt }\end{array}$ & $\begin{array}{l}\text { mg kg-1 } \\
\text { wet wt }\end{array}$ & & \\
\hline \multicolumn{7}{|c|}{ SumTNT } \\
\hline $\begin{array}{l}\text { Sheepshead minnow } \\
\text { Cyprinodon } \\
\text { variegatus }\end{array}$ & Marine & 10-d LR50 & 26 & 5.4 & $\begin{array}{l}2.3 \\
10-d L_{50}\end{array}$ & $\begin{array}{l}\text { Lotufo et al. } \\
2010 a\end{array}$ \\
\hline $\begin{array}{l}\text { Fathead minnow } \\
\text { Pimephales promelas }\end{array}$ & Freshwater & $\begin{array}{l}\text { mean residue } \\
\text { associated with } \\
10-d 50 \% \\
\text { mortality }\end{array}$ & 15 & 3.4 & $\begin{array}{l}2.2 \\
10-d L_{50}\end{array}$ & Yoo at al. 2006 \\
\hline $\begin{array}{l}\text { Mussel (adult) } \\
\text { Mytilus } \\
\text { galloprovincialis }\end{array}$ & Marine & 4-d LR50 & 51 & 14.0 & $\begin{array}{l}19.5 \\
4-d \text { LC50 }\end{array}$ & $\begin{array}{l}\text { Rosen and } \\
\text { Lotufo } 2007 a\end{array}$ \\
\hline $\begin{array}{l}\text { Amphipod } \\
\text { Eohaustorious } \\
\text { estuarius }\end{array}$ & Marine & 4-d LR50 & 162 & 36.8 & $\begin{array}{l}4.5 \\
4-d \text { LC50 }\end{array}$ & $\begin{array}{l}\text { Rosen and } \\
\text { Lotufo, } \\
\text { unpublished }\end{array}$ \\
\hline $\begin{array}{l}\text { Amphipod } \\
\text { Leptocheirus } \\
\text { plumulosus }\end{array}$ & Marine & 4-d LR50 & 77 & 17.5 & $\begin{array}{l}3.6 \\
4-d L_{50}\end{array}$ & $\begin{array}{l}\text { Rosen and } \\
\text { Lotufo, } \\
\text { unpublished }\end{array}$ \\
\hline $\begin{array}{l}\text { Oligochaete } \\
\text { Tubifex tubifex }\end{array}$ & Freshwater & 4-d LR50 & 172 & 39.0 & $\begin{array}{l}7.7 \\
4-d L_{50}\end{array}$ & $\begin{array}{l}\text { Conder et al. } \\
2004 c\end{array}$ \\
\hline $\begin{array}{l}\text { Midge (larva) } \\
\text { Chironomus dilutus }\end{array}$ & Freshwater & 4-d LR50 & 118 & 8.9 & $\begin{array}{l}1.9 \\
4-d \text { LC50 }\end{array}$ & $\begin{array}{l}\text { Conder et al. } \\
2004 c\end{array}$ \\
\hline \multicolumn{7}{|c|}{ 2-Am-DNT } \\
\hline $\begin{array}{l}\text { Cyprinodon } \\
\text { variegatus }\end{array}$ & Marine & 5-d LR50 & 275 & 54.2 & $\begin{array}{l}8.6 \\
5-d \text { LC50 }\end{array}$ & $\begin{array}{l}\text { Lotufo et al. } \\
2010 a\end{array}$ \\
\hline \multicolumn{7}{|c|}{ DANT } \\
\hline $\begin{array}{l}\text { Cyprinodon } \\
\text { variegatus }\end{array}$ & Marine & 5-d NOER & 284 & 47.4 & $\begin{array}{l}\text { No lethal effects at } \\
50.3 \mathrm{mg} \mathrm{L}^{-1} \mathrm{~b}\end{array}$ & $\begin{array}{l}\text { Lotufo et al. } \\
2010 a\end{array}$ \\
\hline
\end{tabular}




\begin{tabular}{|c|c|c|c|c|c|c|}
\hline \multirow[b]{2}{*}{ Species } & \multirow[b]{2}{*}{ Habitat } & \multirow[b]{2}{*}{ Endpoint } & \multicolumn{2}{|c|}{ Body Residue } & \multirow{2}{*}{$\begin{array}{l}\text { Lethal } \\
\text { Concentration } \\
\left(\mathrm{mg} \mathrm{L}^{-1}\right)^{\mathrm{a}}\end{array}$} & \multirow[b]{2}{*}{ Reference } \\
\hline & & & $\begin{array}{l}\text { nmol g-1 } \\
\text { wet wt }\end{array}$ & $\begin{array}{l}\mathrm{mg} \mathrm{kg}^{-1} \\
\text { wet wt }\end{array}$ & & \\
\hline \multicolumn{7}{|c|}{ RDX } \\
\hline $\begin{array}{l}\text { Cyprinodon } \\
\text { variegatus }\end{array}$ & Marine & 10-d LR50 & 135 & 30.0 & $\begin{array}{l}9.9 \\
10-\mathrm{d} \mathrm{LC}\end{array}$ & $\begin{array}{l}\text { Lotufo et al. } \\
2010 \mathrm{~b}\end{array}$ \\
\hline $\begin{array}{l}\text { Zebrafish } \\
\text { Danio regio }\end{array}$ & Freshwater & $\begin{array}{l}\text { Mortality (33\% } \\
\text { after } 28 \mathrm{~d} \text { ) }\end{array}$ & 40 & 9 & $\begin{array}{l}9.6 \\
33 \% \text { mortality after } \\
28 d\end{array}$ & $\begin{array}{l}\text { Mukhi and } \\
\text { Patino } 2008\end{array}$ \\
\hline $\begin{array}{l}\text { Mytilus } \\
\text { galloprovincialis }\end{array}$ & Marine & 4-d NOER & 86 & 19.6 & $\begin{array}{l}\text { No lethal effects at } \\
28 \mathrm{mg} \mathrm{L}^{-1 \mathrm{~b}}\end{array}$ & $\begin{array}{l}\text { Rosen and } \\
\text { Lotufo 2007a }\end{array}$ \\
\hline $\begin{array}{l}\text { Eohaustorious } \\
\text { estuarius }\end{array}$ & Marine & 4-d NOER & 268 & 59.5 & $\begin{array}{l}\text { No lethal effects at } \\
39 \mathrm{mg} \mathrm{L}^{-1 \mathrm{~b}}\end{array}$ & $\begin{array}{l}\text { Rosen and } \\
\text { Lotufo, } \\
\text { unpublished }\end{array}$ \\
\hline $\begin{array}{l}\text { Leptocheirus } \\
\text { plumulosus }\end{array}$ & Marine & 4-d NOER & 191 & 42.4 & $\begin{array}{l}\text { No lethal effects at } \\
39 \mathrm{mg} \mathrm{L}^{-1 \mathrm{~b}}\end{array}$ & $\begin{array}{l}\text { Rosen and } \\
\text { Lotufo, } \\
\text { unpublished }\end{array}$ \\
\hline \multicolumn{7}{|c|}{ HMX } \\
\hline $\begin{array}{l}\text { Cyprinodon } \\
\text { variegatus }\end{array}$ & Marine & 10-d NOER & 14 & 4.1 & $\begin{array}{l}\text { No lethal effects at } \\
2 \mathrm{mg} \mathrm{L}-1 \mathrm{~b}\end{array}$ & $\begin{array}{l}\text { Lotufo et al. } \\
2010 \mathrm{~b}\end{array}$ \\
\hline $\begin{array}{l}\text { Mytilus } \\
\text { galloprovincialis }\end{array}$ & Marine & 4-d NOER & 3 & 0.9 & $\begin{array}{l}\text { No lethal effects at } \\
2 \mathrm{mg} \mathrm{L^{-1 } \mathrm { b }}\end{array}$ & $\begin{array}{l}\text { Rosen and } \\
\text { Lotufo 2007a }\end{array}$ \\
\hline
\end{tabular}

$\mathrm{LR}_{50}$ - Median lethal residue, NOER - No-observed-effect residue, a Concentration in the exposure water associated with observed effects, b Highest concentration tested.

For SumTNT, the CBR was similar for sheepshead and fathead minnows (Lotufo et al. 2010b; Yoo et al. 2006), suggesting similar sensitivity among fish species. For invertebrates, the SumTNT CBR was lowest for mussels and highest for the amphipod E. estuarius. The CBRs from SumTNT fall within a range that is substantially lower than the range considered typical for compounds causing mortality by general narcosis (McCarty and Mackay, 1993), suggesting that lethal effects of TNT are associated with specific modes of action.

The lowest CBR for RDX was reported for zebrafish exposed to $9.6 \mathrm{mg} \mathrm{L}^{-1}$ for $28 \mathrm{~d}$ by Mukhi and Patino (2008), who reported 33\% mortality of fish exposed for $28 \mathrm{~d}$. A higher CBR was obtained for sheepshead minnows exposed to $9.9 \mathrm{mg} \mathrm{L}^{-1}$ for $10 \mathrm{~d}$ by Lotufo et al. (2010a). Water-only exposure of adult mussels and amphipods to even higher concentrations of RDX in water failed to result in significant decrease in survival (Rosen and Lotufo 2007a; Rosen and Lotufo in preparation). Body residues were measured in studies exposing sheepshead minnows (Lotufo et al. 2010a) or mussels 
(Rosen and Lotufo 2007a) to concentrations of HMX in water approaching its solubility limit. CBRs could not be determinded in those studies due to lack of significant mortality.

If the CBR of a chemical is known, or can be estimated, and confidence about its application to the species of concern exists, the CBR approach can be directly used for assessment of potential risk to the population using organisms collected in the field. When the tissue concentration of a given chemical is well below its CBR (e.g., by order of magnitude) in fieldcollected specimens, that chemical would not be considered a toxicity driver. An example of the use of this approach in field assessment is provided in Redman et al. (2012). Most MC are efficiently biotransformed and eliminated by fish and aquatic invertebrates (Section 6). The exposure concentration of MC may vary over time depending on the source of contamination to the water column. Therefore, aquatic organisms, especially mobile species, may have body residue that vary widely overtime, complicating the use of the CBR approach in field evaluations. 


\section{Toxicity of Munitions Constituents to Aquatic Invertebrates and Fish in Sediment Exposures}

This section summarizes the available data on the toxicity of sedimentassociated explosives to marine and freshwater fish and invertebrates. Laboratory studies used sediment amended with explosives which were used in toxicity tests using whole-sediment or extracted porewater.

\section{Overview}

Major explosive contaminants such as 2,4,6-trinitrotoluene TNT, 1,3,5TNB, RDX, HMX, and tetryl and their transformation products were detected in freshwater sediment samples collected from military installations at concentrations ranging from low (e.g., less than $6.7 \mathrm{mg} \mathrm{kg}^{-1}$ ) to high percent levels (up to 711,000 $\mathrm{mg} \mathrm{kg}^{-1}$ ) (Talmage et al. 1999). Those extremely high concentrations ("hot spots") are indicative of large amounts of non-dissolved and non-sorbed explosives in some contaminated sites. Low concentrations ( $\left.<0.003 \mathrm{mg} \mathrm{kg}^{-1}\right)$ of 2,6-dinitrotoluene (2,6-DNT), tetryl, and 2,4,6-trinitrophenol (picric acid) acid were detected in marine sediments near a naval facility in Ostrich Bay, Puget Sound, WA (Pascoe et al. 2010). Field experiments using cleaved shells containing TNT demonstrated that leakage from breached munitions can serve as a source of sediment contamination (Dave 2003).

The fate of explosive compounds in soils has been well characterized (Pennington and Brannon 2002, Monteil-Rivera et al. 2009). In contrast, fewer studies addressed the fate of explosives and related compounds in sediments, despite the potential ecological risk associated with their presence in this compartment of aquatic environments. Recent studies characterizing the processes that occur following the initial contact of explosives and related compounds with sediments revealed rapid rates of transformation for a variety of nitroaromatic compounds (Elovitz and Weber 1999; Lotufo et al. 2010c; Pennington et al. 2011).

Compared to investigations addressing the soil ecotoxicity of $\mathrm{MC}$ (reviewed by Kuperman et al. 2009), few studies addressed the toxicity of sediment-associated MC to marine invertebrates (Green et al. 1999; Dave 
et al. 2000; Lotufo et al. 2001; Nipper et al. 2002; Nipper et al. 2005; Rosen and Lotufo 2005), as well to freshwater invertebrates (Steevens et al. 2002; Conder et al. 2004a; Lotufo and Farrar 2005), as reviewed in Lotufo et al. (2009a). Infaunal marine invertebrates used in those investigations burrow in and ingest sediment, and are, therefore, potentially exposed to sediment contaminants via sediment and food particles, interstitial (pore) water, and overlying water. Bottom-dwelling fish come into contact with sediment-associated contaminants through direct uptake from benthic prey, directly from sediment bed particles through ingestion or dermal contact, and from the overlying water via suspended sediment particles, or the dissolved phase. The evaluation of toxicity of MC to fish resulting from exposure to contaminated sediment was also investigated (Lotufo et al. 2010c).

\section{Fate of nitroaromatic MC amended to sediment for toxicity testing}

Fate kinetics and mass balance studies conclusively demonstrate that the fate of TNT in wet sediment is dependent on a combination of reductive transformation and sorption processes (Elovitz and Weber 1999; Pennington et al. 2011).

When TNT was spiked into marine or freshwater sediments for toxicity and bioavailability investigations, aminodinitrotoluenes (Am-DNTs) and diaminonitrotoluenes (DANTs) were detected following the short $(4 \mathrm{~h})$ postspiking mixing periods, with transformation continuing during storage or toxicological testing for both marine (Rosen and Lotufo 2005; Lotufo et al. 2010c) and freshwater sediments (Steevens et al. 2002; Conder et al. 2004a; Lotufo and Farrar 2005). An inverse relationship between the TNT spiking concentration and the transformation rate has been reported on a percentage basis (Rosen and Lotufo 2005; Lotufo et al. 2010c) (Figures 5.1 and 5.2). That is, the lower the concentration of TNT added to sediment, the higher the observance of transformation to Am-DNTs, and to their daughter product, DANTs on a percent of total TNT basis. The rate of transformation appears to decrease with increasing spiking concentration, as observed for sediment in Lotufo et al. (2010c) (Figure 5.2). Decreasing TNT transformation at high spiking concentrations likely resulted from increasing saturation of binding sites in the sediment or soil particles and inhibition of microbial activity. 


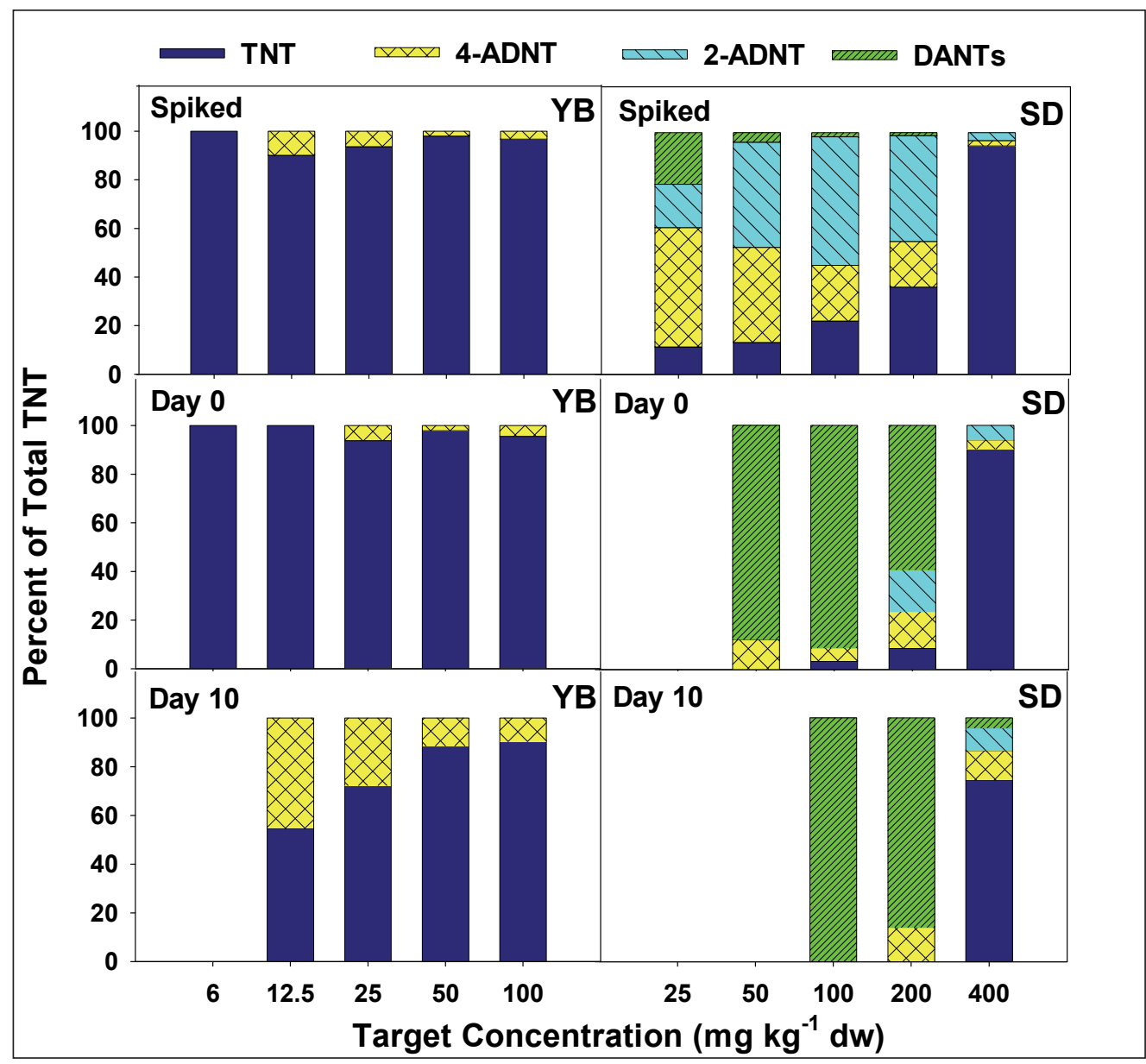

Figure 5.1. Percent of total concentration in sediment samples corresponding to the concentration of trinitrotoluene (TNT), 2-aminodinitrotoluene (2-Am-DNT), 4aminodinitrotoluene (4-Am-DNT), and diaminonitrotoluenes (DANTs) in solvent extracts of ${ }^{14} \mathrm{C}-\mathrm{TNT}$-spiked Yaquina Bay (YB) and San Diego Bay (SD) toxicity test sediments (from Rosen and Lotufo 2005). Absence of bars indicates neither that TNT nor its transformation products were detectable.

The transformation of TNT to aminated products was substantially lower in marine coarse-grained than in fine-grained sediment when both were spiked with the same initial organic-carbon-normalized concentrations (Rosen and Lotufo 2005). When sand with non-detectable total organic carbon (TOC) was spiked with TNT, only a small degree of transformation to Am-DNTs occurred over time, while spiking fine-grained sediment (TOC $=1.1 \%$ ) with TNT resulted in a large degree of transformation (Figure 5.2). The stimulatory effect of enhanced sediment TOC on the transformation of TNT in water overlying the contaminated sediment was demonstrated by Harrison and Vane (2010). 


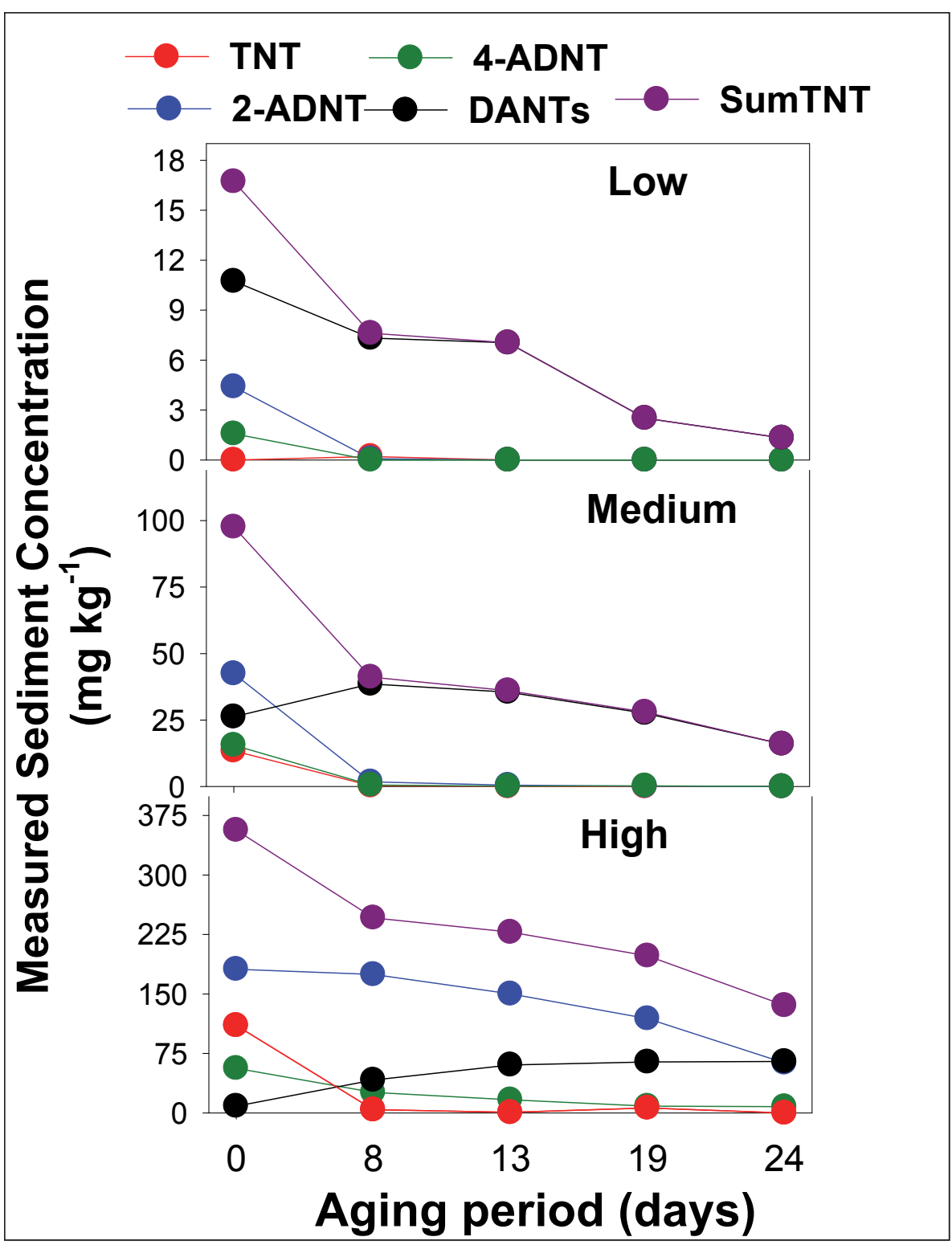

Figure 5.2. TNT and transformation product concentrations over time in finegrained marine sediment (1.1\% total organic carbon) (from Lotufo et al. 2010c).

When TNT was spiked into marine sediments, as the contact time with sediment ("aging period") increased, the proportion of solvent-extractable TNT in the sediment decreased (Steevens et al. 2002, Conder et al. 2004a; reviewed in Lotufo et al. 2009a). Such a decrease of solvent-extractable TNT and TNT transformation products in marine sediments following spiking was attributed to covalent binding to sediment organic matter (Pennington et al. 2011) and has been extensively reported for soils (Eriksson et al. 2004, Thorn et al. 2004, Singh et al. 2010) and freshwater sediments (Elovitz and Weber 1999). A description of the different types of mechanisms 
responsible for binding of TNT and its degradation products to soil organic matter is provided in Singh et al. (2010).

The fraction of solvent-resistant transformation products of TNT, likely associated with the sediment through covalent binding to sediment particles was higher in spiked fine-grained sediment compared to sandy sediment (Rosen and Lotufo 2005) (Figure 5.3).

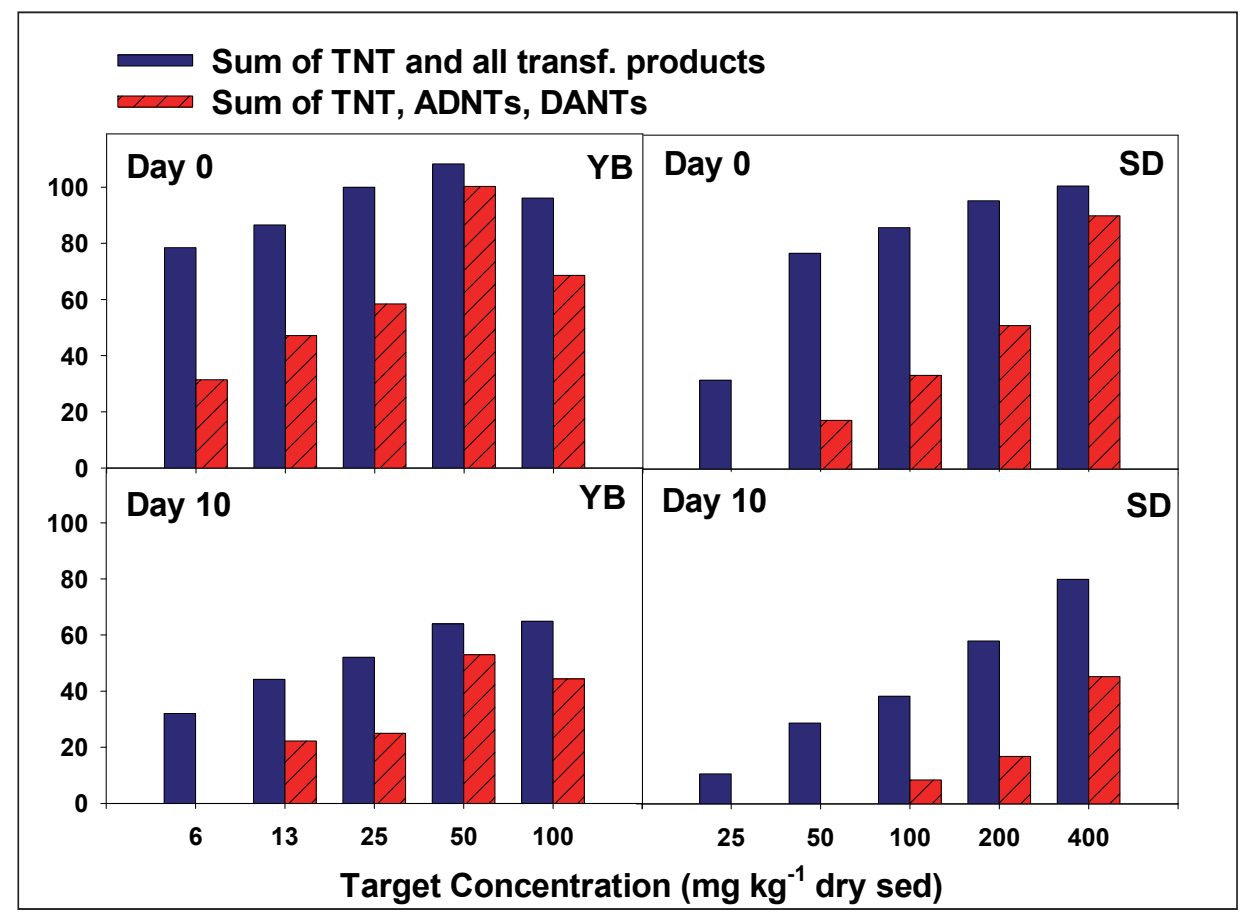

Figure 5.3. Percent of total radioactivity in sediment corresponding to the sum of solvent-extracted TNT and all its transformation products, including unknown compounds, as determined by radioactivity (blue), and to the sum of solventextracted TNT, Am-DNTs and DANTs, as determined by chemical analysis (red) in sandy sediment (Yaquina Bay, YB) and fine-grained sediment (San Diego Bay, SD) at initiation (Day 0) and termination (Day 10) of a toxicity test (from Rosen and Lotufo 2005). Absence of bars indicates neither that TNT nor its transformation products were detectable.

The measured sum concentration of TNT and its major transformation products, Am-DNTs and DANTs (SumTNT), in marine and freshwater sediments spiked with TNT was typically lower than the target (or nominal) concentration following a short mixing period (up to $4 \mathrm{~h}$ ) (Table 5.1), indicating that rapid transformation of spiked TNT to compounds that sorb strongly to sediment and resisted organic solvent extraction occurs in a variety of sediment types. 
Table 5.1. Target concentrations and concentrations measured after spiking with TNT and mixing for sediments of different types and total organic carbon (TOC) content used in toxicity tests.

\begin{tabular}{|c|c|c|c|c|c|c|c|}
\hline \multirow{2}{*}{$\begin{array}{l}\text { Sediment type } \\
\text { and source }\end{array}$} & \multirow{2}{*}{$\begin{array}{l}\text { TOC } \\
\text { (\%) }\end{array}$} & \multicolumn{2}{|c|}{$\begin{array}{l}\text { SumTNT Concentration } \\
\left(\mathrm{mg} \mathrm{kg}^{-1}\right)\end{array}$} & \multirow{2}{*}{$\begin{array}{l}\text { Ratio } \\
\text { Meas/Tgt }\end{array}$} & \multirow{2}{*}{$\begin{array}{l}\text { Mixing } \\
\text { period }(h)\end{array}$} & \multirow{2}{*}{$\begin{array}{l}\text { Moisture } \\
\text { conditiona }\end{array}$} & \multirow[b]{2}{*}{ Reference } \\
\hline & & Target & Measured & & & & \\
\hline \multirow{10}{*}{$\begin{array}{l}\text { Fine-grained } \\
\text { Freshwater }\end{array}$} & 0.65 & 12.5 & $<0.1^{b}$ & 0.004 & 0.5 & Dry & $\begin{array}{l}\text { Steevens et } \\
\text { al. } 2002\end{array}$ \\
\hline & 0.65 & 25 & $<0.1^{b}$ & 0.002 & 0.5 & Dry & $\begin{array}{l}\text { Steevens et } \\
\text { al. } 2002\end{array}$ \\
\hline & 0.65 & 50 & 0.1 & 0.002 & 0.5 & Dry & $\begin{array}{l}\text { Steevens et } \\
\text { al. } 2002\end{array}$ \\
\hline & 0.65 & 100 & 4.3 & 0.04 & 0.5 & Dry & $\begin{array}{l}\text { Steevens et } \\
\text { al. } 2002\end{array}$ \\
\hline & 0.65 & 200 & 66.5 & 0.33 & 0.5 & Dry & $\begin{array}{l}\text { Steevens et } \\
\text { al. } 2002\end{array}$ \\
\hline & 0.65 & 400 & 153.3 & 0.38 & 0.5 & Dry & $\begin{array}{l}\text { Steevens et } \\
\text { al. } 2002\end{array}$ \\
\hline & 0.65 & 127 & 28 & 0.22 & 1 & Wet & $\begin{array}{l}\text { Lotufo and } \\
\text { Farrar } 2005\end{array}$ \\
\hline & 0.65 & 238 & 53 & 0.22 & 1 & Wet & $\begin{array}{l}\text { Lotufo and } \\
\text { Farrar } 2005\end{array}$ \\
\hline & 0.65 & 605 & 135 & 0.22 & 1 & Wet & $\begin{array}{l}\text { Lotufo and } \\
\text { Farrar } 2005\end{array}$ \\
\hline & 0.65 & 1365 & 304 & 0.22 & 1 & Wet & $\begin{array}{l}\text { Lotufo and } \\
\text { Farrar } 2005\end{array}$ \\
\hline \multirow{5}{*}{$\begin{array}{l}\text { Fine-grained } \\
\text { Marine }\end{array}$} & 0.36 & 25 & 23 & 0.94 & 4 & Wet & $\begin{array}{l}\text { Rosen and } \\
\text { Lotufo } 2005\end{array}$ \\
\hline & 0.36 & 50 & 52 & 1.03 & 4 & Wet & $\begin{array}{l}\text { Rosen and } \\
\text { Lotufo } 2005\end{array}$ \\
\hline & 0.36 & 100 & 92 & 0.92 & 4 & Wet & $\begin{array}{l}\text { Rosen and } \\
\text { Lotufo } 2005\end{array}$ \\
\hline & 0.36 & 200 & 148 & 0.74 & 4 & Wet & $\begin{array}{l}\text { Rosen and } \\
\text { Lotufo } 2005\end{array}$ \\
\hline & 0.36 & 400 & 350 & 0.87 & 4 & Wet & $\begin{array}{l}\text { Rosen and } \\
\text { Lotufo } 2005\end{array}$ \\
\hline \multirow{5}{*}{$\begin{array}{l}\text { Sandy } \\
\text { Marine }\end{array}$} & 0.09 & 6 & 3 & 0.46 & 4 & Wet & $\begin{array}{l}\text { Rosen and } \\
\text { Lotufo } 2005\end{array}$ \\
\hline & 0.09 & 12.5 & 76 & 0.62 & 4 & Wet & $\begin{array}{l}\text { Rosen and } \\
\text { Lotufo } 2005\end{array}$ \\
\hline & 0.09 & 25 & 18 & 0.72 & 4 & Wet & $\begin{array}{l}\text { Rosen and } \\
\text { Lotufo } 2005\end{array}$ \\
\hline & 0.09 & 50 & 37 & 0.73 & 4 & Wet & $\begin{array}{l}\text { Rosen and } \\
\text { Lotufo } 2005\end{array}$ \\
\hline & 0.09 & 100 & 63 & 0.63 & 4 & Wet & $\begin{array}{l}\text { Rosen and } \\
\text { Lotufo } 2005\end{array}$ \\
\hline
\end{tabular}

a Analysis followed solvent extraction of dry or wet substrate, b Numerical value represents the detection limit. Assumed as $0.05 \mathrm{mg} \mathrm{kg}^{-1}$ for calculating the measured/target ratio. 
Exposure chambers containing sediment amended with TNT and uncontaminated overlying water were used for toxicity testing (Rosen and Lotufo 2005). A substantial portion (up to $50 \%$ ) of the ${ }^{14} \mathrm{C}$ from the radiolabeled TNT originally associated with the sediment particles was present in the porewater and in the non-renewed overlying water (contained the greater fraction of the $\mathrm{C}^{14}$ ) at the end of the $10 \mathrm{~d}$ exposures (Figure 5.4). Such high partitioning was expected given the reported relatively high aqueous solubility of TNT and its transformation products (Table 3.1). Renewal of overlying waters is typically performed during chronic sediment exposures using benthic invertebrates to maintain adequate water quality, especially when feeding is required. However, exchange of overlying water during invertebrate exposures to TNT-spiked sediment is expected to rapidly deplete contaminants from the exposure system. Therefore, it is recommended that toxicity evaluations of nitroaromatic compounds spiked to sediments should use exposure conditions (e.g., duration, water quality requirements) that do not require water changes during the experimental period (Conder et al. 2004a).

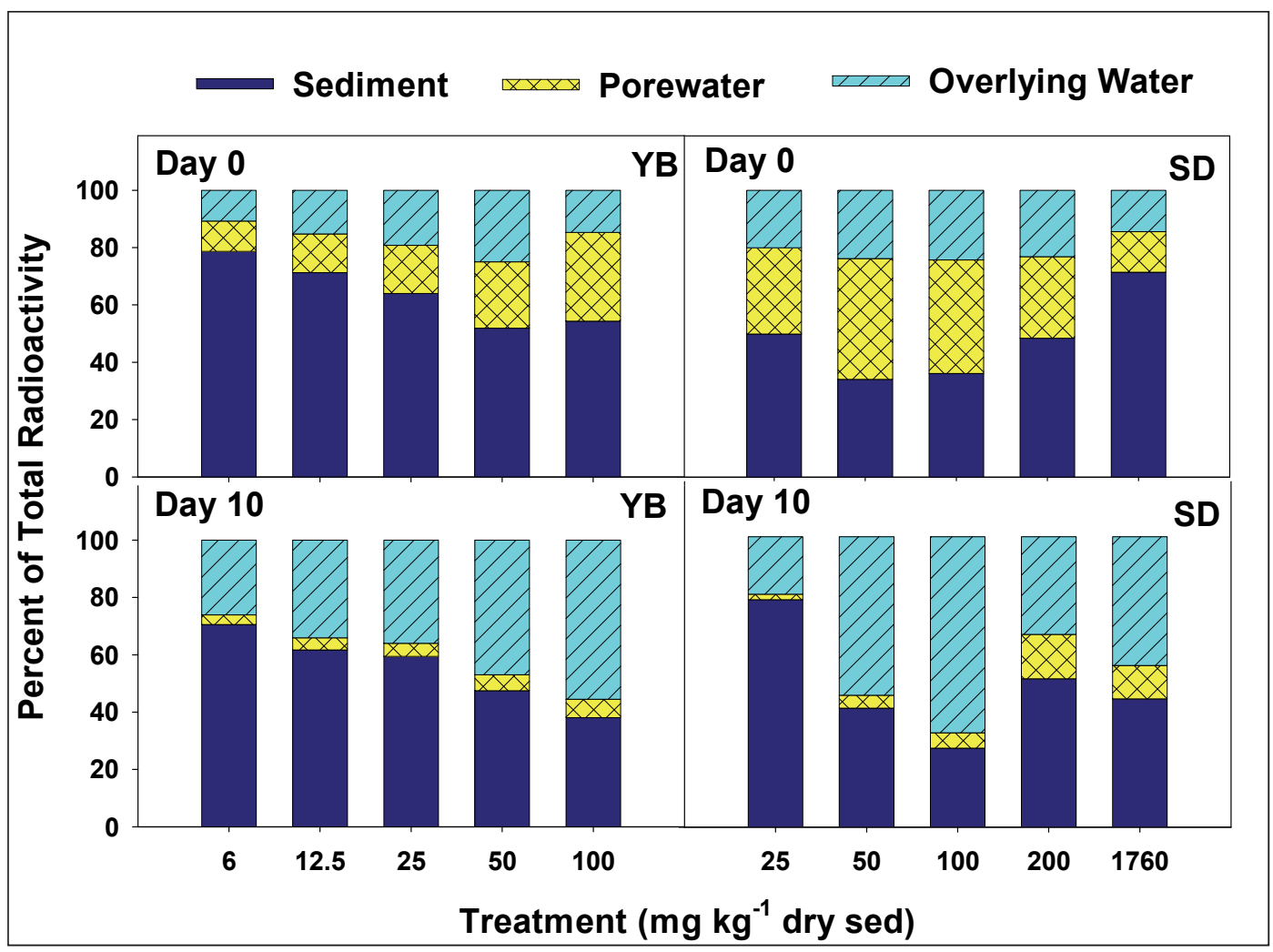

Figure 5.4. Percentage of total radioactivity from radiolabeled-TNT in the exposure chamber that was associated with various media at toxicity test initiation (Day 0) and termination (Day 10) for sandy sediment (Yaquina Bay, YB) or fine-grained sediment (San Diego Bay, SD) (from Rosen and Lotufo 2005). 
Because TNT is rapidly transformed in sediment and the concentration of sumTNT decreases during the toxicity exposures, establishing accurate concentration-response relationships for TNT and related compounds using data from sediment toxicity testing is challenging. In addition, partitioning of TNT and its transformation products to the overlying water renders the latter as a potentially significant source of contaminant uptake to test organisms, relative to the sediment compartment, confounding the extrapolation of sediment toxicity test results to realistic field scenarios.

The fate of 2,6-DNT in spiked marine sediments has been investigated using fine-grained and sandy marine sediments (Nipper et al. 2004). These studies showed that biotransformation of 2,6-DNT started soon after the initial contact of the chemical with the sediments and proceeded for several months. Transformation rates were greater in fine-grained than in sandy sediment. The transformation product 2-amino-6-nitrotolune (2-A-6-NT) was formed by reduction and the compound 2-nitrotoune (2-NT) was formed by loss of a nitro group. After prolonged incubation, these chemicals tended to be replaced by high molecular weight polymers, possibly derived from polymerization of 2,6-DNT or its transformation products. Complete disappearance of the parent compound and known transformation products from fine-grained and sandy sediments occurred during a 56-d incubation at $20^{\circ} \mathrm{C}$. The possibility of mineralization of at least part of the 2,6-DNT was speculated. Although the majority of 2,6-DNT in spiked sediments is biotransformed, abiotic transformation took place in sterilized sediments.

Analytically determined concentrations of 2,6-DNT and 2-A-6-NT, as well as picric acid, in marine sediments were much lower when samples were dried at room temperature and extracted with acetonitrile (Nipper et al. 2004), according to standard procedures for soils and sediments than when sediments were not dried prior to extraction. A portion of the parent compounds could be transformed during the drying procedure, and the authors suggest that sediments contaminated with explosives should not be air-dried prior to extraction for chemical measurements.

\section{Toxicity of nitroaromatic MC amended to sediment}

Due to the rapid transformation of MC spiked to sediment described above, target (or nominal) concentrations were higher, sometimes by orders of magnitude, than the actual, measured concentrations. Therefore, analytically determined concentrations measured at exposure initiation more accurately represent the effects concentrations than target concen- 
trations, especially when toxic response occurs shortly after exposure initiation. Rapid manifestation of toxicity is expected for TNT and its major transformation products as these chemicals reach steady state in small organisms very quickly (Section 6). Lethal effects of TNT were observed during the initial few days of exposure in freshwater sediment toxicity tests (Conder et al. 2004a; Lotufo and Farrar 2005). Therefore, the use of short exposure duration for the evaluation of the effects of sediment-associated explosives is desirable.

Results of toxicity investigations using sediments spiked with TNT and related compounds as well as other explosive compounds are summarized in Table 5.2. Due to the paucity of data for the marine environment, freshwater toxicity data was also included. For each study, no observed effect concentrations (NOEC) and lowest observed effect concentrations (LOEC) are provided as initial concentrations expressed as SumTNT concentrations. The earliest study of the toxicity of TNT in sediment (Green et al. 1999) employed ${ }^{14} \mathrm{C}$-labeled TNT and used radioactivity measurements as a surrogate for the sediment concentrations. Most of the radioactivity detected in the sediments in that study likely corresponded to non-solventextractable transformation products of TNT. This was later confirmed in similar spiked-sediment studies performed using sediments from the same site (Lotufo, unpublished study). Therefore, the effects concentrations reported in that study are likely substantially higher than the concentration of SumTNT, explaining the apparent high relative tolerance of the amphipod Leptocheirus plumulosus and polychaete Neanthes arenaceodentata to sediments spiked with this chemical. In the other studies in Table 5.2, sediment concentrations of SumTNT at initiation of the toxicity exposure were chemically measured, therefore providing more accurate determination of the exposure concentration suitable for expressing lethal toxicity.

Table 5.2. Lethal toxicity data for nitroaromatic MC determined for aquatic invertebrates using spiked sediment exposures.

\begin{tabular}{|l|l|l|l|l|l|l|l|}
\hline $\begin{array}{l}\text { Spiked } \\
\text { MC }\end{array}$ & Species & Habitat & $\begin{array}{l}\text { TOC } \\
(\%)\end{array}$ & $\begin{array}{l}\text { Duration } \\
(\mathbf{d})\end{array}$ & $\begin{array}{l}\text { NOEC } \\
\text { mg kg-1 }^{-1}\end{array}$ & $\begin{array}{l}\text { LOEC } \\
\text { mg kg-1 }^{-1}\end{array}$ & Reference \\
\hline TNT & $\begin{array}{l}\text { Amphipod } \\
\text { Leptocheirus } \\
\text { plumulosus }\end{array}$ & \begin{tabular}{l} 
Marine \\
\cline { 2 - 7 } \\
\cline { 2 - 8 } \\
Neanthes \\
arenaceodentata
\end{tabular} & 2.70 & 28 & 116 & 228 & $\begin{array}{l}\text { Green et al. } \\
1999\end{array}$ \\
\hline
\end{tabular}




\begin{tabular}{|c|c|c|c|c|c|c|c|}
\hline $\begin{array}{l}\text { Spiked } \\
\text { MC }\end{array}$ & Species & Habitat & $\begin{array}{l}\text { TOC } \\
(\%)\end{array}$ & $\begin{array}{l}\text { Duration } \\
\text { (d) }\end{array}$ & $\begin{array}{l}\text { NOEC } \\
\mathrm{mg} \mathrm{kg}^{-1}\end{array}$ & $\begin{array}{l}\text { LOEC } \\
\mathrm{mg} \mathrm{kg}^{-1}\end{array}$ & Reference \\
\hline & $\begin{array}{l}\text { Amphipod } \\
\text { Hyalella azteca }\end{array}$ & Freshwater & 0.65 & 10 & $\begin{array}{l}<0.1^{a} \\
(25)\end{array}$ & $0.1(50)$ & $\begin{array}{l}\text { Steevens et al. } \\
2002\end{array}$ \\
\hline & Midge (larva) & Freshwater & 0.65 & 10 & $4(100)$ & $67(200)$ & $\begin{array}{l}\text { Steevens et al } \\
2002\end{array}$ \\
\hline & Chironomus dilutes & Freshwater & 0.65 & 10 & ND & $37(43)$ & $\begin{array}{l}\text { Lotufo and } \\
\text { Farrar } 2005\end{array}$ \\
\hline & \begin{tabular}{|l} 
Oligochaete \\
Tubifex tubifex
\end{tabular} & Freshwater & 0.96 & 28 & $\begin{array}{l}25 \\
(100)\end{array}$ & $69(320)$ & $\begin{array}{l}\text { Conder et al. } \\
2004 a\end{array}$ \\
\hline & Amphipod & Marine & 0.09 & 10 & $24(50)$ & $44(100)$ & $\begin{array}{l}\text { Rosen and } \\
\text { Lotufo } 2005\end{array}$ \\
\hline & Eohaustorius estuarius & Marine & 0.36 & 10 & $\begin{array}{l}20 \\
(100)\end{array}$ & $38(200)$ & $\begin{array}{l}\text { Rosen and } \\
\text { Lotufo } 2005\end{array}$ \\
\hline \multirow{5}{*}{ 2,4-DANT } & $\begin{array}{l}\text { Leptocheirus } \\
\text { plumulosus }\end{array}$ & Marine & 2.70 & 28 & $\begin{array}{l}<0.1^{a} \\
(57)\end{array}$ & $3.0(113)$ & $\begin{array}{l}\text { Lotufo et al. } \\
2001\end{array}$ \\
\hline & \begin{tabular}{|l|} 
Neanthes \\
arenaceodentata
\end{tabular} & Marine & 2.70 & 28 & $\begin{array}{l}187 \\
(444)\end{array}$ & $\begin{array}{l}574 \\
(1,185)\end{array}$ & $\begin{array}{l}\text { Lotufo et al. } \\
2001\end{array}$ \\
\hline & Hyalella azteca & Freshwater & 0.65 & 10 & $\begin{array}{l}<0.1^{a} \\
(25)\end{array}$ & $0.3(50)$ & $\begin{array}{l}\text { Steevens et al. } \\
2002\end{array}$ \\
\hline & \multirow{2}{*}{ Chironomus dilutes } & Freshwater & 0.65 & 10 & $\begin{array}{l}110 \\
(400)\end{array}$ & ND & $\begin{array}{l}\text { Steevens et al } \\
2002\end{array}$ \\
\hline & & Freshwater & 0.65 & 10 & $\begin{array}{l}33 \\
(120)\end{array}$ & $59(243)$ & $\begin{array}{l}\text { Lotufo and } \\
\text { Farrar } 2005\end{array}$ \\
\hline $\begin{array}{l}\text { 2-Am- } \\
\text { DNT }\end{array}$ & Chironomus dilutes & Freshwater & 0.65 & 10 & $23(51)$ & $59(72)$ & $\begin{array}{l}\text { Lotufo and } \\
\text { Farrar } 2005\end{array}$ \\
\hline \multirow{5}{*}{$\begin{array}{l}1,3,5- \\
\text { TNB }\end{array}$} & $\begin{array}{l}\text { Leptocheirus } \\
\text { plumulosus }\end{array}$ & Marine & 2.70 & 28 & 7 (119) & $13(237)$ & $\begin{array}{l}\text { Lotufo et al. } \\
2001\end{array}$ \\
\hline & \begin{tabular}{|l|} 
Neanthes \\
arenaceodentata
\end{tabular} & Marine & 2.70 & 28 & $\begin{array}{l}107 \\
(468)\end{array}$ & ND & $\begin{array}{l}\text { Lotufo et al. } \\
2001\end{array}$ \\
\hline & Hyalella azteca & Freshwater & 0.65 & 10 & $\begin{array}{l}<0.1^{a} \\
(50)\end{array}$ & $1(100)$ & $\begin{array}{l}\text { Steevens et al } \\
2002\end{array}$ \\
\hline & \multirow{2}{*}{ Chironomus dilutes } & Freshwater & 0.65 & 10 & $\begin{array}{l}7.6 \\
(400)\end{array}$ & ND & $\begin{array}{l}\text { Steevens et al } \\
2002\end{array}$ \\
\hline & & Freshwater & 0.65 & 10 & $\begin{array}{l}59 \\
(140)\end{array}$ & $\begin{array}{l}131 \\
(289)\end{array}$ & $\begin{array}{l}\text { Lotufo and } \\
\text { Farrar } 2005\end{array}$ \\
\hline \multirow{2}{*}{ 2,6-DNT } & \multirow{2}{*}{$\begin{array}{l}\text { Amphipod } \\
\text { Ampelisca abdita }\end{array}$} & Marine & 0.10 & 10 & 5 & ND & $\begin{array}{l}\text { Nipper et al. } \\
2004\end{array}$ \\
\hline & & Marine & 1.10 & 10 & 0.5 & ND & $\begin{array}{l}\text { Nipper et al. } \\
2004\end{array}$ \\
\hline
\end{tabular}

TOC - total organic carbon, NOEC - No observed effect concentration, LOEC - lowest observed effect concentration, ND not determined due to insufficient mortality, NOEC and LOEC reported as sum concentrations of parent and transformation products measured at experiment initiation (when available, target concentrations are shown in parenthesis), a Numerical value represents detection limit . 
However, concentrations measured using solvent extraction of air-dried sediments (Lotufo et al. 2001; Steevens et al. 2002) likely underestimated the actual concentration of extractable compounds in the sediments and consequently resulted in the determination of lower than actual effects concentrations.

Except for H. azteca, lethality was observed remarkably similar SumTNT concentrations for a variety of experimental species and sediment organic carbon content. However, comparisons of the toxicity of TNT-spiked sediments using different sediments and species should take into account the variable relative concentration of parent and transformation products in the different exposure sediments, since there are potential toxicity differences among compounds and non-additive mixture interactions.

Complete mortality of juvenile sheepshead minnows (Cyprinodon variegatus) was observed after only $24 \mathrm{~h}$ of exposure to moderately organicrich sediment $(\mathrm{TOC}=1.1 \%)$ that had an initial Sum-TNT concentration of $348 \mathrm{mg} \mathrm{kg}^{-1}$, while no mortality was observed at an initial sediment concentration of $6.9 \mathrm{mg} \mathrm{kg}^{-1}$ (Lotufo et al. 2010b). Therefore, an $\mathrm{LC}_{50}$ would likely occur somewhere between these two concentrations. The study concluded that toxicity was unlikely to be caused by dermal uptake from sediment; rather, it was due to exposure to the overlying water which was not renewed during the experimental period and therefore contained lethal concentrations of TNT and its transformation products. No significant mortality of freckled blennies (Hypsoblennius ionthas) occurred in exposure to sediment Sum-TNT concentrations as high as $198 \mathrm{mg} \mathrm{kg}^{-1}$ (the highest concentration studied) for up to 7 days (Lotufo et al. 2010b).

Lotufo et al. (2001) suggested that with respect to lethality, L. plumulosus was more sensitive to $1,3,5$-TNB and 2,4-DANT than to TNT, whereas $N$. arenaceodentata was more sensitive to TNT than to $1,3,5$-TNB or 2,4DANT when comparing $\mathrm{LC}_{50}$ from this study and the study by Green et al. (2001), which also utilized the sediment for spiking. The TNT LC ${ }_{50}(203 \mathrm{mg}$ $\mathrm{kg}^{-1}$ for L. plumulosus and $320 \mathrm{mg} \mathrm{kg}^{-1}$ for $N$. arenaceodentata) were calculated using the sum concentration of TNT and all its transformation products in the sediment (Green et al. 1999). The 1,3,5-TNB and 2,4-DANT 28-d $\mathrm{LC}_{50}$ for L. plumulosus calculated on the basis of the measured amount of compound added to the sediment were $90.4 \mathrm{mg} \mathrm{kg}^{-1}$ for 2,4-DANT and $95.4 \mathrm{mg} \mathrm{kg}^{-1}$ for $1,3,5$-TNB; while a substantially higher $28-\mathrm{d} \mathrm{LC}_{50}$ value, 
$450.9 \mathrm{mg} \mathrm{kg}^{-1}$, was calculated for 2,4-DANT using $N$. arenaceodentata (Lotufo et al. 2001).

The toxicity of TNT, 2-Am-DNT, 2,4-DANT spiked to freshwater sediment was compared for the midge Chironomus dilutus (Lotufo and Farrar 2005) using the same sediment matrix and exposure conditions. Lethal toxicity was similar for all compounds, with differences in LOEC values never exceeding a factor of 4 (Table 5.2). Overall, reduced transformation products of TNT appear to be less lethal than TNT to marine and freshwater invertebrates (Table 5.2). Toxic interaction among TNT and its major transformation products in spiked sediment exposures was additive in the single study addressing the mixture toxicity of nitroaromatic compounds in sediment, suggesting the toxicity of a nitroaromatic mixture would be reasonably predicted using single-compound toxicity data (Lotufo and Farrar 2005).

The broad range of toxicity of TNT and related compounds in spikedsediment exposures likely resulted from differences in species sensitivity, compound bioavailability and the relative concentration of parent and transformation compounds in the exposure sediments. Inaccuracies in quantifying exposure concentrations likely contributed to the observed differences among studies.

The toxicity of fine-grained and sandy sediments spiked with 2,6-DNT was examined using the amphipod Ampelisca abdita in 10-d exposures (Nipper et al. 2004). No significant lethal effects were observed in any treatment (Table 5.2). Since the highest 2,6-DNT measured concentration in sediments used in toxicity evaluations was relatively low $\left(5 \mathrm{mg} \mathrm{kg}^{-1}\right)$, the effects of 2,6-DNT at concentrations representative of the lethal range for other nitroaromatic compounds are unknown. Additional reports of the toxicity of sediments spiked with 2,6-DNT transformation products have not been found in the available literature.

\section{Fate and toxicity of nitrophenolic MC in sediment}

The fate of picric acid in marine sediments was investigated by Nipper et al. (2004) using the same sediments and experimental conditions described above for 2,6-DNT. Transformation rates were highest in fine-grained sediment incubated at $20^{\circ} \mathrm{C}$. Major transformation products included 4-dinitrophenol, aminodinitrophenols (including picramic acid), 3,4-diaminophenol, amino nitrophenol, and nitrodiaminophenol. High 
performance liquid chromatography analysis of the sediment extracts revealed unknown compounds speculated to correspond to aminonitrotoluene isomers, diaminotoluenes, dinitrotoluenes, and nitrotoluenes. Complete degradation of the parent compound and known transformation products occurred during the 56 - $d$ storage period, but unidentified transformation products remained in the sandy and fine-grained sediments. Similar to the fate of 2,6-DNT, picric acid disappeared from sterilized spiked marine sediments, suggesting a certain degree of abiotic transformation.

The toxicity of both sediment types spiked with picric acid was examined using $A$. abdita in 10-d exposures (Nipper et al. 2002). Significant amphipod mortality was observed in the sandy sediment at picric acid concentrations relatively similar to lethal concentrations reported for TNT and its major breakdown products (Table 5.3). In the fine-grained sample, mortality was highest at an intermediate concentration in which picric acid was below the detection limit, with greater survival in the two higher and lower concentrations, thus generating a U-shaped concentration-response relationship. The toxicity in treatments with picric acid below detection limit was attributed to unknown transformation products. Inhibition of microbial transformation at the highest concentrations tested in that study could have contributed to the lower mortality at higher concentrations compared to mortality at the intermediate concentrations.

Table 5.3. Toxicity of nitrophenolic MC was determined for the marine amphipod Ampelisca abdita using spiked sediment exposures (Nipper et al. 2002).

\begin{tabular}{|l|l|l|l|l|l|}
\hline MC & Sediment & $\begin{array}{l}\text { TOC } \\
(\%)\end{array}$ & $\begin{array}{l}\text { Duration } \\
(\mathrm{d})\end{array}$ & $\begin{array}{l}\text { NOEC } \\
\mathrm{mg} \mathrm{kg}^{-1}\end{array}$ & $\begin{array}{l}\text { LOEC } \\
\mathrm{mg} \mathrm{kg}^{-1}\end{array}$ \\
\hline Picric acid & Sand & 0.10 & 10 & 73 & 162 \\
\hline Picric acid & Fine-grained & 1.10 & 10 & BDL & BDL \\
\hline Tetryl & Sand & 0.10 & 10 & 0.5 & 4 \\
\hline Tetryl & Fine-grained & 1.10 & 10 & 0.1 & ND \\
\hline
\end{tabular}

NOEC - No observed effect concentration, LOEC - lowest observed effect concentration, NOEC and LOEC reported as sum concentrations of parent and transformation products measured at experiment initiation (target concentration not reported), BDL - Significant mortality occurred but concentrations of picric acid and its transformation products were below the detection limit, ND - not determined due to insufficient mortality in all treatments.

Information on the fate of tetryl in sediments is limited to a study by Nipper et al. (2002). Unknown breakdown products were formed in sandy and fine-grained marine sediments spiked with tetryl. Picric acid, a primary hydrolysis product of tetryl in seawater, was detected in the sandy sediment 
only. Tetryl-spiked sandy sediments produced significant amphipod mortality at relatively low concentrations in a 10-d exposure using $A$. abdita (Table 5.3). Significant lethal effects were not observed in the tetryl-spiked fine-grained sediment (Table 5.3), in which the highest concentration (0.1 $\mathrm{mg} \mathrm{kg}{ }^{-1}$ ) was much lower than lethal levels in the sandy sediment. Since tetryl has not been produced in the United States since the early 1970s and the quantities in individual munitions were small, recent research efforts to understand its fate and effects in sediments were minimal. However, given its elevated toxicity to a variety of species (Section 4), the need for additional studies of its fate and biological effects in different types of sediment should be considered.

\section{Toxicity and fate of nitramine MC in sediment}

The cyclic nitramine explosives RDX and HMX have lower water solubility and significantly less binding affinity with soil organic matter than TNT (Table 3.1). Therefore, major differences in the fate of those compounds in aquatic systems are expected. Studies using ${ }^{14} \mathrm{C}$-labeled compounds suggested, but did not conclusively demonstrate by chemical analysis of transformation products, that RDX and HMX spiked into fine-grained, organically rich sediments were likely degraded during the course of toxicity experiment (Lotufo et al. 2001). A substantial portion of the radioactivity originally associated with RDX- or HMX-spiked sediments partitioned to the overlying water in exposure chambers during the course of toxicity tests using fine-grained sediment and was present in that compartment at exposure termination (Figure 5.5). That radioactivity was likely associated with transformation products more soluble in water than the parent compounds. Radiolabel mass balance revealed substantial losses of radioactivity from exposure chambers in static exposures to RDX, which may have resulted from volatilization of RDX transformation products or loss via mineralization.

Different from the fate of RDX in organically rich sediment, transformation was minimal in sandy sediment, and a much smaller fraction of the spiked radioactivity was associated with the overlying water at termination of a 10-d toxicity exposure (Figure 5.6) (Rosen and Lotufo 2005). In the latter study, RDX was added to sediments in excessive amounts, up to $0.24 \%$ of the sediment dry mass, to attain concentrations expected to promote toxicity to an amphipod. In that study, most of the spiked RDX remained in the sediment/porewater compartment of the exposure chamber and likely corresponded to undissolved crystals rather than to compound bound to 
sediment particles. RDX has demonstrated low sorption affinity to soil particles, with a concentration of $80 \mathrm{mg} \mathrm{kg}^{-1}$ or greater creating a saturated porewater (Savard et al. 2010).

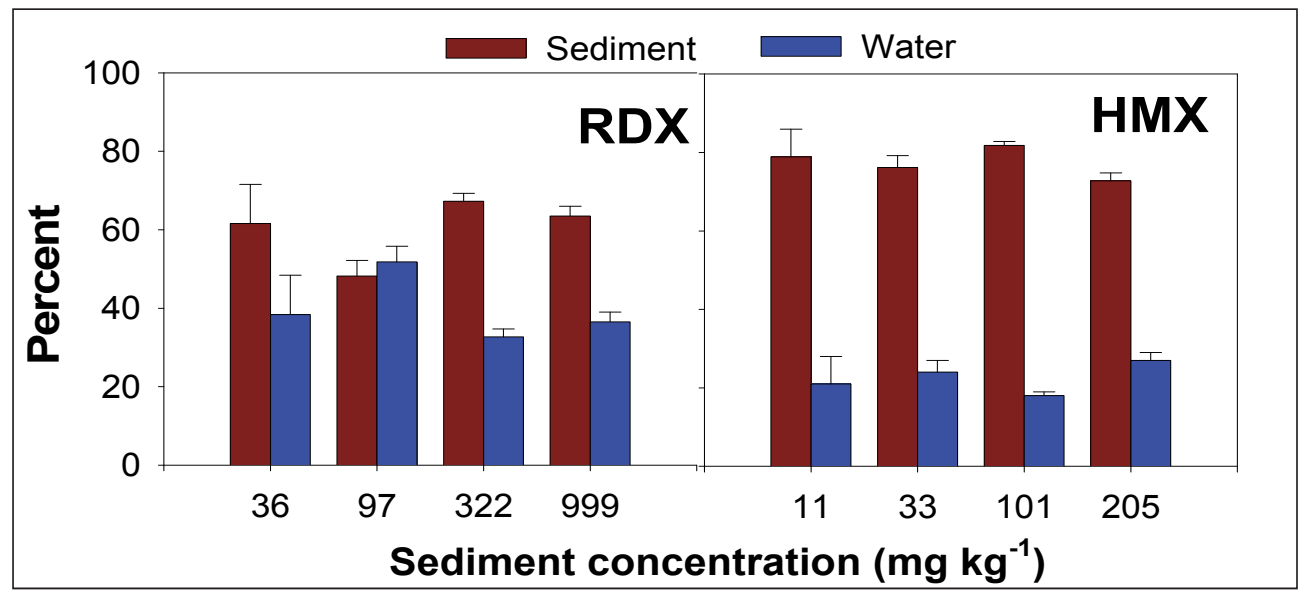

Figure 5.5. Percentage of the total radioactivity from RDX or HMX in the exposure chamber that was associated with sediment and overlying water at termination of a toxicity test (from Lotufo et al. 2001). Error bars represent \pm 1 standard deviation.

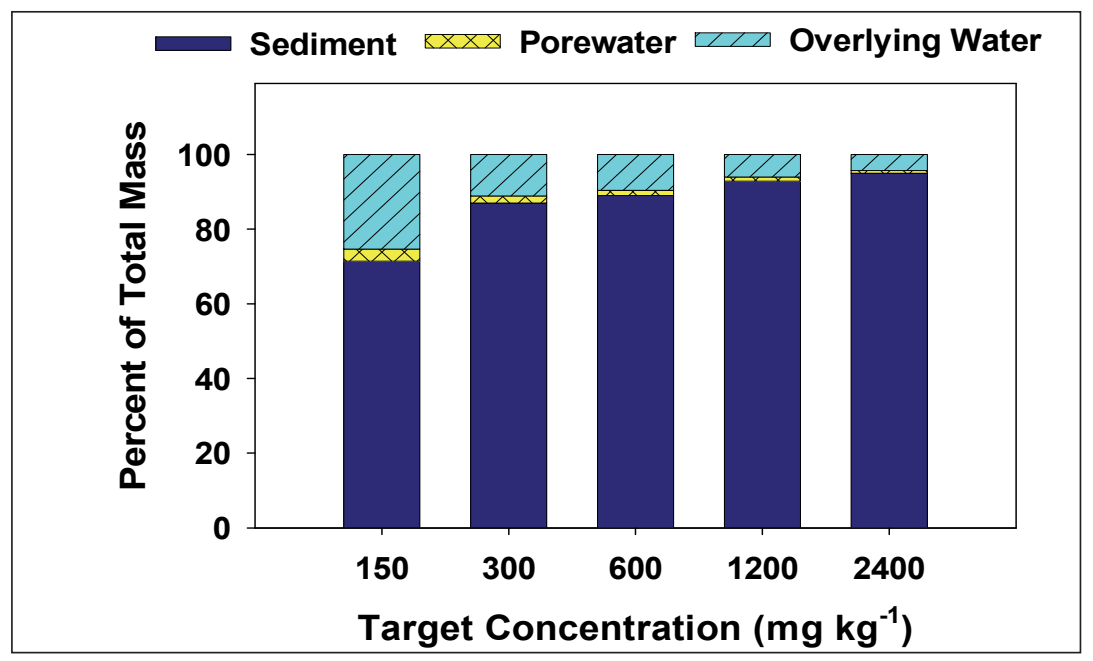

Figure 5.6. Percentage of the total mass of RDX in the exposure chamber that was associated with various media at toxicity test termination for a sandy sediment (from Rosen and Lotufo 2005).

The toxicities of RDX or HMX were investigated using spiked fine-grained marine sediments, with L. plumulosus and $N$. arenaceodentata 10-d exposures (Lotufo et al. 2001). The toxicity of RDX was also investigated using sandy sediment (Rosen and Lotufo 2005). No significant mortality was observed in any exposure, even when the measured concentration approached or exceeded $1 \mathrm{~g} \mathrm{~kg}^{-1}$ (Table 5.4). Reports of the toxicity of RDX or HMX transformation products in sediment exposures have not been found in the available literature. 
Table 5.4. Toxicity of RDX and HMX determined for aquatic invertebrates using spiked sediment exposures.

\begin{tabular}{|c|c|c|c|c|c|c|c|}
\hline $\begin{array}{l}\text { Spiked } \\
\text { chemical }\end{array}$ & Species & Habitat & \begin{tabular}{|l|} 
TOC \\
$(\%)$
\end{tabular} & $\begin{array}{l}\text { Duration } \\
\text { (d) }\end{array}$ & $\begin{array}{l}\text { NOEC } \\
\mathrm{mg} \mathrm{kg}^{-1}\end{array}$ & $\begin{array}{l}\text { LOEC } \\
\mathrm{mg} \mathrm{kg}^{-1}\end{array}$ & Reference \\
\hline \multirow{5}{*}{ RDX } & \begin{tabular}{|l|} 
Amphipod \\
Leptocheirus \\
plumulosus
\end{tabular} & Marine & 2.70 & 10 & 891 & ND & Lotufo et al. 2001 \\
\hline & \begin{tabular}{|l|} 
Polychaete \\
Neanthes \\
arenaceodentata
\end{tabular} & Marine & 2.70 & 10 & 891 & ND & Lotufo et al. 2001 \\
\hline & \begin{tabular}{|l|} 
Amphipod \\
Eohaustorius estuarius
\end{tabular} & Marine & 0.09 & 10 & 2229 & ND & $\begin{array}{l}\text { Rosen and Lotufo } \\
2005\end{array}$ \\
\hline & $\begin{array}{l}\text { Amphipod } \\
\text { Hyalella azteca }\end{array}$ & Freshwater & 0.65 & 10 & 102 & ND & $\begin{array}{l}\text { Steevens et al } \\
2002\end{array}$ \\
\hline & \begin{tabular}{|l} 
Midge (larva) \\
Chironomus dilutus
\end{tabular} & Freshwater & 0.65 & 10 & 711 & ND & $\begin{array}{l}\text { Steevens et al } \\
2002\end{array}$ \\
\hline \multirow{4}{*}{ HMX } & \begin{tabular}{|l}
$\begin{array}{l}\text { Leptocheirus } \\
\text { plumulosus }\end{array}$ \\
\end{tabular} & Marine & 2.70 & 10 & 115 & ND & Lotufo et al. 2001 \\
\hline & $\begin{array}{l}\text { Neanthes } \\
\text { arenaceodentata }\end{array}$ & Marine & 2.70 & 10 & 353 & ND & Lotufo et al. 2001 \\
\hline & Hyalella azteca & Freshwater & 0.65 & 10 & 126 & ND & $\begin{array}{l}\text { Steevens et al } \\
2002\end{array}$ \\
\hline & Chironomus dilutus & Freshwater & 0.65 & 10 & 146 & ND & $\begin{array}{l}\text { Steevens et al } \\
2002\end{array}$ \\
\hline
\end{tabular}

TOC - Total organic carbon, NOEC - No observed effect concentration, LOEC - lowest observed effect concentration, NOEC and LOEC reported as concentrations measured at experiment initiation, ND - not determined due to insufficient mortality in all treatments. See Table 4.2 for species habitat information.

\section{Sublethal toxicity and stimulatory effects of MC in sediment exposures}

Few records of biological effects other than lethality are available for sediment exposures to explosives. In studies where effects other than mortality were investigated, non-lethal effects (i.e., significant decreases in growth (Steevens et al. 2002) or reproduction (Conder et al. 2004a, Green et al. 1999) were reported only in treatments where survival was also significantly decreased and therefore were not truly sublethal manifestations of sediment exposure to explosives. However, a sublethal decrease in offspring production was observed in L. plumulosus and a sublethal decrease in biomass was exhibited by juvenile $N$. arenoceodentata at concentrations a few fold lower than lethal concentrations (Lotufo et al. 2001). Lethal effects of RDX to L. plumulosus and N. arenoceodentata were not observed (Lotufo et al. 2001) (Table 5.4). To further elucidate lethal and sublethal effects on growth and reproduction, additional studies using a variety of benthic invertebrates are warranted. 
Exposure to explosive-spiked sediments has promoted stimulatory effects on growth of a few species of freshwater invertebrates. Significantly increased biomass was observed for $C$. dilutus exposed to RDX, HMX (Steevens et al. 2002), TNT, and 1,3,5-TNB (Lotufo and Farrar 2005) in sediment treatments that did not cause significant lethality. Similar effects were observed for $H$. azteca exposed to 1,3,5-TNB or RDX (Steevens et al. 2002) and for N. arenaceodentata exposed to TNT (Green et al. 1999). The mechanisms responsible for hormetic-like concentration-response relationships reported in these studies are unknown but were speculated to be related to explosives-induced changes in the nutritional status of the sediment and biochemical and physiological alterations in invertebrates (Green et al. 1999).

\section{Toxicity of porewater extracted from sediments spiked with explosives}

The toxicity of porewaters from fine-grained and sandy marine sediments spiked individually with 2,6-DNT, tetryl, or picric acid, and allowed to equilibrate at $4{ }^{\circ} \mathrm{C}$ for $5-10$ days, varied with both the compound and the nature of the spiked sediment (Nipper et al. 2002). Toxicity was assessed using sea urchin (Arbacia punctulata) embryological development, macroalga (Ulva fasciata) zoospore germination and germling growth, and polychaete (Dinophilus gyrociliatus) survival and reproduction tests. Tetryl and picric acid were the most and least toxic of the three chemicals, respectively, in porewaters from both kinds of sediments (Nipper et al. 2002). Samples spiked with 2,6-DNT contained a transformation product identified as 2-A-6-NT. Unidentified peaks, possibly transformation products, were also seen in some of the picric acid- and tetryl-spiked samples. Porewater from the fine-grained sediment spiked with 2,6-DNT and tetryl was up to three orders of magnitude more toxic than porewater from the sandy sediment (Table 5.5), possibly due to the presence of relatively toxic non-identified transformation products.

Further assessments were performed of the toxicity of transformation products of 2,6-DNT and picric acid in porewaters from spiked sandy and fine-grained marine sediments over a 6-month sediment incubation period at 10 and $20^{\circ} \mathrm{C}$. During these assessments, incremental transformation was reported (Nipper et al. 2005). Toxicity of porewater from sediments spiked with 2,6-DNT decreased for the macroalga, $U$. fasciata zoospores, with sediment incubation time as transformation progressed, but increased for the copepod, Schizopera knabeni, nauplii. The porewater of fine-grained sediment spiked with either 2,6-DNT or picric acid had lower toxicity than 
Table 5.5. Toxicity of marine porewater extracted from sandy or fine-grained sediments spiked with 2,6-DNT, tetryl or picric acid (from Nipper et al. 2002).

\begin{tabular}{|c|c|c|c|c|c|}
\hline \multirow{3}{*}{$\begin{array}{l}\text { Sediment } \\
\text { type }\end{array}$} & \multirow[b]{3}{*}{ MC } & Urchin & \multicolumn{2}{|c|}{ Polychaete } & \multirow{2}{*}{\begin{tabular}{|l|} 
macroalga zoospore \\
Germling length \\
EC $_{50}$
\end{tabular}} \\
\hline & & $\begin{array}{l}\text { Embryo development } \\
\text { EC }_{50}\end{array}$ & $L_{50}$ & $\begin{array}{l}\text { Reproduction } \\
\text { EC }_{50}\end{array}$ & \\
\hline & & \multicolumn{4}{|c|}{$\mathrm{mg} \mathrm{L}^{-1}$} \\
\hline Sandy & 2,6-DNT & 36.9 & 21.1 & 8.16 & 3.28 \\
\hline Fine grained & 2,6-DNT & 0.043 & 0.046 & 0.023 & $\begin{array}{l}\text { No effects at } \\
0.087 \text { a }\end{array}$ \\
\hline Sand & Tetryl & 0.27 & 0.055 & 0.66 & 0.48 \\
\hline Fine grained & Tetryl & 0.00056 & 0.0019 & 0.0019 & $\begin{array}{l}\text { No effects at } \\
0.003^{a}\end{array}$ \\
\hline Sand & $\begin{array}{l}\text { Picric } \\
\text { acid }\end{array}$ & 592.8 & 127.5 & 83.4 & 83.1 \\
\hline Fine grained & $\begin{array}{l}\text { Picric } \\
\text { acid }\end{array}$ & 190.2 & 170.4 & 64.8 & 11.22 \\
\hline
\end{tabular}

EC50 - median effective concentration, $\mathrm{LC}_{50}$ - median lethal concentration, a Highest concentration tested.

its sandy counterpart after 6 months, suggesting faster microbial transformation in fine-grained sediments, resulting in further transformation of picramic acid and 2,4-DNP into less toxic products (Nipper et al. 2005). Overall, transformation products of picric acid and 2,6-DNT were more toxic than their parent compounds, but that transformation, primarily of biotic nature, proceeds towards the formation of less toxic products given sufficient time.

\section{Preliminary sediment quality benchmarks}

Sediment screening criteria for many MC are not available in sources typically used in regulatory-driven ecological risk assessments for contaminated sediment sites. Preliminary sediment quality benchmarks (SQBs) for MC were developed for screening potential risks to marine benthic invertebrates at an MC-contaminated sediment site in Puget Sound, WA by Pascoe et al. (2010). Those SQBs were developed using freshwater and limited marine water-only toxicity values and equilibrium partitioning theory to relate water concentrations of the compounds to sediment concentrations. Pascoe et al. (2010) presented SQBs for $25 \mathrm{MC}$ as organic carbon-normalized values and as ranges of dry weight values for various levels of organic carbon content of sediments. Comparison of the preliminary SQBs with method detection limits and sample detection 
limits achieved at the contaminated sediment site demonstrates their utility in risk screening of benthic invertebrates.

Sediment toxicity values, expressed as NOECs, for nine MC based on sediment spiking experiments compiled in Lotufo et al. (2009a), and the dry weight SQB values derived Pascoe et al. (2010) are compared in Table 5.6. The spiked sediment NOECs for all MC except 2,6-DNT are substantially higher than the SQB values. The differences between equilibrium partitioning-based SQBs and spiked sediment NOECs reported in Lotufo et al. (2009a) are related to the uncertainties in quantifying exposures in spiked sediment due to chemical and biological transformation, as discussed in Lotufo et al. (2009a) and in the uncertainty associated with assuming equilibrium between sediment and porewater (Pascoe et al. 2010).

Table 5.6. Marine sediment quality benchmarks (SQB) and no observed effect concentration (NOEC) from sediment toxicity tests for nine MC.

\begin{tabular}{|l|l|l|l|}
\hline \multirow{2}{*}{ MC } & \multicolumn{2}{|c|}{$\begin{array}{c}\text { SQBa } \\
\left(\mathrm{mg} \mathrm{kg}^{-1}\right)\end{array}$} & \multicolumn{1}{|c|}{$\begin{array}{l}\text { Spiked Sediment NOEC } \\
\left(\mathrm{mg} \mathrm{kg}^{-1}\right)\end{array}$} \\
\cline { 2 - 4 } & Low & High & $<0.1$ \\
\hline TNT & 0.0055 & 0.064 & 23 \\
\hline 2-Am-DNT & 0.0065 & 0.0075 & $<0.1$ \\
\hline $2,4-D A N T$ & 0.00045 & - & 0.500 \\
\hline $2,6-D N T$ & 2.1 & 2.7 & $<0.1$ \\
\hline 1,3,5-TNB & 0.0008 & 0.0043 & 73 \\
\hline Picric acid & 1.7 & -- & 0.1 \\
\hline Tetryl & 0.003 & 0.031 & 126 \\
\hline HMX & 0.0019 & 0.215 & 102 \\
\hline RDX & 0.006 & 0.039 & \\
\hline
\end{tabular}

a values derived Pascoe et al. (2010), b sediment toxicity data compiled in Lotufo et al. (2009a) 


\section{Toxicokinetics and Bioconcentration of Munitions Constituents}

This section summarizes and discusses the bioconcentration, bioaccumulation, biotransformation and toxicokinetic processes of nitroaromatic and cyclic nitramine $\mathrm{MC}$ in aquatic organisms. Due to the paucity of information on this subject from studies investigating marine organisms, information from freshwater studies are also included in this overview. Investigations of the bioconcentration and biotransformation of nitrophenolic MC and nitrate esters have not been found in the available literature.

\section{Overview}

Bioaccumulation is the process that causes an increased chemical concentration in an organism compared to that in its ambient environment, through all exposure routes, including dietary absorption and transport across body surfaces (Borga et al. 2012). Bioaccumulation includes uptake by respiration of chemical from the environment surrounding the organism and dietary exposures. Major routes of chemical elimination include loss by respiration, fecal egestion, urinary excretion, metabolic biotransformation, and growth dilution (Arnot 2009).

Bioconcentration is the process by which a chemical is absorbed by an organism from the water only through its respiratory and dermal surfaces. It is the net result of competing rates of uptake and elimination, including biotransformation (Arnot and Gobas 2006). MC compounds are weakly hydrophobic, and therefore, according to predictive models (e.g., Meylan et al. 1999; Arnot and Gobas 2003), their potential to bioconcentrate is low. Reports on the bioconcentration potential of $\mathrm{MC}$ in aquatic animals (reviewed in Lotufo et al. 2009b; Lotufo 2011) are scarce. In addition, studies examining the uptake from water, as well as elimination and biotransformation potential were conducted only for a few compounds using a small number of aquatic species (reviewed in Lotufo et al. 2009b).

Biomagnification can be regarded as a special case of bioaccumulation in which the chemical concentration in the organism exceeds that in its prey due to dietary absorption occurring faster than elimination (Gobas and 
Morrison 2000). The octanol-water partition coefficient, Kow, represents the hydrophobicity of a chemical and how it thermodynamically distributes between aqueous and organic phases (Arnot 2009). In aquatic food webs, poorly metabolized hydrophobic chemicals with $\log \mathrm{K}_{\mathrm{OW}}>5$ generally biomagnify, whereas chemicals with $\log \mathrm{K}_{\mathrm{OW}}<5$, even if poorly metabolized, do not (Gobas et al. 1999). Most explosive MC have log Kow less than 2 and all of the common explosive MC have a log Kowless than 4 (Table 3.1). Consequently, the chemicals are not expected to transfer efficiently through the dietary route and subsequently magnify. This expectation was confirmed in laboratory studies showing that the dietary uptake of $\mathrm{MC}$ in fish was minimal (reviewed in Lotufo et al. 2009b).

\section{Bioconcentration of MC}

A bioconcentration factor (BCF) is the ratio of a chemical concentration in an aquatic organism to that in the surrounding water, and is the most frequently used indicator of a compound's propensity to bioconcentrate in aquatic organisms (Meylan et al. 1999). Bioconcentration factors are determined primarily using steady state concentrations, or by using kinetically derived uptake and elimination rates (Lotufo and Lydy 2005). Bioconcentration factors reported for explosives and related compounds in invertebrate and fish species are provided in Table 6.1.

The BCFs for TNT ranged widely from 0.3 to $9.7 \mathrm{ml} \mathrm{g}^{-1}$ for a variety of marine and freshwater invertebrates and fish species (Conder et al. 2004b; Lotufo and Lydy 2005, Belden et al. 2005a; Ownby et al. 2005; Yoo et al. 2006; Rosen and Lotufo 2007b). Comparison of the bioconcentration of TNT and its major biotransformation products in the oligochaete Tubifex tubifex yielded lower BCFs for TNT and 2,4-DANT than for 2-Am-DNT and 4-Am-DNT (Conder et al. 2004b). A similar comparative study with sheepshead minnows (Cyprinodon variegatus) revealed similar BCFs for TNT and 2-Am-DNT, and a lower BCF value for 2,4-DANT (Lotufo and Lydy 2005).

The RDX BCFs were similar for channel catfish (Belden et al. 2005b), zebrafish (Muhki and Patino 2008), sheepshead minnows (Lotufo and Lydy 2005) and in the oligochaete Lumbriculus variegatus (Belden et al. 2005b). The RDX BCFs were lower for the mussel Mytilus galloprovincialis (Rosen and Lotufo 2007b), ranging from 0.8 to $2.1 \mathrm{ml} \mathrm{g}^{-1}$. The HMX bioconcentration in aquatic organisms was low, with a reported BCF of $0.51 \mathrm{ml} \mathrm{g}^{-1}$ for juvenile sheepshead minnows (Lotufo and Lydy 2005) and $0.31 \mathrm{ml} \mathrm{g}^{-1}$ for adult mussels (Rosen and Lotufo 2007b). 
Table 6.1. Empirically determined bioconcentration factor (BCF) values for fish and aquatic invertebrates.

\begin{tabular}{|c|c|c|c|c|c|}
\hline MC & Species & Habitat & Duration (d) & \begin{tabular}{|l} 
BCF \\
$\left(\mathrm{ml} \mathrm{g}^{-1}\right)$
\end{tabular} & Reference \\
\hline \multirow[t]{7}{*}{ TNT } & \multirow{2}{*}{$\begin{array}{l}\text { Sheepshead minnow } \\
\text { Cyprinodon variegatus }\end{array}$} & Marine & 0.25 & 9.71 & Lotufo and Lydy 2005 \\
\hline & & Marine & 1 & 3.3 & Lotufo 2011 \\
\hline & $\begin{array}{l}\text { Fathead minnow } \\
\text { Pimephales promelas }\end{array}$ & Freshwater & 0.25 & $2.3,5.0$ & Yoo et al. 2006 \\
\hline & $\begin{array}{l}\text { Channel catfish } \\
\text { Ictalurus punctuatus }\end{array}$ & Freshwater & 0.3 & 0.8 & Ownby et al. 2005 \\
\hline & $\begin{array}{l}\text { Mussel (adult) } \\
\text { Mytilus galloprovincialis }\end{array}$ & Marine & 0.17 & 0.31 & Rosen and Lotufo 2007b \\
\hline & $\begin{array}{l}\text { Oligochaete } \\
\text { Lumbriculus variegatus }\end{array}$ & Freshwater & 1 & 4 & Belden et al. $2005 a$ \\
\hline & $\begin{array}{l}\text { Oligochaete } \\
\text { Tubifex tubifex }\end{array}$ & Freshwater & 2.2 & 2.5 & Conder et al. 2004b \\
\hline \multirow[t]{2}{*}{ 2-Am-DNT } & Cyprinodon variegatus & Marine & 0.25 & 13.1 & Lotufo and Lydy 2005 \\
\hline & Tubifex tubifex & Freshwater & 2.2 & 10.2 & Conder et al. 2004b \\
\hline 4-Am-DNT & Tubifex tubifex & Freshwater & 2.2 & 12.4 & Conder et al. $2004 \mathrm{~b}$ \\
\hline \multirow[t]{2}{*}{ 2,4-DANT } & Cyprinodon variegatus & Marine & 0.25 & 0.51 & Lotufo and Lydy 2005 \\
\hline & Tubifex tubifex & Freshwater & 2.2 & 2.8 & Conder et al. $2004 \mathrm{~b}$ \\
\hline \multirow[t]{2}{*}{ 2,4-DNT } & $\begin{array}{l}\text { Common carp } \\
\text { Cyprinus carpio }\end{array}$ & Freshwater & 3 & 9.2 & Lang et al. 1997 \\
\hline & \begin{tabular}{|l} 
Goldfish \\
Carassius auratus
\end{tabular} & Freshwater & 20 & 4.5 & Wang et al. 1999 \\
\hline 2,6-DNT & Carassius auratus & Freshwater & 20 & 1.7 & Wang et al. 1999 \\
\hline \multirow[t]{4}{*}{ RDX } & \multirow{2}{*}{ Cyprinodon variegatus } & Marine & 0.25 & 1.7 & Lotufo and Lydy 2005 \\
\hline & & Marine & 1 & 0.7 & Lotufo 2011 \\
\hline & Ictalurus punctuatus & Freshwater & 0.7 & 2.0 & Belden et al. 2005b \\
\hline & $\begin{array}{l}\text { Zebra fish } \\
\text { Danio rerio }\end{array}$ & Freshwater & 28 & 1.0 & Mukhi and Patino 2008 \\
\hline RDX & Lumbriculus variegatus & Freshwater & 0.7 & 2.1 & Belden et al. 2005b \\
\hline RDX & Mytilus galloprovincialis & Marine & 0.17 & 0.71 & Rosen and Lotufo 2007b \\
\hline \multirow[t]{2}{*}{ HMX } & Cyprinodon variegatus & Marine & 0.25 & 0.51 & Lotufo and Lydy 2005 \\
\hline & Mytilus galloprovincialis & Marine & 0.17 & 0.31 & Rosen and Lotufo $2007 \mathrm{~b}$ \\
\hline
\end{tabular}

Various predictive models have been proposed to estimate BCFs for organic compounds based on their hydrophobicity as represented by their noctanol-water partition coefficient $\left(\mathrm{K}_{\mathrm{ow}}\right)$ (Meylan et al. 1999). Explosive compounds are weakly hydrophobic (low $\mathrm{K}_{\mathrm{ow}}$ values) and therefore BCFs predicted using these models should be low. The equation derived by 
Meylan et al. $\left(\log \mathrm{BCF}=0.86 \log \mathrm{K}_{\mathrm{ow}}\right.$ - 0.39) for non-ionic compounds in the $\log \mathrm{K}_{\mathrm{ow}}$ range of 1 to 7 was used to calculate predicted BCFs for explosives and related compounds (Table 6.2) for empirically derived BCF values reported in Table 6.1. A comparison of predicted and empirically derived $\mathrm{BCFs}$ revealed that most explosives and related compounds bioconcentrate in aquatic invertebrates and fish at concentrations lower than those predicted using their hydrophobicity (Table 6.2). Most measured BCFs were similar to model-predicted BCFs within a factor of 3. Overall, the bioconcentration of nitroaromatic and nitramine $\mathrm{MC}$ appear to be adequately predicted using a generic relationship derived for nonionic compounds suggesting that the bioconcentration of those compounds is a thermodynamically driven partitioning process between the water and the lipid phase of the exposed organisms.

Table 6.2. A comparison of predicted versus measured bioconcentration factor (BCF) values.

\begin{tabular}{|l|l|l|l|}
\hline MC & $\begin{array}{l}\text { log Kow } \\
\text { (from Table 2.2) }\end{array}$ & $\begin{array}{l}\text { Predicted BCF } \\
\left(\mathrm{ml} \mathrm{g}^{-1}\right)\end{array}$ & $\begin{array}{l}\text { Measured BCF } \\
\left(\mathrm{ml} \mathrm{g}^{-1}\right)\end{array}$ \\
\hline $2,4-D N T$ & 1.98 & 20.6 & 4.5 and 9.2 \\
\hline $2,6-D N T$ & 2.02 & 26.1 & 1.7 \\
\hline TNT & 1.60 & 9.7 & $0.31-9.71$ \\
\hline $2-$ Am-DNT & 1.94 & 19.3 & 10.2 and 13.1 \\
\hline $4-$ Am-DNT & 1.91 & 26.1 & 12.4 \\
\hline $2,4-D A N T$ & 0.79 & 2.0 & 0.51 and 2.8 \\
\hline RDX & 0.90 & 2.3 & 0.7 and 2.0 \\
\hline HMX & 0.17 & 0.6 & 0.31 to 0.51 \\
\hline
\end{tabular}

$\mathrm{BCF}$ predicted from log $\mathrm{K}_{\mathrm{ow}}$ values using a regression model (log BCF $\left.=0.86 \log \mathrm{K}_{\mathrm{ow}}-0.39\right)$ from Meylan et al. (1999) and the range of experimentally-determined values for fish and aquatic invertebrates are from Table 6.1.

\section{MC as bioaccumulative compounds in a regulatory context}

Bioaccumulation plays a vital role in understanding the fate of a substance in the environment. Substances having a high bioaccumulation potential can be efficiently taken up by organisms, leading to elevated internal concentrations that can reach critical levels that elicit toxic effects even when the concentration in the environment is low. Bioaccumulative chemicals are typically retained in the organism for a long period after the external concentration has declined. A chemical is considered "bioaccumulative" in the regulatory context of the Stockholm Convention mostly based on its potential to bioconcentrate (i.e., when its bioaccumulation factor in aquatic 
species is greater than $5000 \mathrm{ml} \mathrm{g}^{-1}$ wet weight or -- in the absence of such data -- when the log $\mathrm{K}_{\mathrm{OW}}$ is greater than 5) (Gobas et al. 2009). An overview of regulatory bioaccumulation assessment endpoints and criteria is provided in Gobas et al. 2009. Explosive MC compounds are not considered bioaccumulative compounds as their BCF values are $<14 \mathrm{ml} \mathrm{g}^{-1}$, therefore over two orders of magnitude lower than $5000 \mathrm{ml} \mathrm{g}^{-1}$.

\section{Toxicokinetics and biotransformation}

Direct uptake from water is usually the major process by which fish and aquatic invertebrates accumulate nonpolar organic compounds with low hydrophobicity, such as TNT and RDX. The bioaccumulation kinetics of TNT, 2-Am-DNT, 4-Am-DNT, and 2,4-DANT were compared in T. tubifex (Conder et al. 2004b) with all four compounds reaching steady state concentrations within $1 \mathrm{~h}$ of exposure. The uptake clearance rate coefficient $\left(k_{\mathrm{u}}\right)$ is defined as the volume or mass of a compartment scavenged of the contaminant per mass of organism per time. Using total radioactivity as a surrogate for whole body concentration, the $k_{\mathrm{u}}$ for TNT into the midge Chironomus dilutus, the oligochaete Lumbriculus variegatus (Belden et al. 2005a) and juvenile channel catfish (Ictalurus punctatus) (Belden et al. 2005a, Ownby et al. 2005) were $26.7,39.2$, and $10.1 \mathrm{ml} \mathrm{g}^{-1} \mathrm{~h}^{-1}$, respectively. Using analytically measured body residues, the $k_{\mathrm{u}}$ for TNT, 2-Am-DNT and 2,4-DANT into juvenile sheepshead minnows were $7.3,12.6$, and $1.3 \mathrm{ml} \mathrm{g}^{-1}$ $\mathrm{h}^{-1}$, respectively (Lotufo and Lydy 2005) and was $0.9 \mathrm{ml} \mathrm{g}^{-1} \mathrm{~h}^{-1}$ into adult mussels (Rosen and Lotufo 2007b). The rate of RDX uptake in sheepshead minnows (Lotufo and Lydy 2005), juvenile channel catfish (Belden et al. 2005b), L. variegatus (Belden et al. 2005b), and adult mussels (Rosen and Lotufo 2007b) were more variable $\left(0.15,1.3,5.2\right.$, and $3.4 \mathrm{ml} \mathrm{g}^{-1} \mathrm{~h}^{-1}$, respectively), but were consistently less efficient than the uptake for TNT. The uptake clearance rate for 2,4-DNT in carp (Cyprinus carpio) $\left(0.41 \mathrm{ml} \mathrm{g}^{-1} \mathrm{~h}^{-1}\right)$ (Lang et al. 1997) was slower than the rates derived for TNT using juvenile channel catfish or sheepshead minnows and may be due to species-specific differences and/or a decrease in uptake efficiency with increasing organism size, because the carp (5-18 g) were substantially larger than the juvenile minnows and catfish (0.1-0.4 g) used in the latter studies. The $k_{\mathrm{u}}$ for HMX was $0.06 \mathrm{ml} \mathrm{g}^{-1} \mathrm{~h}^{-1}$ for sheepshead minnows (Lotufo and Lydy 2005) and $0.6 \mathrm{ml} \mathrm{g}^{-1} \mathrm{~h}^{-1}$ for for adult mussels (Rosen and Lotufo 2007b) and was therefore slower than the $k_{\mathrm{u}}$ values determined for RDX for those organisms. Table 6.3 summarizes available uptake kinetics data for MC. The overall low efficiency of uptake of MC from water was expected because of the low hydrophobicity of these compounds. 
Table 6.3. Toxicokinetic model parameters for fish and aquatic invertebrates exposed to TNT, 2-Am-DNT, 2,4-DANT, 4-DNT, RDX and HMX. See Table 5.2 for species habitat information.

\begin{tabular}{|c|c|c|c|c|c|c|c|c|c|c|}
\hline Parameter & Species & Habitat & TNT & SumTNT & $\begin{array}{l}\text { 2-Am- } \\
\text { DNT }\end{array}$ & $\begin{array}{l}2,4- \\
\text { DANT }\end{array}$ & $\begin{array}{l}2,4- \\
\text { DNT }\end{array}$ & RDX & HMX & Reference \\
\hline \multirow{7}{*}{$\begin{array}{l}\text { Uptake } \\
\mathrm{k}_{\mathrm{u}} \\
\left(\mathrm{ml} \mathrm{g}^{-1} \mathrm{~h}^{-1}\right)\end{array}$} & $\begin{array}{l}\text { Sheepshead } \\
\text { minnow } \\
\text { Cyprinodon } \\
\text { variegatus }\end{array}$ & Marine & 7.3 & ND & 12.6 & 1.3 & ND & 0.15 & 0.06 & $\begin{array}{l}\text { Lotufo and Lydy } \\
2005\end{array}$ \\
\hline & $\begin{array}{l}\text { Fathead minnow } \\
\text { Pimephales } \\
\text { promelas }\end{array}$ & Freshwater & ND & 30.4 & ND & ND & ND & ND & ND & Yoo et al. 2006 \\
\hline & $\begin{array}{l}\text { Channel catfish } \\
\text { Ictalurus } \\
\text { punctuatus }\end{array}$ & Freshwater & 10.1 & ND & ND & ND & ND & 1.3 & ND & $\begin{array}{l}\text { Ownby et al. } \\
\text { 2005; Belden } \\
\text { et al. 2005b }\end{array}$ \\
\hline & $\begin{array}{l}\text { Mussel } \\
\text { Mytilus } \\
\text { galloprovincialis }\end{array}$ & Marine & 0.9 & 2.5 & ND & ND & ND & 3.4 & 0.6 & $\begin{array}{l}\text { Rosen and } \\
\text { Lotufo 2007b }\end{array}$ \\
\hline & $\begin{array}{l}\text { Midge } \\
\text { Chironomus } \\
\text { dilutus }\end{array}$ & Freshwater & 26.7 & ND & ND & ND & ND & ND & ND & $\begin{array}{l}\text { Belden et al. } \\
2005 a\end{array}$ \\
\hline & $\begin{array}{l}\text { Oligochaete } \\
\text { Lumbriculus } \\
\text { variegatus }\end{array}$ & Freshwater & 39.2 & ND & ND & ND & ND & 5.2 & ND & $\begin{array}{l}\text { Belden et al. } \\
2005 a, b\end{array}$ \\
\hline & $\begin{array}{l}\text { Common carp } \\
\text { Cyprinus carpio }\end{array}$ & Freshwater & ND & ND & ND & ND & 0.41 & ND & ND & $\begin{array}{l}\text { Lang et al. } \\
1997\end{array}$ \\
\hline \multirow{6}{*}{$\begin{array}{l}\text { Elimination } \\
\mathrm{ke}_{\mathrm{e}} \\
\left(\mathrm{h}^{-1}\right)\end{array}$} & $\begin{array}{l}\text { Cyprinodon } \\
\text { variegatus }\end{array}$ & Marine & 0.8 & ND & 1.0 & 2.7 & ND & 0.1 & 0.1 & $\begin{array}{l}\text { Lotufo and Lydy } \\
2005\end{array}$ \\
\hline & $\begin{array}{l}\text { Pimephales } \\
\text { promelas }\end{array}$ & Freshwater & ND & 2.2 & ND & ND & ND & ND & ND & Yoo et al. 2006 \\
\hline & $\begin{array}{l}\text { Ictalurus } \\
\text { punctuatus }\end{array}$ & Freshwater & 13.0 & ND & ND & ND & ND & 0.6 & ND & $\begin{array}{l}\text { Ownby et al. } \\
\text { 2005; Belden } \\
\text { et al. 2005b }\end{array}$ \\
\hline & $\begin{array}{l}\text { Mytilus } \\
\text { galloprovincialis }\end{array}$ & Marine & 2.1 & 2.2 & ND & ND & ND & 2.5 & 0.5 & $\begin{array}{l}\text { Rosen and } \\
\text { Lotufo 2007b }\end{array}$ \\
\hline & $\begin{array}{l}\text { Lumbriculus } \\
\text { variegatus }\end{array}$ & Freshwater & ND & ND & ND & ND & ND & 2.5 & ND & $\begin{array}{l}\text { Belden et al. } \\
2005 b\end{array}$ \\
\hline & $\begin{array}{l}\text { Oligochaete } \\
\text { Tubifex tubifex }\end{array}$ & Freshwater & ND & ND & 1.1 & ND & ND & ND & ND & $\begin{array}{l}\text { Conder et al. } \\
2004 c\end{array}$ \\
\hline \multirow{2}{*}{$\begin{array}{l}\text { Half-life } \\
t_{1 / 2 p}(h)\end{array}$} & $\begin{array}{l}\text { Cyprinodon } \\
\text { variegatus }\end{array}$ & Marine & 0.91 & ND & 0.72 & 0.25 & ND & 7.7 & 5.8 & $\begin{array}{l}\text { Lotufo and Lydy } \\
2005\end{array}$ \\
\hline & $\begin{array}{l}\text { Pimephales } \\
\text { promelas }\end{array}$ & Freshwater & ND & 0.31 & ND & ND & ND & ND & ND & Yoo et al. 2006 \\
\hline
\end{tabular}




\begin{tabular}{|l|l|l|l|l|l|l|l|l|l|l|}
\hline Parameter & Species & Habitat & TNT & SumTNT & $\begin{array}{l}\text { 2-Am- } \\
\text { DNT }\end{array}$ & $\begin{array}{l}\text { 2,4- } \\
\text { DANT }\end{array}$ & $\begin{array}{l}\text { 2,4- } \\
\text { DNT }\end{array}$ & RDX & HMX & Reference \\
\hline & $\begin{array}{l}\text { Ictalurus } \\
\text { punctuatus }\end{array}$ & Freshwater & 0.05 & ND & ND & ND & ND & 1.2 & ND & $\begin{array}{l}\text { Ownby et al. } \\
\text { 2005; Belden } \\
\text { et al. 2005b }\end{array}$ \\
\cline { 2 - 8 } & $\begin{array}{l}\text { Mytilus } \\
\text { galloprovincialis }\end{array}$ & Marine & 0.33 & 0.32 & ND & ND & ND & 0.27 & 1.50 & $\begin{array}{l}\text { Rosen and } \\
\text { Lotufo 2007b }\end{array}$ \\
\hline
\end{tabular}

ND - Not determined.

Biotransformation of TNT to Am-DNTs during aqueous exposures occurred very quickly in T. tubifex (0.212 and $0.187 \mathrm{~h}^{-1}$ for 2-Am-DNT and 4-AmDNT, respectively) (Conder et al. 2004b), but was markedly slower (0.06 h-1 for Am-DNTs) in juvenile sheepshead minnows (Lotufo and Lydy 2005). Substantial biotransformation of TNT to Am-DNTs was also reported for fathead minnows (Yoo et al. 2006) and adult mussels (Rosen and Lotufo $2007 \mathrm{~b}$ ) exposed to TNT for periods as short as 10 minutes. Formation of DANTs resulting from exposure to TNT or Am-DNTs was non-quantifiable or minimal (Lotufo and Lydy 2005, Conder et al. 2004b; Rosen and Lotufo 2007b).

Elimination of TNT and its major biotransformation products was relatively fast for all of the aquatic organisms tested. For example, when T. tubifex or sheepshead minnows were transferred to noncontaminated water following exposure to TNT, 2-Am-DNT, 4-Am-DNT ( T. tubifex only), or 2,4-DANT, measurable amounts of those compounds were completely eliminated from the tissues in less than $3 \mathrm{~h}$ (Lotufo and Lydy 2005; Conder et al. 2004b). The tissue sampling schedule used in the T. tubifex study precluded the calculation of the exceedingly fast elimination of TNT or 2,4-DANT in that species (Conder et al. 2004c). The elimination rate $\left(k_{\mathrm{e}}\right)$ for sumTNT was identical in juvenile fathead minnows and in adult mussels $\left(2.2 \mathrm{~h}^{-1}\right)$, corresponding to a half-life of $0.3 \mathrm{~h}$. The $k_{\mathrm{e}}$ of TNT in juvenile sheepshead minnows was slower, $0.8 \mathrm{~h}^{-1}$, corresponding to a half-life of $0.91 \mathrm{~h}$ (Lotufo and Lydy 2005). The $k_{\mathrm{e}}$ values for $T$. tubifex for 2-Am-DNTand 4-Am-DNT were $1.1 \mathrm{~h}^{-1}$ and $0.91 \mathrm{~h}^{-1}$, respectively (Conder et al. 2004b). For sheepshead minnows, the $k_{\mathrm{e}}$ for 2-Am-DNT and 2,4-DANT were 1.0 and $2.7 \mathrm{~h}^{-1}$, respectively (Lotufo and Lydy 2005). The rates of elimination of DNTs in goldfish (Wang et al. 1999) were very similar (0.47 and $0.60 \mathrm{~h}^{-1}$ for 2,4-DNT and 2,6-DNT, respectively) to the rates determined for TNT and 2-Am-DNT in T. tubifex and sheepshead minnows, as expected given the similar hydrophobicity of those nitroaromatic compounds. However, the rate of elimination of 2,4-DNT in carp was substantially slower (0.06 $\left.\mathrm{h}^{-1}\right)$, likely 
due to the larger size of the $C$. carpio compared to the organisms used in the other investigations. Elimination of RDX was relatively fast in juvenile channel catfish, adult mussels, and in the oligochaete L. variegatus (o.6, 2.5, and 2.5 $\mathrm{h}^{-1}$, respectively) (Belden et al. 2005b; Rosen and Lotufo 2007a), but was substantially slower in juvenile sheepshead minnows $\left(0.1 \mathrm{~h}^{-1}\right)$ (Lotufo and Lydy 2005). The $k_{\mathrm{e}}$ for HMX, reported for sheepshead minnows $\left(0.1 \mathrm{~h}^{-1}\right)$ and adult mussels $\left(0.5 \mathrm{~h}^{-1}\right)$ (Lotufo and Lydy 2005; Rosen and Lotufo 2007b) was very low.

\section{Formation and Elimination of TNT Bound Residues in Marine Invertebrates and Fish}

In the studies listed in Table 6.1, BCFs were determined using the parent compound concentrations determined by high performance liquid chromatography (HPLC) analysis of solvent extracts of whole-body homogenates. In early studies of the bioconcentration of explosives in aquatic invertebrates and fish, BCFs were derived using radioactivity measurements of whole-body homogenates as a surrogate for the sum molar concentration of parent and all transformation products. BCFs derived using total radioactivity measurements for body residues were reported for TNT, 2,4-DNT (Liu et al. 1983a), and RDX (Liu et al. 1983c) (Table 6.2). Recent studies of the bioconcentration of TNT parent compound in aquatic invertebrates and fish compared the BCFs calculated for the parent compound with BCFs calculated using the sum concentration of TNT parent compound and its transformation products in the tissues (Table 6.2).

A comparison of $\mathrm{BCF}$ for parent compounds, for total extractable compounds (radioactivity in the solvent extract) and for total extractable and non-extractable compounds (total radioactivity in the organism) for the same exposed organisms (Figure 6.1) reveals that the non-extractable (i.e., solvent-resistant and presumably tissue-bound) compounds are accumulated at substantially higher concentrations than extractable transformation products at steady state in fish and aquatic invertebrates. The tissue concentration of parent compounds represented only a fraction, sometimes minimal, of the total extractable concentration in all the species investigated.

Bioconcentration values determined using total radioactivity in the tissue were also reported for 2,4-DNT (Liu et al. 1983a) and RDX (Liu et al. 1983c) (Table 6.4). A comparison of BCFs determined for total radioactivity (Table 6.4) with those determined for a parent compound only (Table 6.1) 
for RDX suggests dominant accumulation of parent compound relative to transformation products in aquatic animals. A similar comparison for 2,4DNT suggests substantial accumulation of transformation products of that compound in aquatic animals.

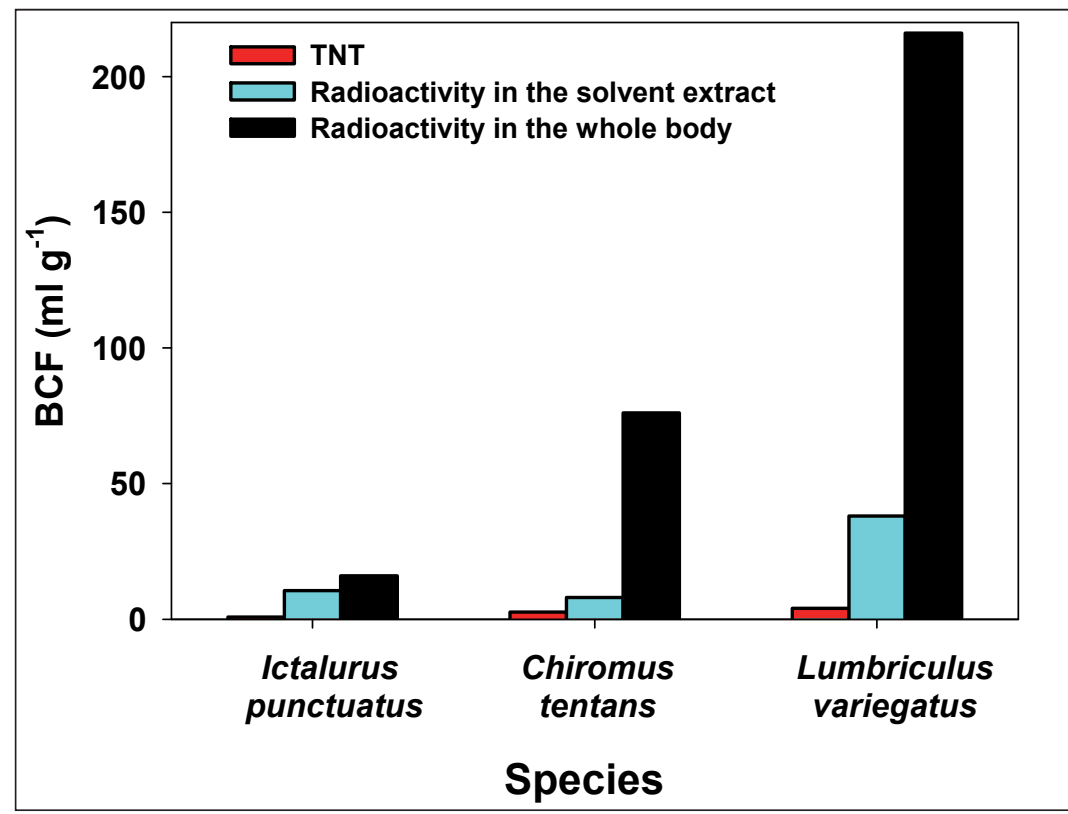

Figure 6.1. Bioconcentration factors (BCF) for freshwater fish and invertebrates exposed to TNT determined for body residues for parent compound, sum concentration of extractable compounds and for the sum concentration of extractable and non-extractable compounds (from

Lotufo et al. 2009b).

Table 6.4. Bioconcentration factor (BCF) values for aquatic animals determined using the sum concentration of the parent compound and transformation products obtained from radioactivity in the extract or whole body as surrogate for body residue.

\begin{tabular}{|c|c|c|c|c|c|}
\hline MC & Species & $\begin{array}{l}\text { Exposure } \\
\text { duration (d) }\end{array}$ & \begin{tabular}{|l}
$\mathrm{BCF}$ \\
$(\mathrm{ml} \mathrm{g-1)}$
\end{tabular} & $\begin{array}{l}\text { Analytical } \\
\text { measurement }\end{array}$ & Reference \\
\hline \multirow{6}{*}{ TNT } & \multirow{2}{*}{$\begin{array}{l}\text { Midge (larva) } \\
\text { Chironomus dilutus }\end{array}$} & 1 & 76 & $\begin{array}{l}\text { Radioactivity in the } \\
\text { whole body }\end{array}$ & Belden et al. 2005b \\
\hline & & 1 & 8 & $\begin{array}{l}\text { Radioactivity in the } \\
\text { solvent extract }\end{array}$ & Belden et al. 2005b \\
\hline & $\begin{array}{l}\text { Cladoceran } \\
\text { Daphnia magna }\end{array}$ & 4 & 209 & $\begin{array}{l}\text { Radioactivity in the } \\
\text { whole body }\end{array}$ & Liu et al. $1983 c$ \\
\hline & \multirow{3}{*}{$\begin{array}{l}\text { Oligochaete } \\
\text { Lumbriculus } \\
\text { variegatus }\end{array}$} & 4 & 202 & $\begin{array}{l}\text { Radioactivity in the } \\
\text { whole body }\end{array}$ & Liu et al. $1983 c$ \\
\hline & & 1 & 216 & $\begin{array}{l}\text { Radioactivity in the } \\
\text { whole body }\end{array}$ & Belden et al. 2005b \\
\hline & & 1 & 38 & $\begin{array}{l}\text { Radioactivity in the } \\
\text { solvent extract }\end{array}$ & Belden et al. 2005b \\
\hline
\end{tabular}




\begin{tabular}{|l|l|l|l|l|l|}
\hline \multirow{2}{*}{ MC } & Species & $\begin{array}{l}\text { Exposure } \\
\text { duration }(\mathrm{d})\end{array}$ & $\begin{array}{l}\text { BCF } \\
\left(\mathrm{ml} \mathrm{g} \mathbf{g}^{-1}\right)\end{array}$ & $\begin{array}{l}\text { Analytical } \\
\text { measurement }\end{array}$ & Reference \\
\hline \multirow{2}{*}{$\begin{array}{l}\text { Channel catfish } \\
\text { Ictalurus punctuatus }\end{array}$} & 0.3 & 16 & $\begin{array}{l}\text { Radioactivity in the } \\
\text { whole body }\end{array}$ & Ownby et al. 2005 \\
\cline { 3 - 7 } & 0.3 & 10.5 & $\begin{array}{l}\text { Radioactivity in the } \\
\text { solvent extract }\end{array}$ & Ownby et al. 2005 \\
\hline \multirow{2}{*}{ RDX } & Daphnia magna & 4 & 13 & $\begin{array}{l}\text { Radioactivity in the } \\
\text { whole body }\end{array}$ & Liu et al. 1983a \\
\cline { 2 - 7 } & $\begin{array}{l}\text { Lumbriculus } \\
\text { variegatus }\end{array}$ & 4 & 58 & $\begin{array}{l}\text { Radioactivity in the } \\
\text { whole body }\end{array}$ & Liu et al. 1983a \\
\cline { 2 - 7 } & $\begin{array}{l}\text { Daphnia magna } \\
\text { Lumbriculus }\end{array}$ & 4 & 3 & $\begin{array}{l}\text { Radioactivity in the } \\
\text { whole body }\end{array}$ & Liu et al. 1983c \\
variegatus & 4 & 1.6 & $\begin{array}{l}\text { Radioactivity in the } \\
\text { whole body }\end{array}$ & Liu et al. 1983c \\
\hline
\end{tabular}

Bioaccumulation of large fractions of non-identified compounds was also found in the marine amphipod Eohaustorius estuarius following exposure to sediment spiked with (14C)TNT (Rosen and Lotufo 2005). For RDX, comparisons of the steady state BCF reported for total radioactivity $(3.0 \mathrm{ml}$ $\mathrm{g}^{-1}$ ) (Liu et al. 1983c) and parent compound ( $\left.2.4 \mathrm{ml} \mathrm{g}^{-1}\right)$ for L. variegatus suggests that minimal biotransformation of RDX occurs in that species.

Non-extractable radioactivity in aquatic invertebrates and fish tissues likely represents covalent conjugates (i.e., macromolecular bound residues) (Conder et al. 2004b, Belden et al. 2005b). However, neither the biological half-life nor the chemical nature of non-extractable transformation products in invertebrates and fish has been investigated to date.

The formation of covalent bonds with proteins in mammalian systems has been reported (Liu et al. 1992; Leung et al. 1995; Bakhtiar et al. 1997). A recent investigation of ${ }^{14} \mathrm{C}-\mathrm{TNT}$ toxicokinetics in earthworms (Belden et al. 2011) showed that nonextractable residues accounted for as much as $68 \%$ of total body burden. Parent TNT accounted for less than $3 \%$ of radioactivity, while Am-DNTs accounted for 7 to $38 \%$. Elimination was much slower for nonextractable residues. The half-lives were 61 to 120 hours for TNT, AmDNTs, and DANTs, which was significantly lower than the half-lives found for nonextractable residues, which ranged from 201 to 240 hours. Despite resisting organic solvent extraction, $80 \%$ of the nonextractable residue in earthworms was solubilized when residual tissue was treated with a dilute aqueous solution of hydrochloric acid (Belden et al. 2011). This extraction behavior is consistent with the assumed transformation pathway shown in Figure 6.2. That is, polar conjugates formed between reactive TNT 
metabolites and proteins (e.g., the sulfonamide conjugate shown in Figure 6.2) would not be expected to be solubilized by organic solvents. On the other hand, treatment with diluted acid could feasibly break the S-N bond responsible for conjugation and release the TNT-derived portion of the molecule (i.e., reductive metabolites) into aqueous solution.

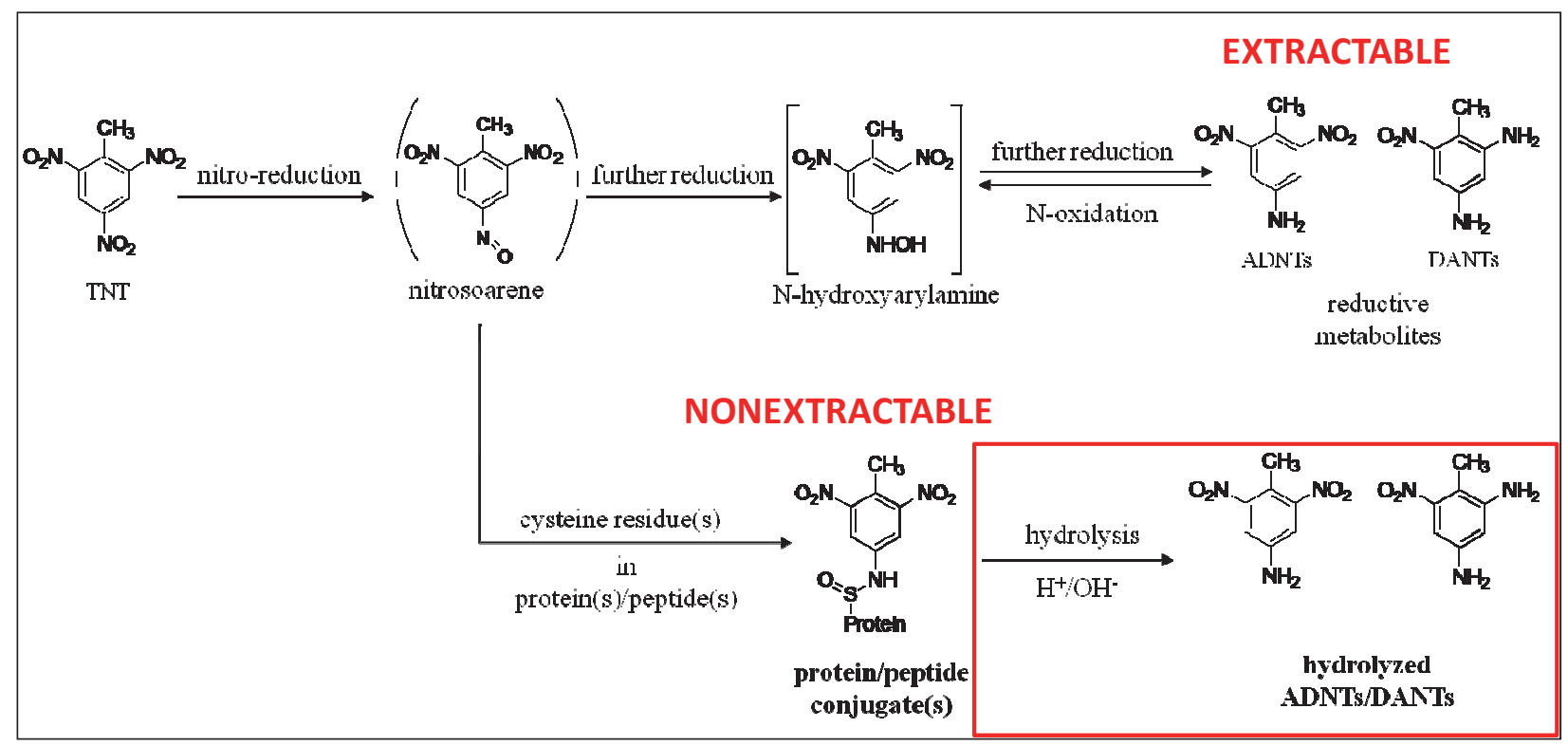

Figure 6.2. Proposed metabolic activation and formation of protein-bound TNT residues in fish and aquatic invertebrates. The red box indicates the identification of Am-DNTs and DANTs as hydrolysable bound residues in tissue of TNT-exposed earthworms (Belden et al. 2011).

Lotufo et al. (unpublished study) examined ${ }^{14} \mathrm{C}-\mathrm{TNT}$ toxicokinetics of juvenile sheepshead minnows and adult mussels exposed to $0.3 \mathrm{mg} \mathrm{L}^{-1}$ TNT in seawater. After $24 \mathrm{~h}$, mean BCFs were $4 \mathrm{ml} \mathrm{g}^{-1}$ and $1.3 \mathrm{ml} \mathrm{g}^{-1}$ for fish and mussels, respectively. Those values are similar to those derived from kinetics exposures reported in published studies (Table 6.1).

Based on the concentration of Am-DNTs (present in fish and mussels) and DANTs (present in fish only) in tissues following aqueous exposure, it is apparent that TNT was efficiently biotransformed. The body residues for TNT, Am-DNTs, and DANTs corresponded to 29, 18 and 53\% of the total sum TNT residue, respectively, for fish and 18, 82 and o\% for mussels. The use of radiolabeled TNT allowed determination of the concentration of unknown extractable products (i.e., polar transformation products, not Am-DNTs and DANTs), as well as non-extractable residues as sum concentration (i.e., without identification and quantification of individual compounds), as described in Section 6. Considering only the extractable parent and transformation products, polar unknown compounds 
accounted for most of the bioaccumulation in TNT-exposed fish and mussels, while the TNT parent compound was present as only to $12 \%$ and $7 \%$ of the total accumulation in fish and mussels, respectively (Table 6.5). When the radioactivity remaining in tissue homogenate following solvent extraction was considered, non-extractable compounds accounted for $83 \%$ and $75 \%$ of the total accumulation in fish and mussels, respectively, while Sum-TNT corresponded to only 7 and 10\%, respectively (Table 6.6).

Table 6.5. Percentage of total body residue for solvent extractable compounds in fish and mussels (from Lotufo et al. unpublished study).

\begin{tabular}{|l|l|l|}
\hline Species & Compound / Group & Percent of Total Body Residue \\
\hline \multirow{4}{*}{$\begin{array}{l}\text { Sheepshead } \\
\text { minnow } \\
\begin{array}{l}\text { Cyprinodon } \\
\text { variegatus }\end{array}\end{array}$} & TNT & 11.9 \\
\cline { 2 - 3 } & Am-DNTs & 7.4 \\
\cline { 2 - 3 } & DANTs & 22.1 \\
\cline { 2 - 3 } & SumTNT & 41.3 \\
\cline { 2 - 3 } & Extractable by acetonitrile - unknown & 58.7 \\
\hline \multirow{4}{*}{$\begin{array}{l}\text { Mussel } \\
\text { gallilus }\end{array}$} & TNT & 7.3 \\
\cline { 2 - 3 } & Am-DNTs & 32.6 \\
\cline { 2 - 3 } & DANTs & 0 \\
\cline { 2 - 3 } & SumTNT & 39.9 \\
\cline { 2 - 3 } & Extractable by acetonitrile - unknown & 60.1 \\
\hline
\end{tabular}

Table 6.6. Percentage of total body residue for solvent-extracted compounds and for bound (non-extractable) residues in fish and mussels exposed to radiolabeled TNT (from Lotufo et al. unpublished study).

\begin{tabular}{|c|c|c|}
\hline Species & Compound / Group & $\begin{array}{l}\text { Percent of Total Body } \\
\text { Residue }\end{array}$ \\
\hline \multirow{6}{*}{$\begin{array}{l}\text { Sheepshead } \\
\text { minnow } \\
\text { Cyprinodon } \\
\text { variegatus }\end{array}$} & TNT & 2.0 \\
\hline & Am-DNTs & 1.2 \\
\hline & DANTs & 3.7 \\
\hline & SumTNT & 6.8 \\
\hline & Extractable by acetonitrile - unknown & 9.7 \\
\hline & Non-extractable & 83.4 \\
\hline \multirow{6}{*}{$\begin{array}{l}\text { Mussel } \\
\text { Mytilus } \\
\text { galloprovincialis }\end{array}$} & TNT & 1.8 \\
\hline & Am-DNTs & 8.2 \\
\hline & DANTs & 0 \\
\hline & SumTNT & 10.0 \\
\hline & Extractable by acetonitrile - unknown & 15.1 \\
\hline & Non-extractable & 74.9 \\
\hline
\end{tabular}


Upon transfer of TNT-exposed fish and mussels to uncontaminated water, the persistence of extractable and nonextractable residues in tissues differed markedly. The half-life of sumTNT in fish and mussels was only 5.5 and $27 \mathrm{~h}$, respectively (Table 6.7). In contrast, the half-lives for non-extractable residues was 80 and $325 \mathrm{~h}$ for fish and mussels, respectively (Table 6.7), indicating the substantially higher persistence of bound residues.

Table 6.7. Mean elimination half-lives for SumTNT and for nonextractable residues in fish and mussels (from Lotufo et al. unpublished study).

\begin{tabular}{|c|c|c|}
\hline Species & Compound / Group & Half-life $(h)^{a}$ \\
\hline \multirow{2}{*}{$\begin{array}{l}\text { Sheepshead } \\
\text { minnow } \\
\text { Cyprinodon } \\
\text { variegatus }\end{array}$} & SumTNT & 5.5 \\
\hline & Nonextractable & 80 \\
\hline \multirow{2}{*}{$\begin{array}{l}\text { Mussel } \\
\text { Mytilus } \\
\text { galloprovincialis }\end{array}$} & SumTNT & 27 \\
\hline & Nonextractable & 325 \\
\hline
\end{tabular}

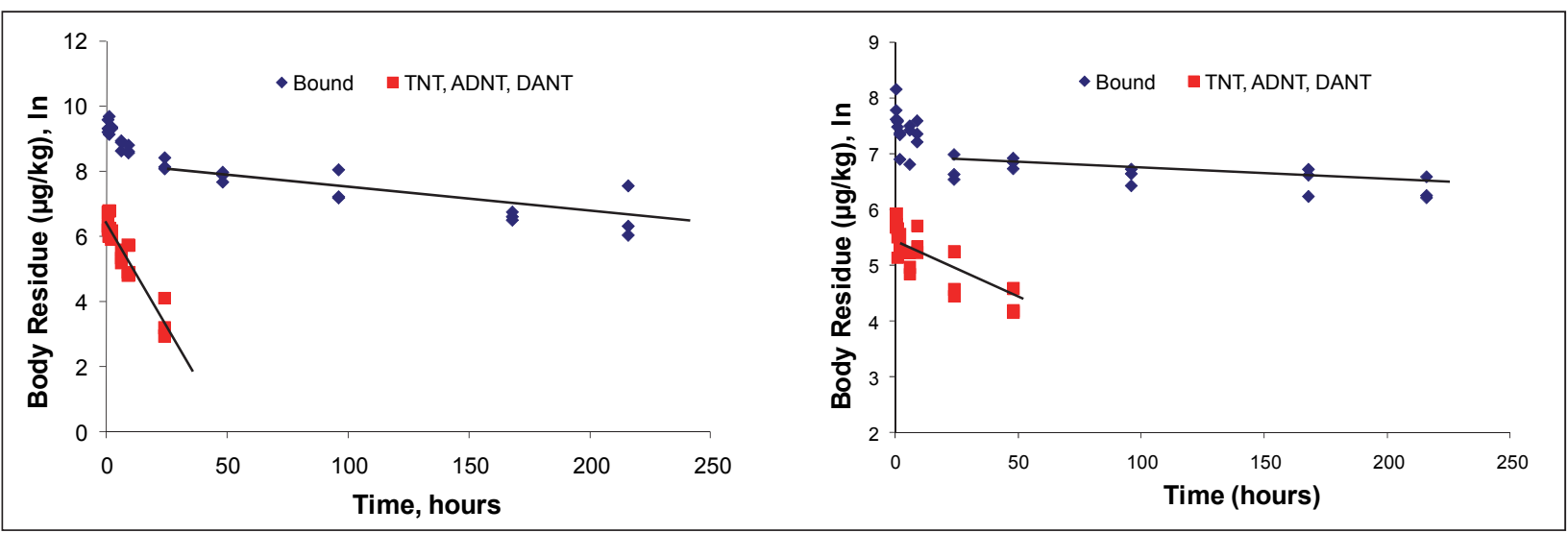

Figure 6.3. Depuration regressions for sheepshead minnows (left) or mussels (right) exposed to ${ }^{14} \mathrm{C}-\mathrm{TNT}$-spiked seawater (Lotufo et al. unpublished study). Red squares are used for sum of TNT and major aminated transformation products and blue diamonds are used for nonextractable (bound) residues. Regression calculations exclude time points prior to $12 \mathrm{~h}$ for bound residues.

\section{Bodypart-specific bioaccumulation}

Sheepshead minnows were exposed to radiolabeled isotopes of TNT, RDX and HMX to assess the body distribution of those compounds (Lotufo 2011). To allow a comprehensive understanding of the biological fate of those explosives in fish, tissue concentrations were determined for parent compound and major breakdown products. Both solvent-extractable and solvent-nonextractable radiolabeled transformation products were evaluated for mass-balance purposes. The relative contribution of the different body parts assessed in this study to the total fish biomass is 
presented in Table 6.8. The contribution of the amount of explosive parent compound or transformation products in a body part relative to the total amount present in the fish is also presented in Table 6.8. Although the liver accounted for a very small fraction of the total biomass, most of the TNT transformation products resided in that organ. Contrastingly, most of the TNT parent compound was present in the viscera, which also accounted for a small fraction of the total biomass (Table 6.8). The remaining tissues (muscles, skin, and subdermal fat) contained a disproportionately small fraction of the accumulated TNT parent compound and transformation products. The contribution of the liver RDX and HMX parent compound and transformation products was disproportionately high, but less so than for TNT; most of the RDX and HMX parent compound and transformation products were present in the body remains (Table 6.8).

Table 6.8. Contribution (as a percent) of different body parts to the total amount of bioaccumulated compounds for different classes of analytes in sheepshead minnows exposed to TNT, RDX and HMX (from Lotufo 2011).

\begin{tabular}{|c|c|c|c|c|}
\hline & Liver & Viscera & Gills & Body remains \\
\hline & $3.0^{a}$ & $9.1^{a}$ & $2.1^{a}$ & $85.8^{a}$ \\
\hline \multicolumn{5}{|c|}{ TNT } \\
\hline Total & 45.6 & 22.1 & 1.7 & 30.6 \\
\hline Solvent-extracted & 36.3 & 32.3 & 2.0 & 29.4 \\
\hline TNT & 0.5 & 63.7 & 2.6 & 33.3 \\
\hline Am-DNTs & 63.1 & 18.7 & 4.2 & 14.0 \\
\hline \multicolumn{5}{|c|}{ RDX } \\
\hline Total & 8.4 & 13.4 & 5.7 & 72.5 \\
\hline Solvent-extracted & 6.1 & 15.6 & 5.2 & 73.1 \\
\hline RDX & 5.0 & 26.3 & 8.7 & 60.1 \\
\hline \multicolumn{5}{|c|}{ HMX } \\
\hline Total & 11.5 & 13.3 & 6.6 & 68.6 \\
\hline Extracted & 11.9 & 17.4 & 7.7 & 63.0 \\
\hline
\end{tabular}

Total - percent of the total concentration of parent and all transformation products compounds in the body part, Solvent-extracted - acetonitrile-extracted compounds measured using radioactivity, a contribution (as percent) of each body part to the total fish biomass is also provided.

\section{Bioaccumulation from diet}

The dietary uptake of TNT and RDX was examined using a marine fish, sheepshead minnows (Lotufo and Blackburn et al. 2010), and two freshwater fish, channel catfish (Belden et al. 2005a, 2005b) and fathead 
minnow (Houston and Lotufo 2005) using different routes of contaminant delivery.

Juvenile channel catfish force-fed with ${ }^{14} \mathrm{C}-\mathrm{TNT}$-spiked food pellets daily for up to $10 \mathrm{~d}$ accumulated radioactivity, mostly as extractable and nonextractable unknown compounds. The ratio of the concentration in fish relative to the TNT concentration in the diet $\left(0.000024 \mathrm{~g} \mathrm{~g}^{-1}\right)$, to the sum of extractable compounds (0.00048 $\left.\mathrm{g} \mathrm{g}^{-1}\right)$, and to the total parent and transformation products (including covalently bound compounds) (0.00091 $\mathrm{g} \mathrm{g}^{-1}$ ) were exceedingly low (Belden et al. 2005a).

Juvenile sheepshead minnows were fed fresh prey (the amphipod Leptocheirus plumulosus) pre-exposed to ${ }^{14} \mathrm{C}-\mathrm{TNT}$. Fish received a meal twice daily for up to $7 \mathrm{~d}$ (Lotufo and Blackburn 2010). Fathead minnows were fed frozen prey (Lumbriculus variegatus worms) pre-exposed to ${ }^{14} \mathrm{C}$ TNT or ${ }^{14} \mathrm{C}-\mathrm{RDX}$. Fish received a meal twice daily for up to $14 \mathrm{~d}$ (Houston and Lotufo 2005). Those two studies used prey pre-exposed to ${ }^{14} \mathrm{C}$-dichlorodiphenyltrichloroethane ( $\left.{ }^{14} \mathrm{C}-\mathrm{DDT}\right)$ as a positive control for dietary uptake in the latter study because that compound is substantially more hydrophobic; hence, the compound will bioaccumulate to a greater extent than explosives. The body residues in the fish remained relatively unchanged over time for ${ }^{14} \mathrm{C}-\mathrm{TNT}$ and ${ }^{14} \mathrm{C}-\mathrm{RDX}$. The ratio of the concentration in fish relative to the concentration in the diet, determined using total radioactivity in worms and fathead minnows were 0.018 , 0.010 , and $0.422 \mathrm{~g} \mathrm{~g}^{-1}$ for ${ }^{14} \mathrm{C}-$

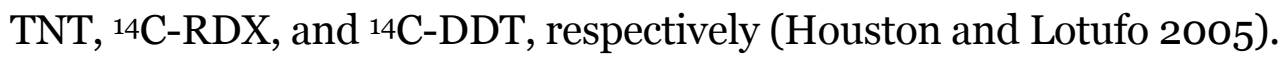
Those ratios, determined using total radioactivity in amphipods and sheepshead minnows were 0.068 and $0.388 \mathrm{~g} \mathrm{~g}^{-1}$ for ${ }^{14} \mathrm{C}$-TNT, and ${ }^{14} \mathrm{C}$-DDT, respectively (Lotufo and Blackburn et al. 2010). Those low ratios confirm the expected low bioaccumulative potential for TNT and RDX through the dietary route.

To directly assess the relative importance of aqueous and dietary uptake of $\mathrm{RDX}$, juvenile channel catfish were fed L. variegatus freshly-exposed to RDX, directly exposed to RDX-spiked water, or exposed to both, for $7 \mathrm{~d}$ (Belden et al. 2005b). The same concentration was used for the worm and fish aqueous exposures. The mean bioaccumulation factor (BAF) of $0.04 \mathrm{~g}$ $\mathrm{g}^{-1}$ obtained from that study for parent RDX was higher than the mean BAF (0.01 $\left.\mathrm{g} \mathrm{g}^{-1}\right)$ determined using radioactivity as a surrogate for RDX and its transformation products using fathead minnows (Houston et al. 2005). Results from the comparative exposure scenario study (Belden et al. 
$2005 \mathrm{~b}$ ) as well as from other dietary uptake investigations (Houston and Lotufo 2005; Belden et al. 2005a) indicated that aqueous exposure is likely the dominant route of exposure for TNT, RDX and similar compounds, with dietary uptake providing only minimal, if any, contribution to the net bioaccumulation of RDX in aquatic systems. 


\section{Fate, Toxicity, and Bioaccumulation of MC Released from Composition B under Realistic Exposure Conditions}

This section is a synopsis of information published in Rosen and Lotufo (2010).

It is anticipated that over time $\mathrm{MC}$ will leak from munitions in the underwater environment, resulting in the slow discharge of these chemicals into the surrounding water and sediment on a relatively localized scale. It is also possible that low-order detonations will create fragments of MC (e.g., Composition B) that allow subsequent biological exposure. Although the extent of such exposure is largely unknown, low concentrations of some MC compounds have been measured in marine sediment (Darrach et al. 1998; Pascoe et al. 2010). The objective of this study was to evaluate the fate of Composition B (a military formulation containing TNT and RDX) fragments, or "fragments," in aquaria containing seawater and sediment under several different exposure scenarios over a period of approximately one month. The approach was intended to more closely reflect potential real-world exposure scenarios compared to traditional laboratory experiments that involve fortifying water or sediment with pure compounds to create concentrations high enough to elicit effects and develop toxicity or bioaccumulation metrics.

One fish and four different invertebrate species were included in the experimental exposure to more closely simulate real world conditions (i.e., a microcosm) than single species studies would. The authors of this study also intended to more accurately assess any differences in toxicity and bioaccumulative potential among species. Sandy, low organic carbon sediment was used as substrate to minimize binding of compounds to sediment, resulting in a worst-case scenario of MC bioavailability to ecological receptors. The build up of $\mathrm{MC}$ released from the fragments into the sediment, porewater and overlying compartment; bioaccumulation; and effects to fish and invertebrates under different treatments were investigated. 


\section{Experimental Design}

A 3 by 2 factorial experimental design was used to investigate effects of Composition B placement ("Exposed" at sediment surface, "Buried," or no Composition B) and absence or presence of water exchange ("Static" vs. "Flow") on the concentration of MC in the overlying water, porewater, and uptake by organism tissues.

Experimental units were 20-L aquaria, which were replicated three times for each treatment type. An illustration of a typical tank setup is shown in Figure 7.1. Each tank received enough uncontaminated sandy sediment (Yaquina Bay, OR, USA; TOC $=<0.1 \%$ ) to yield a $3 \cdot 5-\mathrm{cm}$ thick layer. Cylindrical field exposure chambers were filled with $2 \mathrm{~cm}$ of sediment and gently pushed approximately $2 \mathrm{~cm}$ into the sediment so that animals could be added later through tubes extending above the water surface. Chambers were used for amphipods and polychaetes to ease their recovery from the aquaria, and to strategically control organism exposure. The chambers were positioned so that each would be approximately the same distance away from the Composition B fragments.

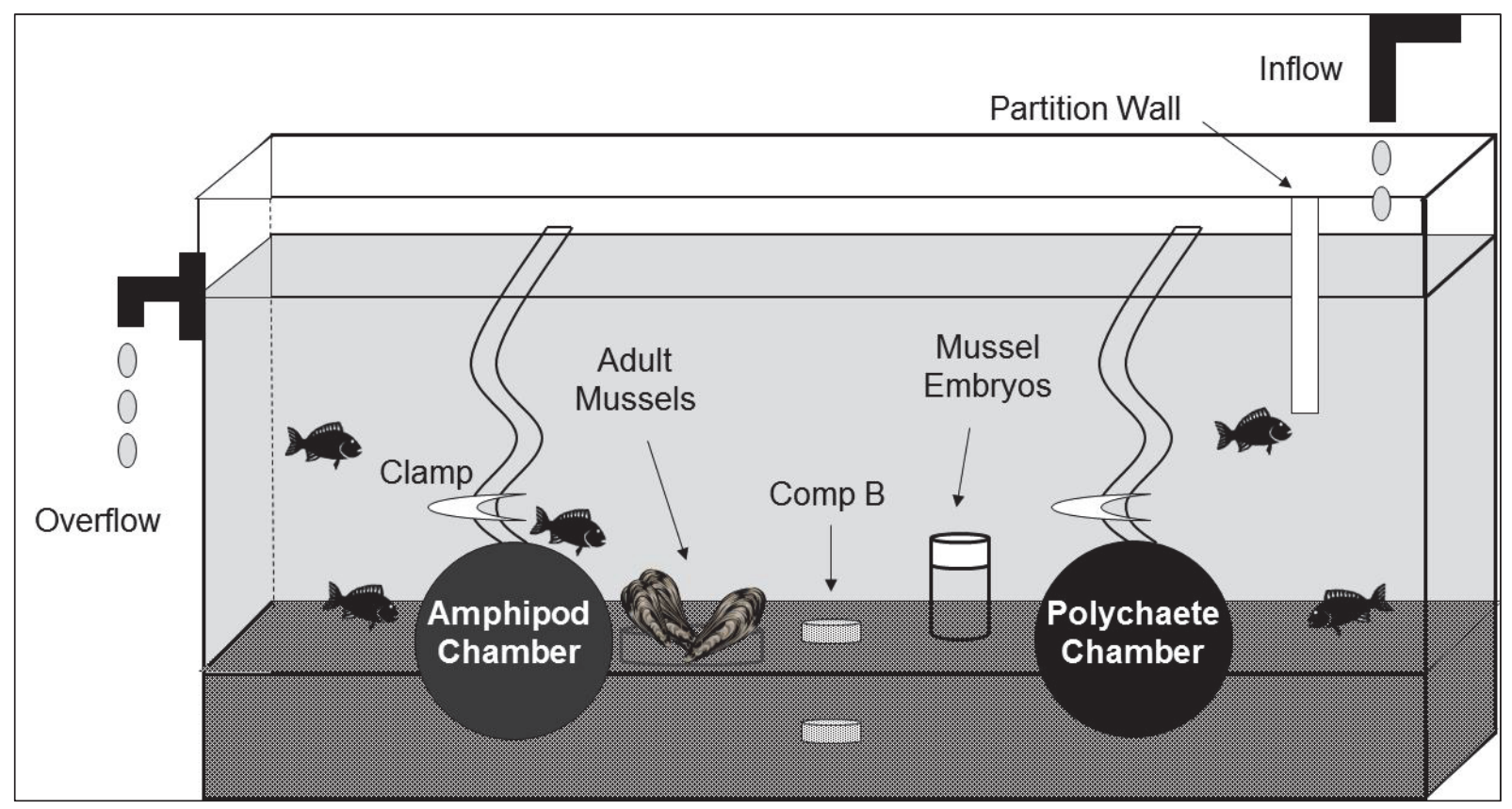

Figure 7.1. Exposure system used to assess fate and effects of Composition B.

Reconstituted seawater (Instant Ocean commercial salt mix in dechlorinated water) was gently added to the aquaria. Three Composition B exposure treatments were used: 1) "Exposed" at the sediment surface; 
2) "Buried" at a depth of approximately $2 \mathrm{~cm}$; and 3) "No Composition B" (control). Each Composition B treatment type was held under two different water exchange rates: 1) "Static," or 2) "Flow." The Flow treatment resulted in renewal of each tank with clean seawater at a rate of 0.5 turnovers per day ( 1 full exchange of overlying water every other day).

Except for control tanks, each tank received two Composition B fragments weighing a total of approximately $0.4 \mathrm{~g}$ (range $=0.39-0.41 \mathrm{~g}$ ). Composition B fragments consisted of 59.5\% RDX, 39.5\% TNT, and $1 \%$ wax by mass and were obtained from the Holston Army Ammunition Plant (Kingsport, TN). Fragments were placed immediately adjacent to one another on (Exposed) or in (Buried) the sediment approximately equidistantly from all sides of the tank. Under this exposure scenario, overlying water samples were removed every other day for a period of 24 days for MC concentration. Following this period, test organisms were added for up to an additional 10 days, making the entire experiment a total of 34 days.

After the 24-d fate characterization period, test organisms were added to the tanks. Amphipods and polychaetes, both sediment dwellers, were added to the cylindrical chambers with mesh screens. Fish were added and allowed to swim freely throughout the tank. Small adult mussels were positioned in a shallow glass dish at the sediment-water interface. In addition, mussel embryos were housed in an open glass scintillation vial placed on the sediment surface. All organisms were exposed for 10 days, except the embryonic mussels, which were exposed for $48 \mathrm{~h}$ only. Tissue concentrations for relevant test organisms were measured to determine bioconcentration factors.

The microcosms were maintained at $18^{\circ} \mathrm{C}$ with a $16: 8 \mathrm{~h}$ light: dark photoperiod. After 2 days, the embryonic mussels were removed from the tanks and assessed for normal development. After 10 days, all other test organisms were removed, assessed for survival and bioaccumulation. Porewater was sampled and analyzed upon experiment termination.

\section{MC in the overlying water}

At termination of the 34-d exposure period, approximately $15.5 \%$ of the initial Composition B mass had been lost from Exposed Composition B fragments, while only about $1.8 \%$ loss was observed from Buried Composition B fragments. Mean loss of fragment mass in both Exposed 
and Buried fragments was about 10\% greater in the presence of Flow, but this difference was not statistically significant.

The loss of fragment mass was largely attributable to dissolution into the overlying water. The concentration of SumTNT in the overlying water increased linearly in the Static Exposed (SE) treatment tanks for the first 10 days, and then leveled off for the remainder of the exposure (Figure 7.2). SumTNT concentration was highest in the SE treatment compared to any of the other three treatments. This was expected, as this was hypothesized to be the worst-case scenario treatment. Flow Exposed (FE) treatment tank TNT concentrations leveled off more quickly and were $62 \%$ lower than SE treatments, due to the loss of compounds through gradual water exchange. SumTNT was composed of $78 \%$ parent compound under Flow, but only 46\% parent TNT under Static conditions.

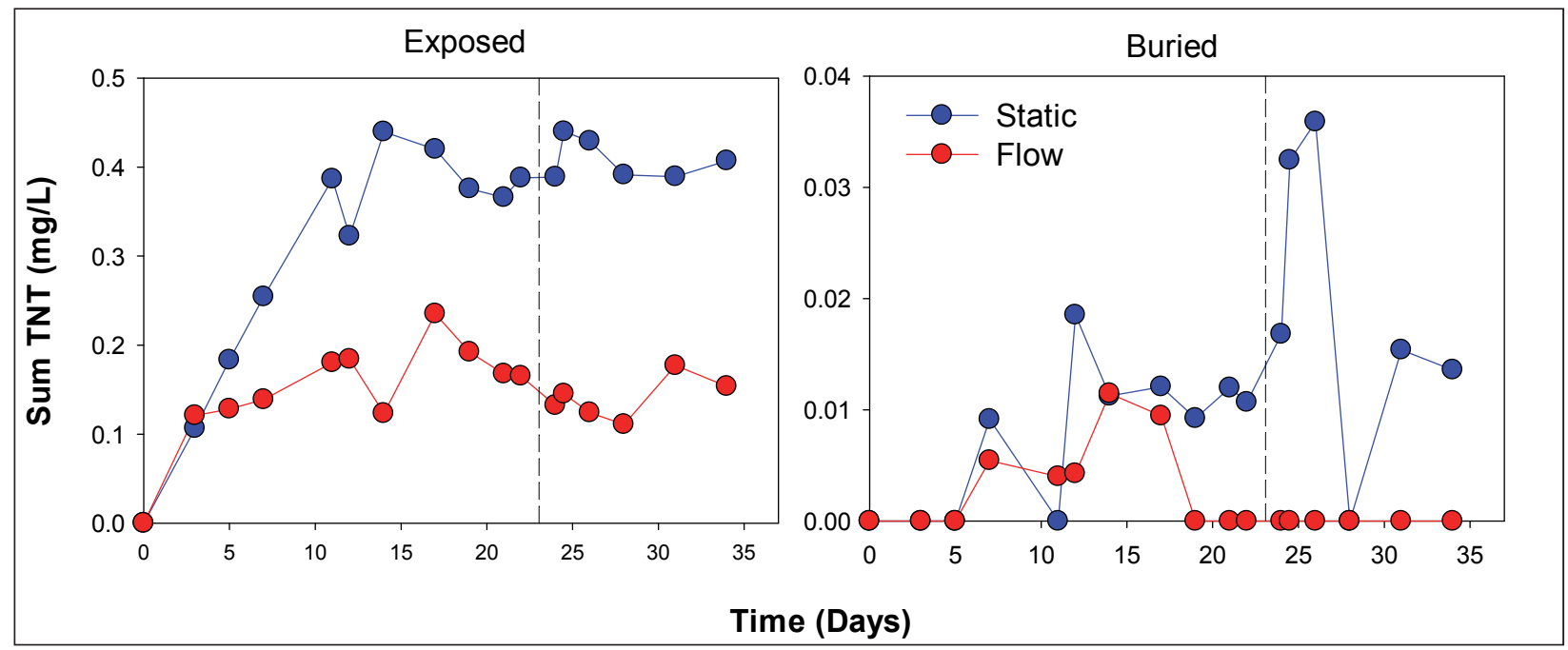

Figure 7.2. TNT concentration in the overlying water from exposures containing Composition B fragments under both static and flow conditions. Fragments were placed either at sediment surface ("Exposed"; left figure) or at a sediment depth of approximately $2 \mathrm{~cm}$ ("Buried"; right figure). Note the different scales for the $y$ axis. The vertical dashed line represents when test organisms were added (day 23).

RDX behaved similarly to TNT in that overlying water concentrations in Exposed tanks increased linearly for the first 10 days followed by stabilization under Static conditions, while steady-state conditions occurred much more quickly (by day 3) under Flow conditions (Figure 7.3). It is interesting to note that unlike TNT, RDX started to increase linearly again in the SE treatment following the addition of the test organisms (Figure 7.3). It is unclear why this occurred, but it could be associated with bioturbation around the fragment by the various test organisms as well as added circulation of water around the fragment associated with the addition of 
gentle aeration to the system upon the addition of organisms. As with TNT, RDX did not appreciably partition into the overlying water in treatments in which Composition B was buried.

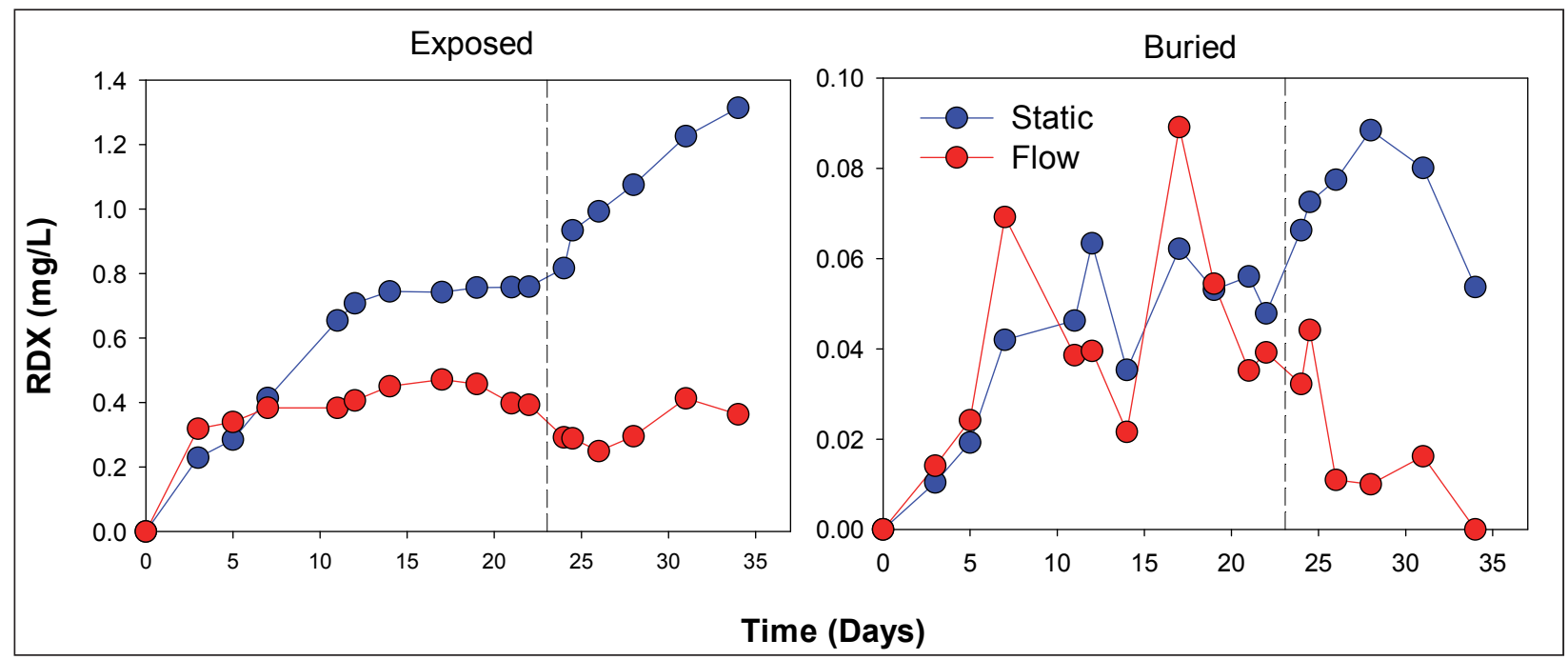

Figure 7.3. The concentration of RDX in the overlying water from exposures containing Composition B fragments, under both static and flow conditions. Fragments were either placed at sediment surface ("Exposed" left figure) or at a sediment depth of approximately $2 \mathrm{~cm}$ ("Buried"; right figure). Note the different scales for the y-axis. Vertical dashed line represents when test organisms were added (day 23).

Both TNT and RDX concentrations in the overlying water (up to 0.4 and $1.3 \mathrm{mg} \mathrm{L}^{-1}$, respectively) were substantially lower than the approximate expected solubility limit for those compounds ( $130 \mathrm{mg} \mathrm{L}^{-1}$ and $40 \mathrm{mg} \mathrm{L}^{-1}$ for TNT and RDX, respectively). While the Composition B fragment served as the source of RDX and TNT to the overlying water, volatilization, transformation to non-identified compounds, and some binding to sediment likely contributed towards the higher apparent rate of removal of TNT from that compartment. Based on the temporal trend for the compounds, increasing concentrations of RDX but stable concentrations of TNT would be predicted for the days or weeks following the 34-d exposure period used in this study.

\section{MC in the porewater}

SumTNT and RDX concentrations were always higher directly under the fragment, as compared to concentrations in the overlying water, but the difference was most dramatic for Static exposures, both when the Composition B fragment was Exposed and Buried (Figure 7.4). Porewater TNT and RDX concentrations under the fragment were as much as 1 order of magnitude higher than the overlying water concentrations in SE 


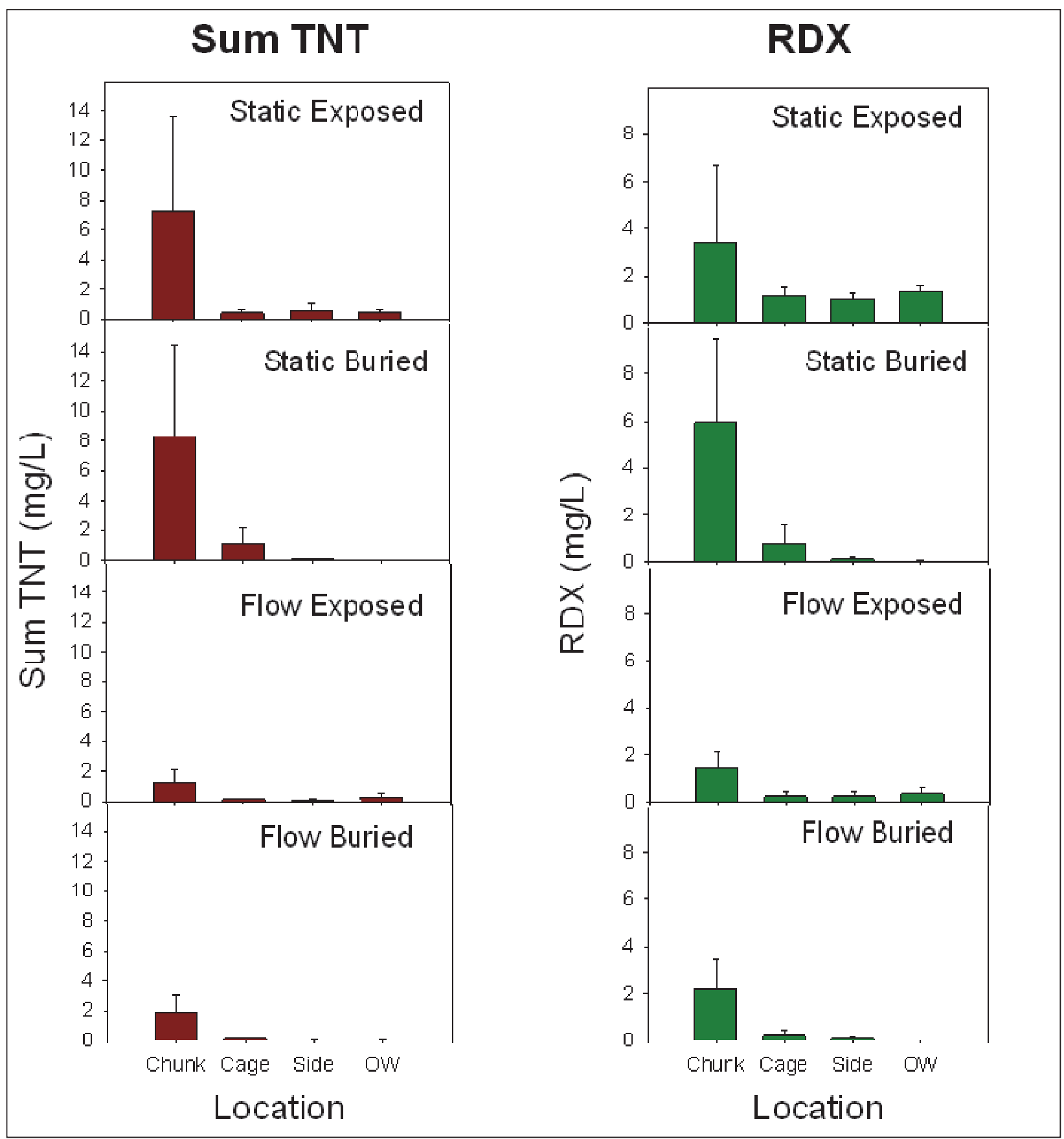

Figure 7.4. Mean ( \pm 1 standard deviation) SumTNT (left) and RDX (right) porewater concentrations at termination of exposures with Composition $B$ fragments. Porewater was sampled directly beneath the Composition B fragment ("Chunk") in the center of each tank, and at increasing distance away from the fragment, next to exposure chamber ("Cage"), and adjacent to edge of glass tank ("Side"). Overlying water (OW) concentrations are also shown for comparison.

treatments and up to over 2 orders of magnitude higher than the overlying water in the Static Buried treatments, yet still well below the solubility limits for these compounds. The potential for heightened exposure to organisms residing in the sediment, however, appears to be limited to the immediate 
vicinity to the fragment, as concentrations approached the relatively low overlying water concentrations just a few $\mathrm{cm}$ from the Composition $\mathrm{B}$ fragments (Figure 7.4). In addition, in the presence of Flow, porewater concentrations directly under the Comp B fragments were only minimally elevated relative to overlying water concentrations (Figure 7.4).

\section{Toxicity of MC released from Composition B}

No significant reductions in survival were observed for any test species relative to control tanks without Composition B fragments (Table 7.1). This was not unexpected, as water concentrations did not achieve previously reported lethal concentrations for amphipod, polychaete, minnow, or adult mussel (see Section 2). Somewhat reduced survival in some amphipod and polychaete treatments is likely related to issues associated with recovery from the field exposure chambers, as opposed to effects associated with contaminant exposure, as some reduced survival was also observed in the control treatments (Table 7.1).

Table 7.1. Test organism survival, and embryo-larval development success following exposures with Composition B fragments.

\begin{tabular}{|c|c|c|c|c|c|c|}
\hline Species & \begin{tabular}{|l} 
Static \\
Control
\end{tabular} & \begin{tabular}{|l} 
Flow \\
Control
\end{tabular} & $\begin{array}{l}\text { Static } \\
\text { Exposed }\end{array}$ & \begin{tabular}{|l} 
Flow \\
Exposed
\end{tabular} & $\begin{array}{l}\text { Static } \\
\text { Buried }\end{array}$ & $\begin{array}{l}\text { Flow } \\
\text { Buried }\end{array}$ \\
\hline \multicolumn{7}{|c|}{ Survival after 10-d exposure a } \\
\hline $\begin{array}{l}\text { Sheepshead minnow } \\
\text { Cyprinodon } \\
\text { variegates }\end{array}$ & $100(0)$ & $100(0)$ & $100(0)$ & $100(0)$ & $100(0)$ & 100 \\
\hline $\begin{array}{l}\text { Mussel (adult) } \\
\text { Mytilus } \\
\text { galloprovincialis }\end{array}$ & $100(0)$ & $100(0)$ & $100(0)$ & $100(0)$ & $100(0)$ & 100 \\
\hline $\begin{array}{l}\text { Amphipod } \\
\text { E. estuarius }\end{array}$ & $100(0)$ & $90(10)$ & $90(7.1)$ & $90(10)$ & $90(5)$ & $95(5)$ \\
\hline $\begin{array}{l}\text { Amphipod } \\
\text { L. plumulosus }\end{array}$ & $62(2.9)$ & $80(7.1)$ & $75(21)$ & $63(13)$ & $73(20)$ & 77 (32) \\
\hline $\begin{array}{l}\text { Polychaete } \\
\text { Neanthes } \\
\text { arenaceodentata }\end{array}$ & 87 (11) & $80(0)$ & 70 (14.1) & $80(20)$ & $87(12)$ & $93(12)$ \\
\hline \multicolumn{7}{|c|}{ Development success after 2-d exposure a } \\
\hline $\begin{array}{l}\text { Mussel (embryo) } \\
\text { Mytilus } \\
\text { galloprovincialis }\end{array}$ & $70(16)$ & $74(16)$ & 0 & 85 (13) & $73(22)$ & $85(13)$ \\
\hline
\end{tabular}

a Data is presented as mean ( \pm 1 standard deviation) 
The only test endpoint in which statistically significant effects were observed was for mussel embryo-larval development in which the SE treatment, the treatment representing the worst-case scenario, resulted in no normally developed larvae (Table 7.1). No other treatments resulted in significantly lower larval development success when compared to controls. The impacts to mussel larval development in the SE treatment are explained by relatively high overlying water TNT concentrations ( $\left.0.4 \mathrm{mg} \mathrm{L}^{-1}\right)$ measured on the day that the mussel embryo exposures were initiated, which approached thresholds for TNT exposure in an experiment earlier in this study (median effective concentration $=0.75 \mathrm{mg} \mathrm{L}^{-1}$; Rosen and Lotufo 2007a; see Section 4).

\section{Bioaccumulation of MC released from Composition B}

The bioaccumulation of TNT and RDX from Composition B and their transformation products in fish, mussel, polychaetes and amphipods were measured at the end of the 10-d exposures. Whole body concentrations (body burden) of RDX and SumTNT, for those tissues in which these compounds were detectable, are presented in Table 7.2. Neither TNT nor RDX were detectable in amphipod or polychaete tissues. Bioconcentration values for fish and mussel tissues were similar overall, and corresponded with overlying water concentrations, with SE (worst case) treatments having the highest body burdens.

Table 7.2. Body burdens from Composition B fragment exposures

\begin{tabular}{|c|c|c|c|c|}
\hline \multirow[b]{2}{*}{ Species } & \multirow[b]{2}{*}{ Treatment } & \multicolumn{3}{|c|}{ Body Burdena } \\
\hline & & $\begin{array}{l}\text { RDX } \\
\left(\mathrm{nmol} \mathrm{g}^{-1}\right)\end{array}$ & $\begin{array}{l}\text { SumTNT } \\
(\mathrm{nmol} \mathrm{g-1)})\end{array}$ & $\begin{array}{l}\text { Am-DNTs as \% } \\
\text { of SumTNT }\end{array}$ \\
\hline \multirow{4}{*}{$\begin{array}{l}\text { Sheepshead } \\
\text { minnow } \\
\text { Cyprinodon } \\
\text { variegatus }\end{array}$} & Static Exposed & $35.3(29.9)$ & $28.2(12.6)$ & 89 (6.6) \\
\hline & Static Buried & $0.4(0.7)$ & BDL & BDL \\
\hline & Flow Exposed & $10.8(4.4)$ & $13.7(4.4)$ & $79(4.4)$ \\
\hline & Flow Buried & BDL & BDL & BDL \\
\hline \multirow{4}{*}{$\begin{array}{l}\text { Mussel (adult) } \\
\text { Mytilus } \\
\text { galloprovincialis }\end{array}$} & Static Exposed & $46.4(9.1)$ & $31.1(8.2)$ & $82(8.4)$ \\
\hline & Static Buried & BDL & BDL & BDL \\
\hline & Flow Exposed & $31.5(7.5)$ & $18.7(8.5)$ & $34(5.1)$ \\
\hline & Flow Buried & BDL & BDL & BDL \\
\hline \multirow{3}{*}{$\begin{array}{l}\text { Amphipod } \\
\text { Eohaustorius } \\
\text { estuarius }^{b}\end{array}$} & Static Exposed & 108 & 74.4 & 87 \\
\hline & Static Buried & $\mathrm{BDL}$ & BDL & BDL \\
\hline & Flow Exposed & BDL & BDL & BDL \\
\hline
\end{tabular}

a Data is presented as mean ( \pm 1 standard deviation), ${ }^{b}$ Replicates composited due to insufficient tissue mass. BDL - below detection limit. 
Whole body residues were below levels associated with biological effects determined in previous studies (Section 3). The highest mean body residue in mussels (Table 7.2) was lower than the no-observed-effect residues (NOER) reported for that species (45.3 and $86.2 \mathrm{nmol} \mathrm{g}^{-1}$, for TNT and RDX, respectively) (Rosen and Lotufo 2007a). The highest mean body residues in the sheepshead minnow were $28.2 \mathrm{nmol} \mathrm{g}^{-1}$ sumTNT (the sum of $25.1 \mathrm{nmol} \mathrm{g}^{-1} \mathrm{Am}$-DNTs and $3.1 \mathrm{nmol} \mathrm{g}^{-1} \mathrm{TNT}$ ) and $35.3 \mathrm{nmol} \mathrm{g}^{-1} \mathrm{RDX}$. Those residues are lower by approximately 6 -fold relative to the $5^{-\mathrm{d} \text { median }}$ lethal residue $\left(\mathrm{LR}_{50}\right)$ determined for TNT $\left(20.4 \mathrm{nmol} \mathrm{g}^{-1}\right)$, 2-Am-DNT (275 $\mathrm{nmol} \mathrm{g}^{-1}$ ), and approximately 4-fold relative to the RDX 10-d NOER $\left(135 \mathrm{nmol} \mathrm{g}^{-1}\right.$ ) (Lotufo et al. 2010a, 2010b). Because of the large difference in the toxicity of TNT and Am-DNTs, measurement of the concentration of the TNT and its major transformation products in the exposure water is critical for the appropriate interpretation of body residue data.

\section{Conclusions}

This laboratory experiment simulated real world exposure of marine receptors to Composition $\mathrm{B}$ following a low order detonation, providing insight into more realistic assessment of fate and effects of $\mathrm{MC}$ in underwater environments. A conservative flow rate resulted in substantially reduced TNT and RDX overlying and porewater concentrations (2-3 fold) and lower body residues (about a factor of 2) when compared to static treatments. Burial of Composition B fragments resulted in very limited movement of $\mathrm{MC}$ compounds to overlying water and no detectable tissue concentrations to organisms. Therefore, it is expected that buried munitions in the field would pose a lower risk to organisms inhabiting the water column relative to surface exposed munitions. Porewater TNT and RDX concentrations were highest immediately under the Composition B fragments, yet were dramatically lower a few centimeters from the fragment, suggesting only very localized elevated exposure potential to infaunal organisms residing directly under Composition B fragments. TNT and RDX approached steady concentrations in overlying water and porewater that were much lower than maximum attainable concentrations based on solubility limits of these compounds. Adverse effects to amphipods, adult mussels, fish, and polychaetes under these conservative exposure scenarios (e.g. low or no flow, sandy sediment) were not observed. Mussel embryolarval development was the only endpoint for which effects were observed, and only under static conditions with exposed fragment. As this experiment was designed to represent a worst case scenario, and resulted in little bioaccumulation and toxicity, it is expected that ecological risk from Composition $\mathrm{B}$, and likely other $\mathrm{MC}$, in marine systems is negligible. 


\section{Summary of aquatic toxicology data for select MC}

This section provides an abbreviated summary of the available data on fate toxicity and bioaccumulation of explosive MC in aquatic environments. More detailed information and citations are provided in Sections 2-7 of this report.

The molecular formula for 2,4,6-trinitrotoluene (TNT) is $\mathrm{C}_{7} \mathrm{H}_{5} \mathrm{~N}_{3} \mathrm{O}_{6}$, with a corresponding molecular weight of $227.1 \mathrm{~g} \mathrm{~mol}^{-1}$. At room temperature, TNT is a yellow solid that is slightly soluble in water. The water solubility of TNT is $130 \mathrm{mg} \mathrm{L}^{-1}$ at $25^{\circ} \mathrm{C}$. Dissolved TNT will diffuse and disperse with the water in which it is dissolved.

Photochemical transformation (i.e., photolysis or photooxidation) of TNT in aqueous solutions has been investigated. When associated with organic matter in the water column, soil or sediment, TNT typically transforms, sometimes extensively, to reduced aminated compounds, first to produce aminodinitrotoluene (Am-DNT) and preferentially 4-Am-DNT, with further reduction producing diaminonitrotoluene (DANT) and preferentially 2,4DANT. Strong sorption to sediment organic matter not attributed to hydrophobic partitioning has been reported for both freshwater and marine sediment.

In water, lethal toxicity values, reported for ten marine species, varied widely, from $0.98 \mathrm{mg} \mathrm{L}^{-1}$ ( $\mathrm{LC}_{50}$ for a mysid shrimp) to $19.5 \mathrm{mg} \mathrm{L}^{-1}$ ( $\mathrm{LC}_{50}$ for the adult stage of a mussel). Lethal toxicity to freshwater species has been reported within a similar range. Sublethal toxicity has been reported as impairment of embryo development at $0.75 \mathrm{mg} \mathrm{L}^{-1}$ (LOEC) for a mussel and $12 \mathrm{mg} \mathrm{L}^{-1}$ (reported effect concentration) for a sea urchin, as well as decrease growth of an alga germling at $0.61 \mathrm{mg} \mathrm{L}^{-1}\left(\mathrm{EC}_{50}\right)$. Experiments on the fate and effects of TNT on fish showed no significant impacts on body indices, hematological variables, hepatic detoxification, and antioxidant enzyme activities. 
In sediment, lethal toxicity values, reported for three marine invertebrate species, varied from $38 \mathrm{mg} \mathrm{kg}^{-1}$ (LOEC for an amphipod) to $508 \mathrm{mg} \mathrm{kg}^{-1}$ (LOEC for a polychaete). Lethal toxicity values for freshwater invertebrates, as LOEC, varied widely from $0.1 \mathrm{mg} \mathrm{kg}^{-1}$ to $69 \mathrm{mg} \mathrm{kg}^{-1}$.

Bioconcentration factor values were $0.31 \mathrm{ml} \mathrm{g}^{-1}$ (for a mussel) and $9.71 \mathrm{ml} \mathrm{g}^{-1}$ (for sheepshead minnow), indicating that tissue residues are expected to reach only up to few folds the exposure concentration. For freshwater species, bioconcentration factor values ranged from 0.8 to $5.0 \mathrm{ml} \mathrm{g}^{-1}$. TNT is efficiently transformed to 2-Am-DNT and 4-Am-DNT in the tissue. Parent and transformation products are quickly eliminated. Elimination half lives were 0.33 (mussel) and $0.91 \mathrm{~h}$ (sheepshead minnow), indicating that release from exposure would result in fast depuration and likely recovery from toxic effects.

\section{2-Am-DNT}

The molecular formula for 2-amino-4,6-dinitrotoluene (2-Am-DNT) is $\mathrm{C}_{7} \mathrm{H}_{7} \mathrm{~N}_{3} \mathrm{O}_{4}$ with a corresponding molecular weight of $197.2 \mathrm{~g} \mathrm{~mol}^{-1}$ (USEPA 2012). The water solubility of $2-\mathrm{Am}-\mathrm{DNT}$ is $42 \mathrm{mg} \mathrm{L}^{-1}$ at $25^{\circ} \mathrm{C}$. The presence of 2-Am-DNT in the environment results primarily from the microbial reduction of the 2-nitro group of TNT.

In water, the lethal toxicity values for 2-Am-DNT ( $8.6 \mathrm{mg} \mathrm{L}^{-1}, \mathrm{LC}_{50}$ for sheepshead minnow and a marine copepod (no lethal effects at $50 \mathrm{mg} \mathrm{L}^{-1}$ ) were higher than those for TNT. The toxicity of 2-Am-DNT was lower than that of TNT for freshwater fish and most invertebrate species investigated with the exception being cladocerans. Marine toxicity data for 2-Am-DNT in sediment have not been found. For a freshwater midge, 2-Am-DNT was less toxic (59 $\mathrm{mg} \mathrm{kg}^{-1}$, LOEC) than TNT.

A single bioconcentration factor value ( $13.1 \mathrm{ml} \mathrm{g}^{-1}$ for sheepshead minnow) has been reported. For freshwater species, bioconcentration factor values were $10.2 \mathrm{ml} \mathrm{g}^{-1}$ for 2-Am-DNT and 12.4 for 4-Am-DNT. Elimination halflife was $0.72 \mathrm{~h}$ (sheepshead minnow), indicating that release from exposure would result in fast depuration and likely recovery from toxic effects.

\section{4-Am-DNT}

The molecular formula for 4-amino-2,6-dinitrotoluene (4-Am-DNT) is $\mathrm{C}_{7} \mathrm{H}_{7} \mathrm{~N}_{3} \mathrm{O}_{4}$ with a corresponding molecular weight of $197.2 \mathrm{~g} \mathrm{~mol}^{-1}$. The 
water solubility of $4-\mathrm{Am}-\mathrm{DNT}$ is $42 \mathrm{mg} \mathrm{L}^{-1}$ at $25^{\circ} \mathrm{C}$. No data on the fate, biotransformation or marine toxicity was found for 4-Am-DNT. The presence of 4-Am-DNT in the environment results primarily from the bacterial reduction of the 4-nitro group of TNT.

Lethal toxicity values for freshwater aquatic vertebrates and invertebrates varied widely, from $5.1 \mathrm{mg} \mathrm{L}^{-1}\left(\mathrm{LC}_{50}\right.$ for a cladoceran) to $22.7 \mathrm{mg} \mathrm{L}^{-1}\left(\mathrm{LC}_{50}\right.$ for the larval stage of a frog). Sublethal reproductive effects $\left(\mathrm{EC}_{50}\right)$ on a freshwater cladoceran were noted at $5.1 \mathrm{mg} \mathrm{L}^{-1}$. Freshwater sediment toxicity data for 4-Am-DNT have not been found.

Although marine bioaccumulation data for 4-Am-DNT have not been found, based on the low $\log \mathrm{K}_{\text {ow }}$ the potential for bioconcentration in aquatic organisms is considered low. A single freshwater bioconcentration factor (12.4 $\mathrm{ml} \mathrm{g}^{-1}$ for an oligochaete) has been reported.

\section{2,4-DANT}

The molecular formula for 2,4-diamino-6-nitrotoluene (2,4-DANT) is $\mathrm{C}_{7} \mathrm{H}_{9} \mathrm{~N}_{3} \mathrm{O}_{2}$ with a corresponding molecular weight of $167.2 \mathrm{~g} \mathrm{~mol}^{-1}$. The presence of 2,4-DANT in the environment results primarily from the microbial reduction of the 2 and 4-nitro group of TNT.

In saline water, exposure to 2,4-DANT at $50 \mathrm{mg} \mathrm{L}^{-1}$ failed to promote lethal effects on sheepshead minnows or copepods, suggesting that near complete nitro group reduction may substantially decrease the toxicity of TNT. Conversely, the toxicity of 2,4-DANT was substantially higher (0.05 $\mathrm{mg} \mathrm{L}^{-1}$, LOEC) than that of TNT for the reproduction of a cladoceran. In sediment, lethal toxicity values reported for two invertebrate species, were $3.0 \mathrm{mg} \mathrm{kg}-1$ (LOEC for an amphipod) and $574 \mathrm{mg} \mathrm{kg}^{-1}$ (LOEC for a polychaete). For freshwater invertebrates, sediment-associated 2,4-DANT (0.3 and $59 \mathrm{mg}$ $\mathrm{kg}^{-1}$ LOEC for an amphipod and midge, respectively) and TNT were toxic at similar concentrations. Marine or freshwater toxicity data for 2,4-DANT in sandy sediment have not been found.

A single bioconcentration factor value ( $0.51 \mathrm{ml} \mathrm{g}^{-1}$ for sheepshead minnow) has been reported. A bioconcentration factor of $2.8 \mathrm{ml} \mathrm{g}^{-1}$ has been reported for a freshwater oligochaete. The elimination half life for 2,4-DANT for sheepshead minnow was $0.25 \mathrm{~h}$, indicating that release from exposure would result in fast depuration and likely recovery from toxic effects. 


\section{2,6-DANT}

The molecular formula for 2,6-diamino-4-nitrotoluene (2,6-DANT) is $\mathrm{C}_{7} \mathrm{H}_{9} \mathrm{~N}_{3} \mathrm{O}_{2}$ with a corresponding molecular weight of $167.2 \mathrm{~g} \mathrm{~mol}^{-1}$. The presence of 2,6-DANT in the environment results primarily from the microbial reduction of the 2 and 6-nitro group of TNT. No data on the fate, biotransformation or marine toxicity was found for 2,6-DANT.

In water, exposure to 2,6-DANT at $50 \mathrm{mg} \mathrm{L}^{-1}$ failed to promote lethal effects on marine copepods (Won et al. 1976). The toxicity of 2,6-DANT was substantially lower (0.33 $\mathrm{mg} \mathrm{L}^{-1}$, LOEC) than that of TNT for the reproduction of a cladoceran. No sediment toxicity data for 2,6-DANT were found.

In sediment, lethal toxicity values, reported for two invertebrate species, were $3.0 \mathrm{mg} \mathrm{kg}^{-1}$ (LOEC for an amphipod) and $574 \mathrm{mg} \mathrm{kg}^{-1}$ (LOEC for a polychaete). For freshwater invertebrates, 2,4-DANT (0.3 and $59 \mathrm{mg} \mathrm{kg}^{-1}$ LOEC for an amphipodand midge, respectively) and TNT were toxic at similar concentrations. Marine or freshwater toxicity data for 2,4-DANT in sandy sediment have not been found.

A single bioconcentration factor value ( $0.51 \mathrm{ml} \mathrm{g}^{-1}$ for sheepshead minnow) has been reported. A bioconcentration factor of $2.8 \mathrm{ml} \mathrm{g}^{-1}$ has been reported for a freshwater oligochaete. The elimination half life for 2,4-DANT for sheepshead minnow was $0.25 \mathrm{~h}$, indicating that release from exposure would result in fast depuration and likely recovery from toxic effects.

\section{1,3,5-TNB}

The molecular formula for 1,3,5-trinitrobenzene (1,3,5-TNB) is $\mathrm{C}_{6} \mathrm{H}_{3} \mathrm{~N}_{3} \mathrm{O}_{6}$, with a corresponding molecular weight of $213.1 \mathrm{~g} \mathrm{~mol}^{-1}$ (USEPA 2012). At room temperature, 1,3,5-TNB is a clear to light yellow solid. The water solubility of $1,3,5$-TNB is $385 \mathrm{mg} \mathrm{L}^{-1}$ at $25^{\circ} \mathrm{C} .1,3,5$-TNB is also formed as a by-product of TNT manufacture and as a product of TNT environmental transformation, including its photolysis. Extensive transformation of 1,3,5TNB has been shown to occur in freshwater and marine sediment. Reductive transformation of $1,3,5$-TNB, leading to the formation of dinitroaniles (or amino-dinitrobenzenes) has been reported. In marine and freshwater fine-grained sediment, extensive transformation of parent compound to aminated products has been reported. 
In water, lethal toxicity values, reported for four marine species, varied narrowly from $0.74 \mathrm{mg} \mathrm{L}^{-1}\left(\mathrm{LC}_{50}\right.$ for a mysid shrimp) to $1.6 \mathrm{mg} \mathrm{L}^{-1}\left(\mathrm{LC}_{50}\right.$ for a polychaete). Lethal toxicity to freshwater species has been reported within a similar range $\left(1.0-2.7 \mathrm{mg} \mathrm{L}^{-1}\right)$. Sublethal toxicity to marine invertebrates has been reported as impairment of sea urchin embryo development at $1.3 \mathrm{mg} \mathrm{L}^{-1}$ (LOEC) and impaired reproduction for a polychaete $\left(0.4 \mathrm{mg} \mathrm{L}^{-1}, \mathrm{EC}_{50}\right)$.

In sediment, lethal toxicity values, reported for two marine invertebrate species, were $13 \mathrm{mg} \mathrm{kg}^{-1}$ (LOEC for an amphipod) and $107 \mathrm{mg} \mathrm{kg}^{-1}$ (highest concentration tested, no significant effect for a polychaete). The lowest lethal toxicity value for a freshwater invertebrate was $1 \mathrm{mg} \mathrm{kg}^{-1}$ (LOEC for an amphipod). Although no data for the bioaccumulation of $1,3,5$-TNB in marine or freshwater fish and invertebrates were found, based on the low $\log \mathrm{K}_{\text {ow }}$, the potential for bioconcentration in aquatic organisms is considered low.

\section{2,4-DNT}

The molecular formula for 2,4-dinitrotoluene (2,4-DNT) is $\mathrm{C}_{7} \mathrm{H}_{6} \mathrm{~N}_{2} \mathrm{O}_{4}$ with a corresponding molecular weight of $182.2 \mathrm{~g} \mathrm{~mol}^{-1}$. At room temperature, 2,4DNT is a yellow crystalline solid. The water solubility of 2,4 -DNT is $270 \mathrm{mg}$ $\mathrm{L}^{-1}$ at $25^{\circ} \mathrm{C}$. Dissolved 2,4-DNT will diffuse and disperse with the water it is dissolved in.

DNTs are a primary product in propellants and are also formed as a byproduct during the TNT manufacturing process. There are six isomers of DNT, the most common one is 2,4-DTN. Technical grade DNT is composed of approximately $75 \% 2,4$-DNT, $20 \% 2,6$-DNT, and $5 \%$ other isomers. Like TNT, DNTs can be reduced to the corresponding mono or diamino derivatives. Strong sorption of 2,4-DNT to soil organic matter has been reported. There are six isomers of DNT, the most common one is 2,4-DNT.

In water, lethal toxicity values, reported for four marine species, varied widely, from $4.4 \mathrm{mg} \mathrm{L}^{-1}\left(\mathrm{LC}_{50}\right.$ for a mysid shrimp) to $48 \mathrm{mg} \mathrm{L}^{-1}\left(\mathrm{LC}_{50}\right.$ for redfish). Sublethal toxicity to marine invertebrates has been reported as impaired sea urchin embryo development at $10 \mathrm{mg} \mathrm{L}^{-1}$ (LOEC) and impaired polychaete reproduction $\left(5.2 \mathrm{mg} \mathrm{L}^{-1}, \mathrm{EC}_{50}\right)$. No toxicity data for 2,4 -DNT in marine or freshwater sediment were found. 
Although no data for the bioaccumulation of 2,4-DNT in marine fish and invertebrates were found, based on the low $\log \mathrm{K}_{\mathrm{ow}}$ the potential for bioconcentration in aquatic organisms is considered low. For freshwater species, reported bioconcentration factor values were $4.5 \mathrm{ml} \mathrm{g}^{-1}$ for gold fish and $9.2 \mathrm{ml} \mathrm{g}^{-1}$ for carp.

\section{2,6-DNT}

The molecular formula for 2,6-dinitrotoluene (2,6-DNT) is $\mathrm{C}_{7} \mathrm{H}_{6} \mathrm{~N}_{2} \mathrm{O}_{4}$ with a corresponding molecular weight of $182.2 \mathrm{~g} \mathrm{~mol}^{-1}$. At room temperature, 2,6DNT is a tan crystalline solid. The water solubility of 2,6-DNT is $206 \mathrm{mg} \mathrm{L}^{-1}$ at $25^{\circ} \mathrm{C}$. Dissolved 2,6-DNT will diffuse and disperse with the water it is dissolved in.

In saline water, lethal toxicity values, reported for three marine species, varied widely, from $5.0 \mathrm{mg} \mathrm{L}^{-1}\left(\mathrm{LC}_{50}\right.$ for a mysid shrimp) to $28 \mathrm{mg} \mathrm{L}^{-1}\left(\mathrm{LC}_{50}\right.$ for redfish). Lowest sublethal toxicity values to marine invertebrates were reported as impaired sea urchin embryo development at $6.7 \mathrm{mg} \mathrm{L}^{-1}$ (LOEC) and impaired reproduction ( $2.1 \mathrm{mg} \mathrm{L}^{-1}, \mathrm{EC}_{50}$ for a polychaete). In marine sediment, no significant lethal effects to an amphipod were observed at the highest 2,6-DNT measured tested concentration (5 $\left.\mathrm{mg} \mathrm{kg}^{-1}\right)$.

Although no data for the bioaccumulation of 2,6-DNT in marine fish and invertebrates has been found, the potential for bioconcentration in aquatic organisms is considered low based on its low log $\mathrm{K}_{\text {ow. }}$. For freshwater species, the sole reported bioconcentration factor value was $1.7 \mathrm{ml} \mathrm{g}^{-1}$ for carp.

\section{Tetryl}

The molecular formula for tetryl (methyl-2,4,6-trinitrophenylnitramine) is $\mathrm{C}_{7} \mathrm{H}_{5} \mathrm{~N}_{5} \mathrm{O}_{8}$ with a corresponding molecular weight of $287.2 \mathrm{~g} \mathrm{~mol}^{-1}$. At room temperature, tetryl is a yellow, crystalline solid. The water solubility of tetryl is $75 \mathrm{mg} \mathrm{L}^{-1}$ at $25^{\circ} \mathrm{C}$. Dissolved tetryl will diffuse and disperse with the water it is dissolved in.

Photolysis and hydrolysis are major environmental transformation processes for tetryl in aqueous media. In natural, sunlit waters, tetryl is transformed via photolysis and hydrolysis to several degradation products, including picric acid, which is the primary hydrolysis product of tetryl. Information on the fate of tetryl in sediments is limited, and it is reported 
that the formation of unknown breakdown products occurs in sandy and fine-grained marine sediments spiked with tetryl. Picric acid, a primary hydrolysis product of tetryl, was detected in sandy sediment only.

In saline water, lethal toxicity values, reported for three marine species, varied widely, from $0.03 \mathrm{mg} \mathrm{L}^{-1}\left(\mathrm{LC}_{50}\right.$ for a polychaete) to $1.1 \mathrm{mg} \mathrm{L}^{-1}\left(\mathrm{LC}_{50}\right.$ for redfish). Sublethal toxicity to marine invertebrates has been reported as impaired polychaete reproduction ( $0.01 \mathrm{mg} \mathrm{L}^{-1}, \mathrm{EC}_{50}$ ), impaired sea urchin embryo development ( $0.05 \mathrm{mg} \mathrm{L}^{-1}, \mathrm{EC}_{50}$ ), and decrease growth of an alga germling at $0.20 \mathrm{mg} \mathrm{L}^{-1}\left(\mathrm{EC}_{50}\right)$. In sediment, the sole lethal toxicity value reported was $4.0 \mathrm{mg} \mathrm{kg}^{-1}$ (LOEC for sandy sediment) for an amphipod species (Table 5.3). Although no data for the bioaccumulation of tetryl in marine or freshwater fish and invertebrates were found, based on the low $\log \mathrm{K}_{\mathrm{ow}}$, the potential for bioconcentration in aquatic organisms is considered low.

\section{Explosive D (Ammonium Picrate)/Picric Acid}

The molecular formula for ammonium picrate (2,4,6-trinitrophenol ammonium salt), which is also known as Explosive D, is $\mathrm{C}_{6} \mathrm{H}_{3} \mathrm{~N}_{3} \mathrm{O}_{7} \cdot \mathrm{H}_{3} \mathrm{~N}$ with a corresponding molecular weight of $246.1 \mathrm{~g} \mathrm{~mol}^{-1}$. Ammonium picrate is a salt formed from picric acid and ammonium cation. The molecular formula for picric acid is $\mathrm{C}_{6} \mathrm{H}_{3} \mathrm{~N}_{3} \mathrm{O}_{7}$ with a corresponding molecular weight of $229.1 \mathrm{~g} \mathrm{~mol}^{-1}$. At room temperature, ammonium picrate and picric acid are bright yellow, crystalline solids. The water solubility of ammonium picrate is $10,200 \mathrm{mg} \mathrm{L}^{-1}$ and for picric acid is $12,800 \mathrm{mg} \mathrm{L}^{-1}$, both at $25^{\circ} \mathrm{C}$. Ammonium picrate is an ammonium salt of picric acid and under normal aqueous conditions will dissociate to ammonium and picrate ions and will reside in the water column as picric acid.

In sediment slurry tests, picric acid was found to remain in the aqueous phase with little partitioning to the sediments. Transformation to picramic acid was limited. It is likely that the ions transform further depending on the oxidation-reduction potential.

Similar to TNT, picric acid is also degraded through reduction, and the microbial biodegradation of picric acid results in the formation of 2A-dinitrophenol. Under aerobic conditions, the mono- or dinitrophenol can then be oxygenated with subsequent ring cleavage. Investigation of the fate of picric acid in marine sediments revealed that transformation rates were highest in incubated, fine-grained sediment and major transforma- 
tion products included 4-dinitrophenol, aminodinitrophenols (including picramic acid), 3,4-diaminophenol, amino nitrophenol, and nitrodiaminophenol. Picric acid has been reported to bind to sediment and resist hydrolysis, biodegradation and photolysis.

In water, lethal toxicity values for picric acid, reported for seven marine species, varied widely, from $13 \mathrm{mg} \mathrm{L}^{-1}\left(\mathrm{LC}_{50}\right.$ for a mysid shrimp) to $265 \mathrm{mg}$ $\mathrm{L}^{-1}\left(\mathrm{LC}_{50}\right.$ for a polychaete). Sublethal toxicity as impaired reproduction has been reported as $60 \mathrm{mg} \mathrm{L}^{-1}$ for a copepod and $155 \mathrm{mg} \mathrm{L}^{-1}$ for a polychaete ( $\mathrm{EC}_{50}$ values), $28 \mathrm{mg} \mathrm{L}^{-1}$ for impairment of oyster shell deposition $\left(\mathrm{EC}_{50}\right)$, $281 \mathrm{mg} \mathrm{L}^{-1}$ for impairment of sea urchin embryo development $\left(\mathrm{EC}_{50}\right)$, and decrease growth of an alga germling at $94 \mathrm{mg} \mathrm{L}^{-1}\left(\mathrm{EC}_{50}\right)$. The breakdown products picramic acid and 2,4-DNP have been reported as more toxic than the parent compound for marine species, typically by about an order of magnitude. In freshwater species, there was about an order of magnitude difference in toxicity for trout and a cladoceran but three orders of magnitude greater toxicity for 2,4-DNP to the bluegill.

In marine sediment, the sole lethal toxicity value was $162 \mathrm{mg} \mathrm{kg}^{-1}$ (LOEC for sandy sediment) for an amphipod species. In fine-grained sediment, amphipod mortality was highest at an intermediate concentration in which picric acid was below the detection limit, with the toxicity attributed to unknown transformation products. Although no data for the bioaccumulation of picric acid in marine or freshwater fish and invertebrates were found, based on the low $\log \mathrm{K}_{\mathrm{ow}}$ the potential for bioconcentration in aquatic organisms is considered low.

\section{RDX}

Royal Demolition Explosive, or RDX (cyclo-1,3,5-trimethylene-2,4,6trinitramine), has the molecular formula $\mathrm{C}_{3} \mathrm{~N}_{6} \mathrm{O}_{6} \mathrm{H}_{6}$ and has a molecular weight of $222.26 \mathrm{~g} / \mathrm{mol}$. At room temperature, RDX is a colorless crystal. The water solubility of RDX is $56.3 \mathrm{mg} \mathrm{L}^{-1}$ at $25^{\circ} \mathrm{C}$. Military grade RDX generally contain up to $10 \% \mathrm{HMX}$ as an impurity of the manufacturing process.

The $\log \mathrm{K}_{\mathrm{oc}}$ is 2.00 and indicates that moderate partitioning into organic carbon will occur. However, the $\log \mathrm{K}_{\mathrm{ow}}$ value is very low such that almost no partitioning into a non-polar substance will occur. With all physical factors of RDX taken into account, it is a compound that will reside on the 
water body floor as a solid with dissolution into the water column over time.

The biodegradation of RDX occurs under both aerobic and anaerobic conditions but the anaerobic process is significantly faster. Unlike nitroaromatic explosives, RDX undergoes ring cleavage and mineralization under both aerobic and anaerobic conditions. Aerobically, it has been found that microbial cleavage of one of the $\mathrm{N}-\mathrm{NO}_{2}$ bonds produces unstable intermediates. Subsequent degradation reactions are largely governed by the interactions of the newly formed intermediates with water and eventually produce $\mathrm{NO}^{-}, \mathrm{N}_{2} \mathrm{O}, \mathrm{NH}_{3}, \mathrm{HCHO}$, and $\mathrm{HCOOH}$. Anaerobically, sequential reduction of the nitro groups to produce mono-, di- and trinitroso derivatives (MNX, DNX and TNX, respectively) is most frequently observed. The nitroso derivatives may be further transformed to produce the unstable hydroxylamine derivatives, leading to ring cleavage.

A study of microbial degradation of RDX in tropical marine sediments collected in Hawaii indicated that cyclic nitramine contaminants are likely to be degraded upon release from munitions into tropical marine sediment, suggesting natural attenuation in situ. Extensive transformation of RDX occurred following spiking to fine-grained sediment, but the transformation products were not identified. Different from the fate of RDX in organicallyrich sediment, transformation was minimal in sandy sediment.

In water, lethal toxicity was evaluated for sixteen marine species. For most species, no significant lethality has occurred at concentrations reported as at or near the solubility limit. Effects concentration ranged from $2.4 \mathrm{mg} \mathrm{L}^{-1}$ ( $\mathrm{LC}_{50}$ for speckled sand dab) to $26 \mathrm{mg} \mathrm{L}^{-1}\left(\mathrm{EC}_{50}\right.$ for reproduction impairment of a polychaete). Lethal toxicity to freshwater species has been reported within similar range.

In sediment, no significant lethality has been reported for marine or freshwater species in sandy and fine-grained sediments, with no effects concentrations ranging from 102 to $2229 \mathrm{mg} \mathrm{kg}^{-1}$.

Bioconcentration factor values were $0.7 \mathrm{ml} \mathrm{g}^{-1}$ (for a mussel) and $1.7 \mathrm{ml} \mathrm{g}^{-1}$ (for sheepshead minnow), indicating that tissue residues are expected to only slightly exceed the exposure concentration. For freshwater species, bioconcentration factor values ranged from 1.0 to $2.1 \mathrm{ml} \mathrm{g}^{-1}$. No reports of RDX biotransformation in fish or invertebrates were found. Elimination 
half lives were 0.27 (mussel) and $7.7 \mathrm{~h}$ (sheepshead minnow), indicating that release from exposure would result in fast depuration and likely recovery from toxic effects.

\section{HMX}

Octahydro-1,3,5,7-tetranitro-1,3,5,7-tetrazocine, also known as highmelting explosive (HMX), is a colorless polycrystalline solid with a molecular formula of $\mathrm{C}_{4} \mathrm{H}_{8} \mathrm{~N}_{8} \mathrm{O}_{8}$ and a molecular weight of $296.20 \mathrm{~g} / \mathrm{mol}$. The water solubility of HMX is $4.5 \mathrm{mg} \mathrm{L}^{-1}$ at $25^{\circ} \mathrm{C}$. Military grade RDX generally contains up to $10 \% \mathrm{HMX}$ as an impurity of the manufacturing process (Talmage et al. 1999).

HMX exists in four polymorphic forms: $\alpha, \beta, \gamma$, and $\delta$. The $\beta$ form of HMX is the least sensitive to impact, and the most preferred for use as a high explosive. The $\log \mathrm{K}_{\mathrm{ow}}$ value for HMX is very low such that almost no partitioning into a non-polar substance will occur. With all physical factors of HMX taken into account, it is a compound that will reside on the water body floor as a solid with dissolution into the water column over time.

In water, lethal toxicity was evaluated for only two marine species. Concentrations reported as at or near the solubility limit have not caused significant lethality or sublethal effects. In freshwater, toxicity was evaluated for eight species; only fathead minnows at $7 \mathrm{~d}$ post hatch were lethally impacted at the target concentration of $15 \mathrm{mg} \mathrm{L}^{-1}$, which is higher than the reported solubility limit.

In sediment, no significant lethality was reported for marine or freshwater species in fine-grained sediments, with no effects concentrations ranging from 115 to $353 \mathrm{mg} \mathrm{kg}^{-1}$.

Bioconcentration factor values were $0.31 \mathrm{ml} \mathrm{g}^{-1}$ (for a mussel) and $0.51 \mathrm{ml} \mathrm{g}^{-1}$ (for sheepshead minnow), indicating that tissue residues are expected to be lower than the environmental concentration. No reports of HMX biotransformation in fish or invertebrates were found. Elimination halflives were 1.5 (mussel) and $5.8 \mathrm{~h}$ (sheepshead minnow), indicating that release from exposure would result in fast depuration and likely recovery from toxic effects. 


\section{Nitroguanidine}

Nitoguanidine is also known by its military abrievation: NQ. The molecular formula for nitroguanidine is $\mathrm{CH}_{4} \mathrm{~N}_{4} \mathrm{O}_{2}$ with a corresponding molecular weight of $104.1 \mathrm{~g} \mathrm{~mol}^{-1}$. At room temperature, nitroguanidine is a colorless, crystalline solid. The water solubility of nitroguanidine is $4,400 \mathrm{mg} \mathrm{L}^{-1}$ at $25^{\circ} \mathrm{C}$. Degradation of nitroguanidine was shown to be negligible in activated sludge under aerobic conditions and in sterile sludge under reducing conditions.

In water, no lethal effect to a marine copepod was reported at the highest concentration tested (683 $\left.\mathrm{mg} \mathrm{L}^{-1}\right)$. Similarly, exposure of freshwater fish and aquatic invertebrates to exceedingly high concentrations (1,024 to $2,714 \mathrm{mg}$ $\left.\mathrm{L}^{-1}\right)$ of nitroguanidine also failed to result in toxicity. No sediment toxicity data for nitroguanidine were found. Although no data for the bioaccumulation of nitroguanidine in marine or freshwater fish and invertebrates were found, based on the low $\log \mathrm{K}_{\mathrm{ow}}$, the potential for bioconcentration in aquatic organisms is considered low.

\section{Nitroglycerin}

Nitroglycerin or glycerol trinitrate (propane-1,2,3-triyl trinitrate) is also known by its military abrievation: NG. Nitroglycerin has the molecular formula $\mathrm{C}_{3} \mathrm{H}_{5} \mathrm{~N}_{3} \mathrm{O}_{9}$ with a corresponding molecular weight of $227.1 \mathrm{~g} \mathrm{~mol}^{-1}$. The water solubility of nitroglycerin is $1,800 \mathrm{mg} \mathrm{L}^{-1}$ at $25^{\circ} \mathrm{C}$. At room temperature, nitroglycerin is a colorless liquid. The predominant products of nitroglycerine via hydrolysis are calcium nitrate and calcium nitrite. Nitroglycerine has a low $\log \mathrm{K}_{\mathrm{ow}}$ value, suggesting hydrophilic behavior and a low $\log \mathrm{K}_{\mathrm{oc}}$ value, indicating limited sorption. Nitroglycerine has been documented to transform via microbial mediation in both aerobic and anaerobic conditions, ultimately forming glycerol.

No marine toxicity data for nitroglycerin were found. Lethal toxicity values for freshwater aquatic vertebrates and invertebrates varied widely, from $0.7 \mathrm{mg} \mathrm{L}^{-1}$ ( $\mathrm{LC}_{50}$ for rainbow trout) to $16 \mathrm{mg} \mathrm{L}^{-1}$ (LOEC for a cladoceran). No freshwater sediment toxicity data for nitroglycerin were found. Although no data for the bioaccumulation of nitroglycerin in marine or freshwater fish and invertebrates were found, based on the low $\log \mathrm{K}_{\mathrm{ow}}$ the potential for bioconcentration in aquatic organisms is considered low. 


\section{Nitrocellulose}

Nitrocellulose is also known by its military abrievation: NC. Nitrocellulose is a nitrate ester produced with varying levels of nitration. Thus, the molecular formula is variable and the molecular weight ranges from $10^{5}$ to $10^{6} \mathrm{~g} \mathrm{~mol}^{-1}$. Nitrocellulose is insoluble in water. At room temperature, nitrocellulose is a filamentous yellowish-white particulate. Nitrocellulose is very recalcitrant to microbial degradation. No fate and biotransformation data for nitrocelullose were found.

No marine toxicity data for nitrocelullose were found. Studies with nitrocellulose indicated no toxicity at concentrations up to $1000 \mathrm{mg} \mathrm{L}^{-1}$ when tested with several species of freshwater fish and invertebrates. The overall lack of toxicity of nitrocellulose is likely a result of its insolubility in water. No freshwater sediment toxicity data for nitrocelullose were found. No data for the bioaccumulation of nitrocelullose in marine or freshwater fish and invertebrates were found.

\section{PETN}

PETN, or (pentaerythritol tetranitrate) is used in some booster charges, demolition explosives, and initiating explosives. PETN has the molecular formula $\mathrm{C}_{5} \mathrm{H}_{8} \mathrm{~N}_{4} \mathrm{O}_{12}$ and has a molecular weight of $316.2 \mathrm{~g} / \mathrm{mol}$. At room temperature, PETN is a white, crystalline solid. The water solubility of PETN is uncertain with a reported range of 2.1 to $43 \mathrm{mg} \mathrm{L}^{-1}$ at $25^{\circ} \mathrm{C}$. PETN is not prone to hydrolyze at ambient temperature and is recalcitrant to microbial degradation.

In water, no lethal effect to a marine copepod (the only marine organism tested) occurred at the highest concentration tested (32 $\left.\mathrm{mg} \mathrm{L}^{-1}\right)$. Similarly, exposure of a freshwater invertebrate to $49 \mathrm{mg} \mathrm{L}^{-1}$ (target concentration) also failed to produce a lethal effect, but immobilization was reported after $48 \mathrm{~h}$. No sediment toxicity data for PETN were found. Although no data for the bioaccumulation of PETN in marine or freshwater fish and invertebrates were found, based on the low $\log \mathrm{K}_{\mathrm{ow}}$ the potential for bioconcentration in aquatic organisms is considered low.

\section{DEGDN}

Diethylene glycol dinitrate (DEGDN) is a colorless, odorless, viscous, oily liquid. DEGDN has the molecular formula $\mathrm{C}_{4} \mathrm{H}_{8} \mathrm{~N}_{2} \mathrm{O}_{7}$ and has a molecular 
weight of $196.1 \mathrm{~g} / \mathrm{mol}$. The water solubility of DEGDN is $4,000 \mathrm{mg} \mathrm{L}^{-1}$ at $25^{\circ} \mathrm{C}$. Environmental fate studies indicate that DEGDN is a stable compound once dissolved in water.

No marine toxicity data for DEGDN were found. Lethal toxicity values for freshwater aquatic vertebrates and invertebrates varied from $90.1 \mathrm{mg} \mathrm{L}^{-1}$ ( $\mathrm{LC}_{50}$ for a cladoceran) to $491 \mathrm{mg} \mathrm{L}^{-1}\left(\mathrm{LC}_{50}\right.$ for fathead minnow). No freshwater sediment toxicity data for DEGDN were found. Although no data for the bioaccumulation of DEGDN in marine or freshwater fish and invertebrates were found, based on the low $\log \mathrm{K}_{\mathrm{ow}}$, the potential for bioconcentration in aquatic organisms is considered low. 


\section{References}

Arnot, J. A. 2009. Mass balance models for chemical fate, bioaccumulation, exposure and risk assessment. In V. Simeonov \& M. A. Hassanein (Eds.), Exposure and Risk Assessment of Chemical Pollution - Contemporary Methodology: 69-91.

Arnot, J. A., and FAPC Gobas. 2003. A generic QSAR for assessing the bioaccumulation potential of organic chemicals in aquatic food webs. QSAR and Combinatorial Science 22: 337-345.

Arnot, J. A., and FAPC Gobas. 2006. A review of bioconcentration factor (BCF) and bioaccumulation factor (BAF) assessments for organic chemicals in aquatic organisms. Environmental Reviews. 14:257-297.

Badgujar, D. M., M. B. Talawar, S.N. Asthana, and P.P. Mahulikar. 2008. Advances in science and technology of modern energetic materials: An overview. $J$ Hazard Mater 151: 289-305

Bailey, H. C., and R. J. Spanggord. 1983. The relationship between the toxicity and structure of nitroaromatic chemicals. In: Bishop, W.E., Cardwell, R.D., Heidolph, B.B. (eds.), Aquatic Toxicology and Hazard Assessment: Sixth Symposium, ASTM STP 802. American Society for Testing Materials, Philadelphia, pp. 98-107.

Bailey, H. C., R. J. Spanggord, H. S. Javitz, and D. H. Liu. 1985. Toxicity of TNT Wastewaters to Aquatic Organisms. Final Report. Vol. III. Chronic Toxicity of LAP Wastewater and 2,4,6-Trinitrotoluene. AD-A164 282. Menlo Park, CA: SRI International.

Bakhtiar, R., K. H. Leung, R. A. Stearns, and C. E. Hop. 1997. Evidence for a novel heme adduct generated by the in vitro reaction of 2,4,6-trinitrotoluene with human hemoglobin using electrospray ionization mass spectrometry. J. Inorg. Biochem., 68:273-278.

Belden, J. B., G. R. Lotufo, M. J. Lydy. 2005a. Accumulation of hexahydro-1,3,5-trinitro1,3,5-triazine in channel catfish (Ictalurus punctatus) and aquatic oligochaetes (Lumbriculus variegatus). Environ Toxicol Chem 24:1962-1967.

Belden, J. B., D. R. Ownby, G. R. Lotufo, M. J. Lydy. 2005b. Accumulation of trinitrotoluene (TNT) in aquatic organisms: Part 2 - bioconcentration in aquatic invertebrates and potential for trophic transfer to channel catfish (Ictalurus punctatus). Chemosphere 58:1161-1168.

Belden, J. B., G. R. Lotufo, C. K. Chambliss, J. C. Fisher, D. R. Johnson, R. E. Boyd, J. G. Sims. 2011. Accumulation of ${ }^{14} \mathrm{C}$-trinitrotoluene and related nonextractable (bound) residues in Eisenia fetida. Environ Pollut 159:1368.

Bentley, R. E., J. W. Dean, S. J. Ellis, T. A. Hollister, G. A. LeBlanc, S. Sauter, B. H. Sleight. 1977a. Laboratory evaluation of the toxicity of nitrocellulose to aquatic organisms. Washington, DC: Army Medical Research Development Command. 
Bentley, R. E., J. W. Dean, S. J. Ellis, T. A. Hollister, G. A. LeBlanc, S. Sauter, and B. H. Sleight. 1977b. Laboratory evaluation of the toxicity of cyclotrimethylene trinitramine (RDX) to aquatic organisms. AD A061730. Final Report. EG\&G Bionomics, Wareham, MA for U.S. Army Medical Bioengineering Research and Development Laboratory, Fort Detrick, MD.

Bentley, R. E., G. A. LeBlanc, T. A. Hollister, and B. H. Sleight, III. 1977c. Acute toxicity of 1,3,5,7-tetranitro-octahydro-1,3,5,7-tetrazocine (HMX) to aquatic organisms. Final report. AD Ao61 730. Wareham, MA: EG\&G Bionomics.

Bentley, R. E., J. W. Dean, S. J. Ellis, G. A. LeBlanc, S. Sauter, K. S. Buxter, and B. H. Sleight. 1977d. Laboratory evaluation of the toxicity of nitroglycerine to aquatic organisms. Technical Report, AD A061739. Alexandria, VA: Defense Technical Information Center.

Bentley, R. E., S. R. Petrocelli, and D. C. Suprenant. 1984. Determination of the toxicity to aquatic organisms of HMX and related wastewater constituents. Part III. Toxicity of HMX, TAX and SEX to aquatic organisms. Final report. AD AI72 385. Wareham, MD: Springborn Bionomics, Inc.

Borga, K., K. A. Kidd, D. C. Mui, O. Berglund, J. M. Conder, F. A. Gobas, J. Kucklick, O. Malm, and D. E. Powell. 2012. Trophic magnification factors: Considerations of ecology, ecosystems, and study design. Integrated Environmental Assessment and Management 8: 64-84

Broderius, S. J., M. D. Kahl, G. E. Elonen, D. E. Hammermeister, and M. D. Hoglund. 2005. A comparison of the lethal and sublethal toxicity of organic chemical mixtures to the fathead minnow (Pimephates promelas). Environ Toxicol Chem 24:3117-3127.

Buccafusco, R. J., S. J. Ells and G. A. Leblanc. 1981. Acute toxicity of priority pollutants to bluegill (Lepomis macrochirus). Bulletin of Environmental Contamination and Toxicology 26: 446-452.

Burton, D. T., S. D. Turley, G. T. Peters. 1993. Toxicity of Nitroguanidine, Nitroglycerin, Hexahydro-1,3,5-trinitro-1,3,5-triazine (RDX), and 2,4,6-Trinitrotoluene (TNT) to Selected Freshwater Aquatic Organisms. WREC 93-B3. Queenstown, MD: The University of Maryland System, Agricultural Experiment Station, Wye Research and Education Center.

Burton, D. T., S. D. Turley, G. T. Peters. 1994. The acute and chronic toxicity of hexahydro-1,3,5-trinitro-1,3,5-triazine (RDX) to the fathead minnow (Pimephales promelas). Chemosphere 29:567-579.

Carr, R. S., M. Nipper. 2000. Toxicity of Marine Sediments and Pore Waters Spiked with Ordnance Compounds. Contract Report CR 01-001-ENV. Port Hueneme CA: Naval Facilities Engineering Service Center.

Clausen, J. L. 2011. Energetic residue observations for operational ranges. In Chappell MA, Price CL, George RD, editors. Environmental Chemistry of Explosives and Propellant Compounds in Soils and Marine Systems: Distributed Source Characterization and Remedial Technologies. ACS Symposium Series. American Chemical Society. 
Clausen, J. L., N. Korte. 2011. Fate and transport of energetics from surface soils to groundwater. In Chappell MA, Price CL, George RD, editors. Environmental Chemistry of Explosives and Propellant Compounds in Soils and Marine Systems: Distributed Source Characterization and Remedial Technologies. ACS Symposium Series. American Chemical Society.

Conder, J. M., T. W La Point, J. A. Steevens, G. R. Lotufo. 2004a. Recommendations for the assessment of TNT toxicity in sediment. Environ Toxicol Chem 23:141-149.

Conder, J. M., T. W. La Point, and A. T. Bowen, 2004b. Preliminary kinetics and metabolism of 2,4,6-trinitrotoluene and its reduced metabolites in an aquatic oligochaete. Aquatic Toxicology 69: 199-213.

Conder, J. M., G. R. Lotufo, P. K. Turner, T. W. La Point, J. A. Steevens. 2004c. Solid phase microextraction fibers for estimating the toxicity and bioavailability of sediment-associated organic compounds. Aquatic Ecosystem Health and Management 7:387-397.

Crocker, F., K. Indest, and H. Fredrickson. 2006. Brodegradation of the cyclic nitramine explosives RDX, HMX, and CL-20. Applied Microbiology and Biotechnology 73:274-290.

Cusson, B., A. Latreille, S. Thiboutot, G. Ampleman. 2003. A Great Challenge: Characterization of Sediments from the Lake St. Pierre Firing Range, St. Lawrence River, Quebec, Canada. , 2nd International Symposium on Contaminated Sediments: Characterization, Evaluation, Mitigation/Restoration, Management Strategy Performance, Québec City, Canada.

Darrach, M. R., A. Chutjian, G. A. Plett. 1998. Trace explosives signatures from World War II unexploded undersea ordnance. Environ Sci Technol 32:1354-1358.

Dave, G., E. Nilsson, A-S Wernersson. 2000. Sediment and water phase toxicity and UVactivation of six chemicals used in military explosives. Aquat Ecosyst Health Manage 3:291-299.

Dave, G. 2003. Field test of ammunition (TNT) dumping in the ocean. In Munawar, M, ed, Quality Assessment and Management: Insight and Progress. Aquatic Ecosystem Health \& Management Society.

Davenport, R., L. R. Johnson, D. J. Schaeffer, H. Balbach. 1994. Phototoxicology. 1. Lightenhanced toxicity of TNT and some related compounds to Daphnia magna and Lytechinus variagatus embryos. Ecotoxicol Environ Saf 27:14-22.

Davis, A. D. 2009. Commentary: Munitions Discarded at Sea. Marine Technology Society Journal 43(4): 11-13.

Della Torre, C., F. Corsi, A. Arukwe, L. Alcaro, E. Amato, S. Focardi. 2008a. Effects of 2,4,6-trinitrotoluene (TNT) on phase I and phase II biotransformation enzymes in European eel Anguilla anguilla (Linnaeus, 1758). Mar Environ Res 66:9-11.

Della Torre, C., I. Corsi, S. Focardi, A. Arukwe. 2008b. Effects of 2,4,6-trinitrotoluene (TNT) on neurosteroidogenesis in the European eel (Anguilla anguilla; Linnaeus 1758). Chem Ecol 24:1-7. 
Della Torre, C., I. Corsi, A. Arukwe, M. Valoti, S. Focardi. 2008c. Interactions of 2,4,6trinitrotoluene (TNT) with xenobiotic biotransformation system in European eel Anguilla anguilla (Linnaeus, 1758). Ecotoxicol Environ Saf 71:798-805.

Deneer, J. W., C. J. Van Leeuwen, W. Seinen, J. L. Maas-Diepeveen, and J. L. M. Hermens. 1989. QSAR Study of the Toxicity of Nitrobenzene Derivatives Towards Daphnia magna, Chlorella pyrenoidosa and Photobacterium phosphoreum. Aquat Toxicol 15: 83-98.

Ek, H., G. Dave, G. Birgersson, L. Förlin. 2003. Acute Effects of 2,4,6-trinitrotoluene (TNT) on haematology parameters and hepatic EROD-activity in rainbow trout (Oncorhynchus mykiss). Aquatic Ecosystem Health and Management 6:415-421.

Ek, H., G. Dave, J. Sturve, B.C. Almroth, E. Stephensen, L. Förlin, G. Birgersson. 2005. Tentative biomarkers for 2,4,6-Trinitrotoluene (TNT) in fish (Oncorhynchus mykiss). Aquat Toxicol 72:221-230.

Ek, H., G. Dave, E. Nilsson, J. Sturve, G. Birgersson. 2006. Fate and effects of 2,4,6trinitrotoluene (TNT) from dumped ammunition in a field study with fish and invertebrates. Arch Environ Contam Toxicol 51:244-252.

Ek, H., E. Nilsson, G. Birgersson, G. Dave. 2007. TNT leakage through sediment to water and toxicity to Nitocra spinipes. Ecotoxicol Environ Saf 57:341-348.

Ek, H., E. Nilsson, G. Dave. 2008. Effects of TNT leakage from dumped ammunition on fish and invertebrates in static brackish water systems. Ecotoxicol Environ Saf 69:104-111.

Elovitz, M. S., E. J. Weber. 1999. Sediment mediated reduction of 2,4,6-trinitrotoluene and fate of the resulting aromatic (poly)amines. Environ Sci Technol 33:26172625 .

ENSR International. 2005. Derivation of toxicity reference values for the acute and chronic toxicity of hexahydro-1,3,5-trinitro- 1,3,5-triazine (RDX) to marine aquatic organisms - Final report. Prepared by Parametrix, Inc., Albany, OR, and ENSR International, Redmond, WA. ENSR Project No. 09000-279-400 - U.S. Army Contract No. DACA67-00-D-2009.

Eriksson, J., S. Frankki, A. Shchukarev, U. Skyllberg. 2004. Binding of 2,4,6trinitrotoluene, aniline, and nitrobenzene to dissolved and particulate soil organic matter. Environ Sci Technol 38:3074-3080.

Fisher, D., D. Burton, and R. Paulson. 1989. Comparative acute toxicity of diethyleneglycol dinitrate to freshwater aquatic organisms. Environ Toxicol Chem: 8: 545-550.

Fung, V., C. Teague, A. Wilson, C. Robinson, and P. Vinh. 2009. Development of a production scale process for the manufacture of IMX-101 at HSAAP. Presentation made at: 2009 Insensitive Munitions and Energetic Materials Technology Symposium, Tucson, AZ, 11-14 May 2009. 
Garcia-Reyero, N., H. C. Poynton, A. J. Kennedy, X. Guan, B. L. Escalon, B. Chang, J. Varshavsky, A. V. Loguinov, C. D. Vulpe, and E. J. Perkins. 2009. Biomarker Discovery and Transcriptomic Responses in Daphnia magna exposed to Munitions Constituents. Environ Sci Technol 43: 4188-4193.

Garcia-Reyero, N., B. L. Escalon, P. R. Loh, J. G. Laird, A. J. Kennedy, B. Berger, and E. J. Perkins. 2012. Assessment of Chemical Mixtures and Groundwater Effects on Daphnia magna Transcriptomics. Environmental Science \& Technology 46: 42-50.

Gobas, FAPC and H. A. Morrison. 2000. Bioconcentration and biomagnification in the aquatic environment. In: Boethling RS, Mackay D, editors. Handbook of property estimation methods for chemicals, environmental and health sciences. CRC Press. Boca Raton. FL. p 189-231

Gobas FAPC, W. de Wolf, L. P. Burkhard, E. Verbruggen, K. Plotzke. 2009. Revisiting bioaccumulation criteria for POPs and PBT assessments. Integr Environ Assess Manag 5:624-637.

Gobas, FAPC, J. B. Wilcockson, R. W. Russell, and G. D. Haffner. 1999. Mechanism of Biomagnification in fish under laboratory and field conditions. Environmental Science and Technology 33:133-141.

Goodfellow, W. L., Jr., D. T. Burton, W. C. Graves, L. W. Hall, Jr., K. R. Cooper. 1983. Acute toxicity of picric acid and picramic acid to rainbow trout, Salmo gairdneri, and American oyster, Crassostrea virginica. Water Resour Bull 19:641-648.

Green, A., D. Moore, A. D. Farrar. 1999. Chronic toxicity of 2,4,6-trinitrotoluene to a marine polychaete and an estuarine amphipod. Environ Toxicol Chem 18:17831790 .

Greenwald, I. 1926. On the Solubility of some Picrates and the Determination of Guanidines in Urine. Biochemical Journal 20: 665-667.

Griest, W. H., A. A. Vass, A. J. Stewart, and C. H. Ho. 1998. chemical and toxicological characterization of slurry reactor biotreatment of explosives-contaminated soils. Technical Report SFIM-AEC-ETCR-96186. Aberdeen, MD: U.S. Army Environmental Center.

Gust, K. A., M. S. Wilbanks, X. Guan, M. Pirooznia, T. Habib, L. Yoo, H. Wintz, C. D. Vulpe, and E. J. Perkins. 2011. Investigations of transcript expression in fathead minnow (Pimephales promelas) brain tissue reveal toxicological impacts of RDX exposure. Aquat Toxicol 101: 135-145.

Gust, K. A., B. A. Fouke, F. Z. Najar, T. Habib, G. R. Lotufo, E. Wisseman, A. M. Piggot, J. G. Laird, M. S. Wilbanks, A. Rawat, K. J. Indest, B. A. Roe, and E. J. Perkins. (in preparation). Coral-zooxanthellae meta-transcriptomics reveals integrated response to pollutant series.

Haley, M., R. Kuperman, and R. Checkai. 2009. Aquatic Toxicity of 3-Nitro-1,2,4Triazol-5-One. Public Report ECBC-TR-726. U.S. Army Research, Development and Engineering Command. 
Harrison, I., C. H. Vane. 2010. Attenuation of TNT in seawater microcosms. Water Sci Technol 61:2531-2538.

Heitmuller, P. T., T. A. Hollister and P. R. Parrish. 1981. Acute toxicity of 54 industrial chemicals to sheepshead minnows (Cyprinodon variegatus). Bulletin of Environmental Contamination and Toxicology 27: 596-604.

Houston, J. G., and G. R. Lotufo. 2005. Dietary exposure of fathead minnows to the explosives TNT and RDX and to the pesticide DDT using contaminated invertebrates. Int. J. Environ. Res. Public Health 2:286-292.

Jenkins, T. F., J. C. Pennington, T. A. Ranney, T. E. Berry, P. H. Miyares, M. E. Walsh. 2001. Characterization of explosives contamination at military firing ranges. ERDC TR-01-5 Final/Technical Report. Hanover, NH: Engineer Research and Development Center, U.S. Army Corps of Engineers.

Johnson, L. R., R. Davenport, H. Balbach, and D. J. Schaeffer. 1994. Phototoxicology. 3. Comparative toxicity of trinitrotoluene and aminodinitrotoluenes to Daphnia magna, Dugesia dorotocephala, and sheep erythrocytes. Ecotoxicol Environ Saf 27: 34-49.

Juhasz, A. L., and R. Naidu. 2007. Explosives: Fate, dynamics, and ecological impact in terrestrial and marine environments. Rev Environ Contam Toxicol 191:163-215.

Kaplan, D. L., J. H. Cornell, A. M. Kaplan. 1982. Decomposition of nitroguanidine. Environ Sci Technol 16: 488-492.

Kennedy, A.J., C.D. Lounds, N.L. Melby, J.G. Laird, B. Winstead, S.M. Brasfield, and M.S. Johnson. Development of Environmental Health Criteria for Insensitive Munitions: Aquatic Ecotoxicological Exposures Using 2,4-Dinitroanisole. (In preparation.) Vicksburg, MS: U.S. Army Engineer Research and Development Center.

Kuperman, G. R., M. Simini, S. Siciliano, and P. Gong. 2009. Effects of energetic materials on soil organisms. In Ecology of explosives, ed. G. I. Sunahara, G. R. Lotufo, R. G. Kuperman, and J. Hawari. Boca Raton, FL: CRC.

Lachance, B., A. Y. Renoux, M. Sarrazin, J. Hawari, and G. I. Sunahara. 2004. Toxicity and bioaccumulation of reduced TNT metabolites in the earthworm Eisenia andrei exposed to amended forest soil. Chemosphere 55:1339-1348.

Lang, P. Z., Y. Wang, D. B. Chen, N. Wang, X. M. Zhao, and Y. Z. Ding. 1997. Bioconcentration, elimination and metabolism of 2,4-dinitrotoluene in carps (Cyprinus carpio L.). Chemosphere 35:1799-1815.

LeBlanc, G. A. 1980. Acute toxicity of priority pollutants to water flea Daphnia magna. Bulletin of Environmental Contamination and Toxicology 24: 684-691.

Leung, K. H., M. Yao, R. Stearns, and S. H. Chiu. 1995. Mechanism of bioactivation and covalent binding of 2,4,6-trinitrotoluene. Chemico-Biological Interactions 97: $37-51$ 
Lewis, J., R. Martel, L. Trepanier, G. Ampleman, S. Thiboutot. 2009. Quantifying the transport of energetic materials in unsaturated sediments from cracked unexploded ordnance. J Environ Qual 38:2229-2236.

Liu, D. H., H. C. Bailey, and J. G. Pearson. 1983a. Toxicity of a Complex Munitions Wastewater to Aquatic Organism. In Bishop, WE, Cardwell, RD, Heidolph, BB, eds, Aquatic Toxicology and Hazard Assessment: Sixth Symposium, ASTM STP 802. Philadelphia, PA: American Society for Testing Materials.

Liu, D. H., R. J. Spanggord, H. C. Bailey, H. S. Javitz, and D. C. L. Jones. 1983b. Toxicity of TNT Wastewaters to Aquatic Organisms. Final Report. VoI. II. Acute Toxicity of Condensate Wastewater and 2,4-Dinitrotoluene. DSU-4262. Menlo Park, CA: SRI International.

Liu, D. H., R. J. Spanggord, H. C. Bailey, H. S. Javitz, and D. C. L. Jones. 1983c. Toxicity of TNT Wastewaters to Aquatic Organisms. Final Report. Vol. I. Acute Toxicity of LAP Wastewater and 2,4,6-Trinitrotoluene. AD-A142 144. SRI International, Menlo Park, CA.

Liu, Y. Y., A. Y. Lu, R. A. Stearns, and S. H. Chiu. 1992. In vivo covalent binding of $\left[{ }_{14} \mathrm{C}\right]$ trinitrotoluene to proteins in the rat. Chem Biol Interact. 82: 1-19.

Lotufo, G. R., J. D. Farrar, L. S. Inouye, T. S. Bridges, and D. B. Ringelberg. 2001. Toxicity of sediment-associated nitroaromatic and cyclonitramine compounds to benthic invertebrates. Environ Toxicol Chem 20:1762-1771.

Lotufo, G. R., and J. D. Farrar. 2005. Comparative and mixture sediment toxicity of trinitrotoluene and its major transformation products to a freshwater midge. Arch Environ Contam Toxicol 49:333-342.

Lotufo, G. R., and M. J. Lydy. 2005. Comparative toxicokinetics of explosive compounds in sheepshead minnows. Arch Environ Contam Toxicol 49:206-214.

Lotufo, G. R., M. Nipper, R. S. Carr, and J. M. Conder. 2009a. Fate and Toxicity of Explosives in Sediment. In Sunahara, GI, Lotufo, GR, Kuperman, RG, Hawari, J, eds, Ecotoxicology of Explosives. CRC Press, Boca Raton, FL, pp. 117-134.

Lotufo, G. R., M. J. Lydy, G. L. Rorrer, O. Cruz-Uribe, and D. P. Cheney. 2009b. Bioconcentration, Bioaccumulation and Biotransformation of Explosives and Related Compounds in Aquatic Organisms. In Sunahara, GI, Lotufo, GR, Kuperman, RG, Hawari, J, eds, Ecotoxicology of Explosives. Boca Raton, FL: CRC Press.

Lotufo, G. R., and W. Blackburn. 2010. Bioaccumulation of TNT and DDT in sheepshead minnows, Cyprinodon variegatus L., following feeding of contaminated invertebrates. Bulletin of Environmental Contamination and Toxicology 84:545549 .

Lotufo, G. R., W. Blackburn, and A. B. Gibson. 2010a. Toxicity of trinitrotoluene to sheepshead minnows in water exposures. Ecotoxicol Environ Saf 73:718-726.

Lotufo, G. R., A. B. Gibson, and Y. J. Leslie. 2010b. Toxicity and bioconcentration evaluation of RDX and HMX using sheepshead minnows in water exposures. Ecotoxicol Environ Saf 73:1653-1657. 
Lotufo, G. R., W. Blackburn, S. J. Marlborough, and J. W. Fleeger. 2010c. Toxicity and bioaccumulation of TNT in marine fish in sediment exposures. Ecotoxicol Environ Saf 73:1720-1727.

Lotufo, G. R. 2011. Whole-body and body-part-specific bioconcentration of explosive compounds in sheepshead minnows. Ecotoxicol Environ Saf 74:301-306.

MacDonald, J. A., M. J. Small, and M. G. Morgan. 2009. Quantifying the risks of unexploded ordnance at closed military bases. Environ Sci Technol 43:259-265.

McCarty, L. S., and D. Mackay. 1993. Enhancing ecotoxicological modeling and assessment. 420 Environ Sci Technol 27: 1719 - 1728

McDonald, J. 2008. Final Report - UXO Detection and Characterization in the Marine Environment. ESTCP Project MM-0324. Washington, DC.

Meador, J. 2006. Rationale and procedures for using the tissue-residue approach for toxicity assessment and determination of tissue, water and sediment quality guidelines for aquatic organisms. HumEcol Risk Assess 12:1018 ${ }^{+} 1073$.

Meylan, W. M., P. H. Howard, R. S. Boethling, D. Aronson, H. Printup, and S. Gouchie. 1999. Improved method for estimating bioconcentration/bioaccumulation factor from octanol/water partition coefficient. Environ Toxicol Chem 18:664-672.

Monteil-Rivera, F., A. Halasz, C. Groom, J. S. Zhao, S. Thiboutot, G. Ampleman, and J. Hawari. 2009. Fate and transport of explosives in the environment. In Sunahara, GI, Lotufo, GR, Kuperman, RG, Hawari, J, eds, Ecotoxicology of Explosives. Boca Raton, FL: CRC Press.

Mukhi, S., X. P. Pan, G. P. Cobb, and R. Patino. 2005. Toxicity of hexahydro-1,3,5trinitro-1,3,5-triazine to larval zebrafish (Danio rerio). Chemosphere 61:178-185.

Mukhi, S., and R. Patino. 2008. Effects of hexahydro-1,3,5-trinitro-1,3,5-triazine (RDX) in zebrafish: General and reproductive toxicity. Chemosphere 72:726-732.

Nipper, M., R. S. Carr, J. M. Biedenbach, R. L. Hooten, K. Miller, and S. Saepoff. 2001. Development of marine toxicity data for ordnance compounds. Arch Environ Contam Toxicol 41:308-318.

Nipper, M., R. S. Carr, J. M. Biedenbach, R. L. Hooten, and K. Miller. 2002. Toxicological and chemical assessment of ordnance compounds in marine sediments and porewaters. Mar Pollut Bull 44:789-806.

Nipper, M., Y. Qian, R. S. Carr, and K. Miller. 2004. Degradation of picric acid and 2,6DNT in marine sediments and waters: the role of microbial acitivity and ultraviolet exposure. Chemosphere 56:519-530.

Nipper, M., R. S. Carr, J. M. Biedenbach, R. L. Hooten, and K. Miller. 2005. Fate and effects of picric acid and 2,6-DNT in marine environments: Toxicity of degradation products. Mar Pollut Bull 50:1205-1217.

Nipper, M., R. S. Carr, and G. R. Lotufo. 2009. Aquatic toxicology of explosives. In Sunahara, GI, Lotufo, GR, Kuperman, RG, Hawari, J, eds, Ecotoxicology of Explosives. CRC Press, Boca Raton, FL, pp. 77-115. 
Noblis. 2011. Physicochemical Properties for Explosives at the Shallow Water Site Hawaii 6 (HI-06), O'ahu, Hawaii. Prepared for National Oceanic and Atmospheric Administration under contract GS10F0189T. Falls Church, Virginia.

O'Sullivan, D. W., J. R. Denzel, Prak DJ Luning. 2011. Photolysis of 2,4,6-trinitrotoluene in seawater: effect of salinity and nitrate concentration. In Chappell MA, Price CL, George RD, editors. Environmental Chemistry of Explosives and Propellant Compounds in Soils and Marine Systems: Distributed Source Characterization and Remedial Technologies. ACS Symposium Series. American Chemical Society.

Ownby D. R., J. B. Belden, G. R. Lotufo, and M. J. Lydy. 2005. Accumulation of trinitrotoluene (TNT) in aquatic organisms: Part 1 - Bioconcentration and distribution in channel catfish (Ictalurus punctatus). Chemosphere 58:1153-1159.

Paden, N. E., E. E. Smith, J. D. Maul, and R. J. Kendall. 2011. Effects of chronic 2,4,6,trinitrotoluene, 2,4-dinitrotoluene, and 2,6-dinitrotoluene exposure on developing bullfrog (Rana catesbeiana) tadpoles. Ecotoxicol Environ Saf 74: 924928.

Pascoe, G. A., K. Kroeger, D. Leisle, and R. J. Feldpausch. 2010. Munition constituents: Preliminary sediment screening criteria for the protection of marine benthic invertebrates. Chemosphere 81:807-816.

Pearson, J. G., J. P. Glennon, J. J. Barkley, and J. W. Highfill. 1979. An approach to the toxicological evaluation of a complex industrial wastewater, pp. 284-301. In L.L.Marking and R.A.Kimerle, editors, Aquatic Toxicology and Hazard Assessment: Sixth Symposium . American Society for Testing Materials, Philadelphia, PA.

Pennington, J. C., and J. M. Brannon. 2002. Environmental fate of explosives. Thermochimica Acta 384:163-172.

Pennington, J. C., C. A. Hayes, S. Yost, T. A. Crutcher, T. E. Berry, J. U. Clarke, and M. J. Bishop. 2008. Explosive residues from blow-in-place detonations of artillery munitions. Soil Sed Contam 17:163-180.

Pennington, J. C., G. R. Lotufo, C. A. Hayes, B. Porter, and R. D. George. 2011. TNT, RDX, and HMX association with organic fractions of marine sediments and bioavailability implications. In Chappell MA, Price CL, George RD, editors. Environmental Chemistry of Explosives and Propellant Compounds in Soils and Marine Systems: Distributed Source Characterization and Remedial Technologies. ACS Symposium Series. American Chemical Society.

Peters, G. T., D. T. Burton, R. L Paulson, and S. D. Turley. 1991. The acute and chronic toxicity of hexahydro-1,3,5-trinitro-1,3,5-traizine (RDX) to three freshwater invertebrates. Environ Toxicol Chem 10:1073-1081.

Podlipna, R., Z. Fialova, and T. Vanek. 2008. Toxic effect of nitroesters on plant tissue cultures. Plant Cell Tiss Org 94:305-311.

Reddy, G., T. V. Reddy, H. Choudhury, F. B. Daniel, and G. J. Leach. 1997. Assessment of environmental hazards of 1,3,5-trinitrobenzene. Journal of Toxicol Envi Health 52:447-460. 
Redman, A. D., E. Mihaich, K. Woodburn, P. Paquin, D. Powell, J. A. McGrath, and D. M. Di Toro. 2012. Tissue-based risk assessment of cyclic volatile methyl siloxanes. Environ Toxicol Chem 31: 1911-1919.

Rocheleau, S., R. G. Kuperman, S. G. Dodard, M. Sarrazin, K. Savard, L. Paquet, J. Hawari, R. T. Checkai, S. Thiboutot, G. Ampleman, and G. I. Sunahara. 2011. Phytotoxicity and uptake of nitroglycerin in a natural sandy loam soil. Sci Total Environ 40:5284-5291.

Rosen, G, and G. R. Lotufo. 2005. Toxicity and fate of two munitions constituents in spiked sediment exposures with the marine amphipod Eohaustorius estuarius. Environ Toxicol Chem 24:2887-2897.

Rosen, G, and G. R. Lotufo. 2007a. Toxicity of explosive compounds to the marine mussel, Mytilus galloprovincialis, in aqueous exposures. Ecotoxicol Environ Saf 68:228-236.

Rosen, G., and G. R. Lotufo. 2007b. Bioaccumulation of explosive compounds in the marine mussel, Mytilus galloprovincialis. Ecotoxicol Environ Saf 68:237-245.

Rosen, G., and G. R. Lotufo. 2010. Fate and effects of Composition B in multispecies marine exposures. Environ Toxicol Chem 29:1330-1337.

Rosenblatt, D. H., E. P. Burrows, W. R. Mitchell, and D. L. Parmer. 1991. Organic explosives and related compounds. In: Hutzinger O (ed) The Handbook of Environmental Chemistry, vol 3. part G. Springer-Verlag, New York, 195-234.

S\&ME. 2007. Biomonitoring retest report BAE Systems, Kingsport, TN. S\&ME Project No. 1434-07-537. Report to BAE Systems, 22 October 2007. Kingsport, TN.

Saka, M. 2004. Developmental toxicity of p,p '-dichlorodiphenyltrichloroethane, 2,4,6trinitrotoluene, their metabolites, and benzo[a]pyrene in Xenopus laevis embryos. Environ Toxicol Chem 23: 1065-1073.

Savard, K., M. Sarrazin, S. G. Dodard, F. Monteil-Rivera, R. G. Kuperman, J. Hawari, and G. I. Sunahara. 2010. Role of soil Interstitial water in the accumulation of hexahydro-1,3,5-trinitro-1,3,5-triazine in the earthworm Eisenia Andrei. Environ Toxicol Chem 29:998-1005.

Sayers, L. E. 2009a. Nitrotriazolone (NTO, CAS No. 932-64-9) - Acute toxicity to water fleas (Daphnia magna) under flow-through conditions, following OPPTS Draft Guideline 850.1010. Springborn Smithers Study No. 13949.6107, Report to BAE Systems. 1 December 2009.

Sayers, L. E. 2009b. Nitrotriazolone (NTO, CAS No. 932-64-9) - Acute Toxicity to Rainbow Trout (Oncorhynchus mykiss) under flow-through conditions, following OPPTS Draft Guidance 850.1075. Springborn Smithers Study No. 13949.6108, Report to BAE Systems. 2 November 2009.

SERDP (Strategic Environmental Research and Development Program) 2010. White Paper, Munitions in the Underwater Environment: State of the Science and Knowledge Gaps. June. 12pp. 
Serrano, R., F. Hernandez, J. B. Pena, V. Dosda, and J. Canales. 1995. Toxicity and bioconcentration of selected organophosphorus pesticides in Mytilus galloprovincialis. Arch Environ Contam Toxicol 29:284-290.

Sims J. G., and J. A. Steevens. 2008. The role of metabolism in the toxicity of 2,4,6trinitrotoluene and its degradation products to the aquatic amphipod Hyalella azteca. Ecotoxicol Environ Saf 70:38-46.

Singh, N., A. E. Berns, D. Hennecke, J. Hoerner, W. Koerdel, and A. Schaeffer 2010. Effect of soil organic matter chemistry on sorption of trinitrotoluene and 2,4dinitrotoluene. $J$ Hazard Mater 173:343-348.

Singh, B, J. Kaur, and K. Singh. 2012. Microbial remediation of explosive waste. Critical Reviews in Microbiology 38: 152-167.

Snell, T. W., and B. D. Moffat. 1992. A 2-d life cycle test with the totifer Brachionus calyciflorus. Environ Toxicol Chem 11:1249-1257.

Spanggord, R. J., T-W Chou, T. M. Mill, R. T. Podoll, J. C. Harper, and D. S. Tse. 1985. Environmental fate of nitroguanidine, diethyleneglycol dinitrate, and hexachloroethane smoke- Phase I. Draft Final Report. DAMD17-84-C-4252. Menlo Park, CA: SRI International.

Steevens, J. A., B. M. Duke, G. R. Lotufo, and T. S. Bridges. 2002. Toxicity of the explosives 2,4,6-trinitrotoluene, hexahydro-1,3,5-trinitro-1,3,5-triazine, and octahydro-1,3,5,7-tetranitro-1,3,5,7-tetrazocine in sediments to Chironomus tentans and Hyalella azteca: Low-dose hormesis and high-dose mortality. Environ Toxicol Chem 21:1475-1482.

Talmage, S. S., D. M. Opresko, C. J. Maxwell, C. J. Welsh, F. M. Cretella, P. H. Reno, and F. B. Daniel. 1999. Nitroaromatic munition compounds: Dnvironmental effects and screening values. Rev Environ Contam Toxicol 161:1-156.

Thorn, K. A., P. G. Thorne, and L. G. Cox. 2004. Alkaline hydrolysis/polymerization of 2,4,6-trinitrotoluene: Characterization of products by $\mathrm{C}-13$ and N-15 NMR. Environ Sci Technol 38:2224-2231.

Thorne, P. G., and T. F. Jenkins. 1997. A field method for quantifying ammonium picrate and picric acid in soil. Field Anal. Chem. Technol. 1: 165-179.

Toussaint, M. W., T. R. Shedd, W. H. van der Schalie, and G. R. Leather GR. 1995. A Comparison of Standard Acute Toxicity Tests with Rapid-Screening Toxicity Tests. Environ Toxicol Chem 14: 907-915.

U.S. Army. 1961. Encyclopedia of Explosives and Related Items. United States Army Research and Development Command TACOM, ARDEC, Warheads, Energetics and Combat Support Center, Picatinny Arsenal, NJ.

Headquarters, U.S. Department of the Army. 1990. Military explosives. Department of the Army, Headquarters, Washington, DC, Army Technical Manual TM 9-1300214 (with Change No. 4). 
Headquarters, U.S. Department of the Army. 1993. Ammunition, general. Department of the Army, Headquarters, Washington, DC, Army Technical Manual TM 9-1300200 (with Change No. 5).

U.S. Army Center for Health Promotion and Preventive Medicine (USACHPPM). 2001. Wildlife Toxicity Assessment for Nitroglycerin. Project Number 39-EJ-1138-01F. Aberdeen Proving Ground, Maryland.

U.S. Army Public Health Command (USAPHC). 2011. Wildlife Toxicity Assessment for 2,4 \& 2,6 Dinitrotoluenes. PHN No. 0512-1.

U.S. Navy. 1987. Technical manual for use of explosives in underwater salvage. Washington, DC: Naval Sea Systems Command.. NAVSEA-SWo61-AA-MMA010.

U.S. Navy. 1999. Chapter 3: Characteristics of explosives and ammunition. OP5 Volume 1 Sixth Revision. Naval Sea Systems Command, Department of the Navy, Washington, D.C.

U.S. EPA. 1993. Handbook approaches for the remediation of federal facility sites contaminated with explosive of radioactive wastes. Washington, DC: Office of Research and Development, United States Environmental Protection Agency. $\mathrm{EPA} / 625 / \mathrm{R}-93 / 013$.

U.S.EPA. 2012. Site Characterization for munitions constituents: EPA federal facilities forum issue paper. Solid Waste and EPA-505-S-11-001. Emergency Response, Washington, DC: United States Environmental Protection Agency.

van der Schalie, W. H. 1985. The Toxicity of Nitroguanidine and Photolyzed Nitroguanidine to Freshwater Aquatic Organisms. AD-A153 045 (8404). Fredrick, MD: US Army Medical Bioengineering Research and Development Laboratory (Fort Detrick).

van Ham, N., A. Creemers, and D. Meuken. 2007. Risks of Sea Dumped Ammunition. Paper presented at "Security Impact of Munition and Propellant Disposal," Bulgaria, 12-14 September 2007.

Wang, P. F., Q. Liao, R. George, and W. Wild, 2011. Release Rate and Transport of Munitions Constituents from Breached Shells in Marine Environment. Environmental Chemistry of Explosives and Propellant Compounds in Soils and Marine Systems: Distributed Source Characterization and Remedial Technologies. American Chemical Society, pp. 317-340.

Wang, Y, Z. J. Wang, C. X. Wang, and W. H. Wang. 1999. Uptake of weakly hydrophobic nitroaromatics from water by semipermeable membrane devices (SPMDs) and by goldfish (Carassius auratus). Chemosphere 38:51-66.

Warner, C.M., K.A. Gust, J.K. Stanley, T. Habib, M. S. Wilbanks, N. Garcia-Reyero, and E. J. Perkins EJ . 2012. A systems toxicology approach to elucidate the mechanisms involved in RDX species-specific sensitivity. Environ. Sci. Technol. 46: 77907798 . 
Won, W. D., L. H. DiSalvo, and J. Ng. 1976. Toxicity and mutagenicity of 2,4,6trinitrotoluene and its microbial metabolites. Appl Environ Microbiol 31:576580 .

Xu, J. B., and N. Jing. 2012. Effects of 2,4-dinitrotoluene exposure on enzyme activity, energy reserves and condition factors in common carp (Cyprinus carpio). $J$ Hazard Mater 203: 299-307.

Yoo, L. J., G. R. Lotufo, A. B. Gibson, J. A. Steevens, and J. G. Sims. 2006. Toxicity and bioaccumulation of 2,4,6-trinitrotoluene in fathead minnow (Pimephales promelas). Environ Toxicol Chem 25:3253-3260.

Zhao, Y-Y, X. Yuan, G-D. Ji, and L-X Sheng. 1997. Quantitative structure-acitivity relationships of nitroaromatic compounds to four aquatic organisms.

Chemosphere 34:1837-1844. 


\section{Appendix A: Acronyms and Abreviations}

\begin{tabular}{|c|c|}
\hline Am-DNT & Aminodinitrotoluene \\
\hline $\mathrm{AP}$ & Ammonium perchlorate (Explosive D) \\
\hline $\mathrm{BCF}$ & Bioconcentration Factor \\
\hline BIP & Blow-in-Place \\
\hline BRAC & Base Realignment and Closure \\
\hline CBR & Critical Body Residue \\
\hline DMM & Discarded Military Munitions \\
\hline DNB & Dintrobenzene \\
\hline DoD & Department of Defense \\
\hline DANT & Diaminonitrotoluene \\
\hline DEGDN & Diethylene glycol dinitrate \\
\hline DNT & Dinitrotoluene \\
\hline EGDN & Ethyleneglycoldinitrate \\
\hline FUDS & Formally Used Defense Sites \\
\hline HMX & $\begin{array}{l}\text { High Melting Explosive (or octahydro-1,3,5,7- } \\
\text { tetranitro-1,3,5,7-tetrazocine) }\end{array}$ \\
\hline LD50 & Lethal dose 50 \\
\hline $\log \mathrm{K}_{\mathrm{OC}}$ & Organic carbon soil partition coefficient \\
\hline $\log \mathrm{K}_{\mathrm{OW}}$ & Octanol/water partition coefficient \\
\hline
\end{tabular}




$\begin{array}{ll}\text { MC } & \text { Munitions Constituents } \\ \text { MEC } & \text { Munitions and explosives of concern } \\ \text { NC } & \text { Nitrocellulose } \\ \text { NG } & \text { Nitroglycerine } \\ \text { NQ } & \text { Nitroguanidine } \\ \text { PEP } & \text { propellants, explosives, or pyrotechnics } \\ \text { PBX } & \text { Plastic or Polymer Bonded Exposives } \\ \text { PETN } & \text { Pentaerythrite tetranitrate } \\ \text { RDX } & \text { Royal Demolition Explosive (Cyclo-1,3,5- } \\ & \begin{array}{l}\text { trimethylene-2,4,6-trinitramine, hexahydro-1,3,5- } \\ \text { trinitro-1,3,5-triazine or cyclonite) }\end{array} \\ & \text { 1,3,5-trinitrobenzenes } \\ 1,3,5-T N B & 2,4,6-\text { Trinitrotoluene } \\ \text { TNT } & \text { US Army Corps of Engineers } \\ \text { USACE } & \text { US Environmental Protection Agency } \\ \text { USEPA } & \text { Unexploded Ordnance } \\ \text { UXO } & \end{array}$




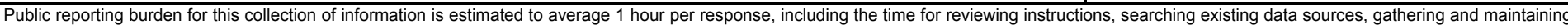

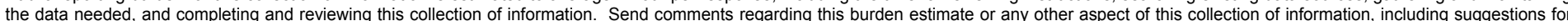

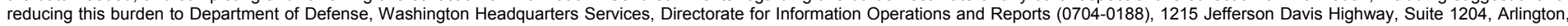

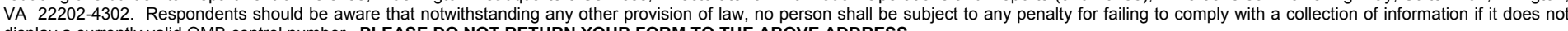
display a currently valid OMB control number. PLEASE DO NOT RETURN YOUR FORM TO THE ABOVE ADDRESS.
1. REPORT DATE (DD-MM-YYYY) 2. REPORT TYPE
June 2013 Final report
3. DATES COVERED (From - To)

\section{TITLE AND SUBTITLE}

Summary Review of the Aquatic Toxicology of Munitions Constituents

5a. CONTRACT NUMBER

5b. GRANT NUMBER

5c. PROGRAM ELEMENT NUMBER

\section{AUTHOR(S)}

\section{5d. PROJECT NUMBER}

Guilherme R. Lotufo, Gunther Rosen, William Wild, Geoffrey Carton

5e. TASK NUMBER

5f. WORK UNIT NUMBER

\section{PERFORMING ORGANIZATION NAME(S) AND ADDRESS(ES)}

\section{PERFORMING ORGANIZATION REPORT} NUMBER

U.S. Army Engineer Research and Development Center

Environmental Laboratory

3909 Halls Ferry Road, Vicksburg, MS 39180-6199

\section{SPONSORING / MONITORING AGENCY NAME(S) AND ADDRESS(ES)}

ERDC/EL TR-13-8

Office of the Deputy Assistant Secretary of the Army for Environment, Safety and Occupational Health (DASA-ESOH)

110 Army Pentagon Room 3E464

Washington, D.C. 20310-0110

\section{DISTRIBUTION / AVAILABILITY STATEMENT}

Approved for public release; distribution unlimited.

\section{SUPPLEMENTARY NOTES}

\section{ABSTRACT}

Military munitions are present in waters around the world, including those waters located at current and former Department of Defense sites. This report provides a review of the aquatic ecotoxicology of munitions constituents (MC), including nitroaromatics (2,4,6trinitrotoluene (TNT), dinitrotoluenes (DNTs), 1,3,5-trinitrobenzene (TNB), 2,4,6-trinitrophenylmethylnitramine (tetryl) and 2,4,6trinitrophenol (picric acid)); nitrate esters (nitrocellulose (NC), pentaerythritoltetranitrate (PETN), nitroglycerine (NG)); and nitramines (hexahydro-1,3,5- trinitro-1,3,5-triazine (RDX), and octahydro-1,3,5,7-tetranitro-1,3,5,7-tetrazocine (HMX)). The major focus of this report is on the fate and effects of MC in the marine environment. Most MC observed in this study rapidly degraded in aqueous exposure systems and nitroaromatics showed a significant binding affinity with organic matter. To support the assessment of risk from MC in aquatic environments, laboratory-based toxicity data have been derived for a variety of aquatic species for both lethal and sublethal exposure endpoints using spiked water or sediment. Frequently, unrealistically high concentrations were used to derive toxicity benchmarks. In general, nitramines were less toxic than nitroaromatics, with a wide range of sensitivity among species. MC are weakly hydrophobic and bioaccumulative potential was low, as expected. High elimination rates for MC resulted in a virtually complete loss of body residue within hours to days following transfer to clean water. Uptake of TNT resulted in the substantial formation of bound residues. For fish, aqueous exposure was the dominant route of exposure to explosive compounds, with dietary uptake providing only minimal contribution. More realistic exposures using Composition B and multiple species found the presence of munitions in aquatic environments unlikely to result in biological effects. Verification of this conclusion should be pursued by determining site-specific exposure risk.
15. SUBJECT TERMS
Explosives
Toxicity
Bioaccumulation
Munitions constituents
Unexploded ordnance

Composition B

Sediment

16. SECURITY CLASSIFICATION OF:

\section{a. REPORT}

UNCLASSIFIED

b. ABSTRACT
UNCLASSIFIED

c. THIS PAGE

UNCLASSIFIED
17. LIMITATION 17. LIMITATION
OF ABSTRACT

18. 18. NUMBER OF PAGES

122 19a. NAME OF RESPONSIBLE PERSON

Guilherme R. Lotufo 19b. TELEPHONE NUMBER (include area code) 6018317310 